\title{
IntechOpen
}

\section{Non-tubal Ectopic Pregnancy}

Edited by Julio Elito Jr. 



\section{Non-tubal Ectopic Pregnancy}

Edited by Julio Elito Jr. 

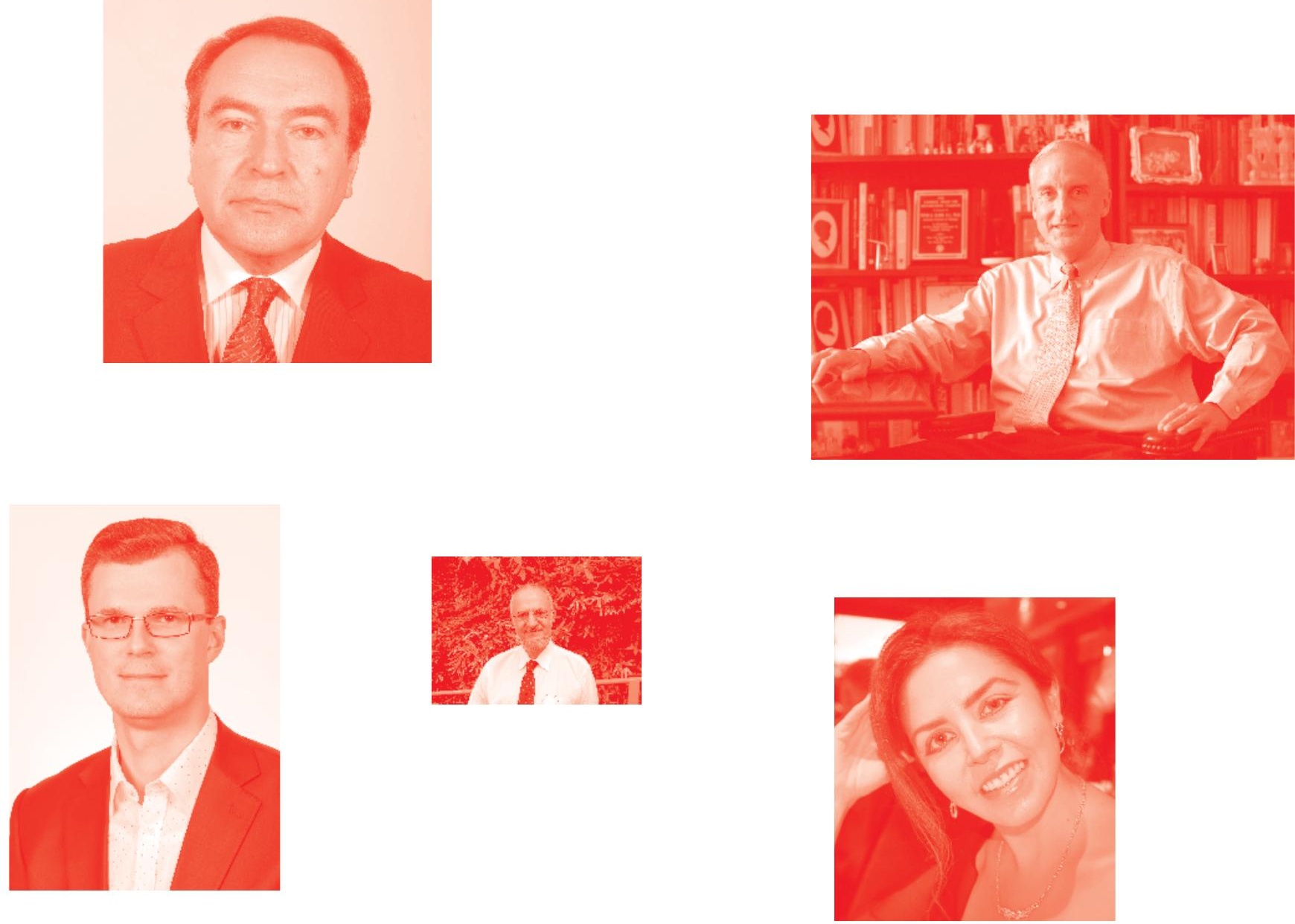

Supporting open minds since 2005
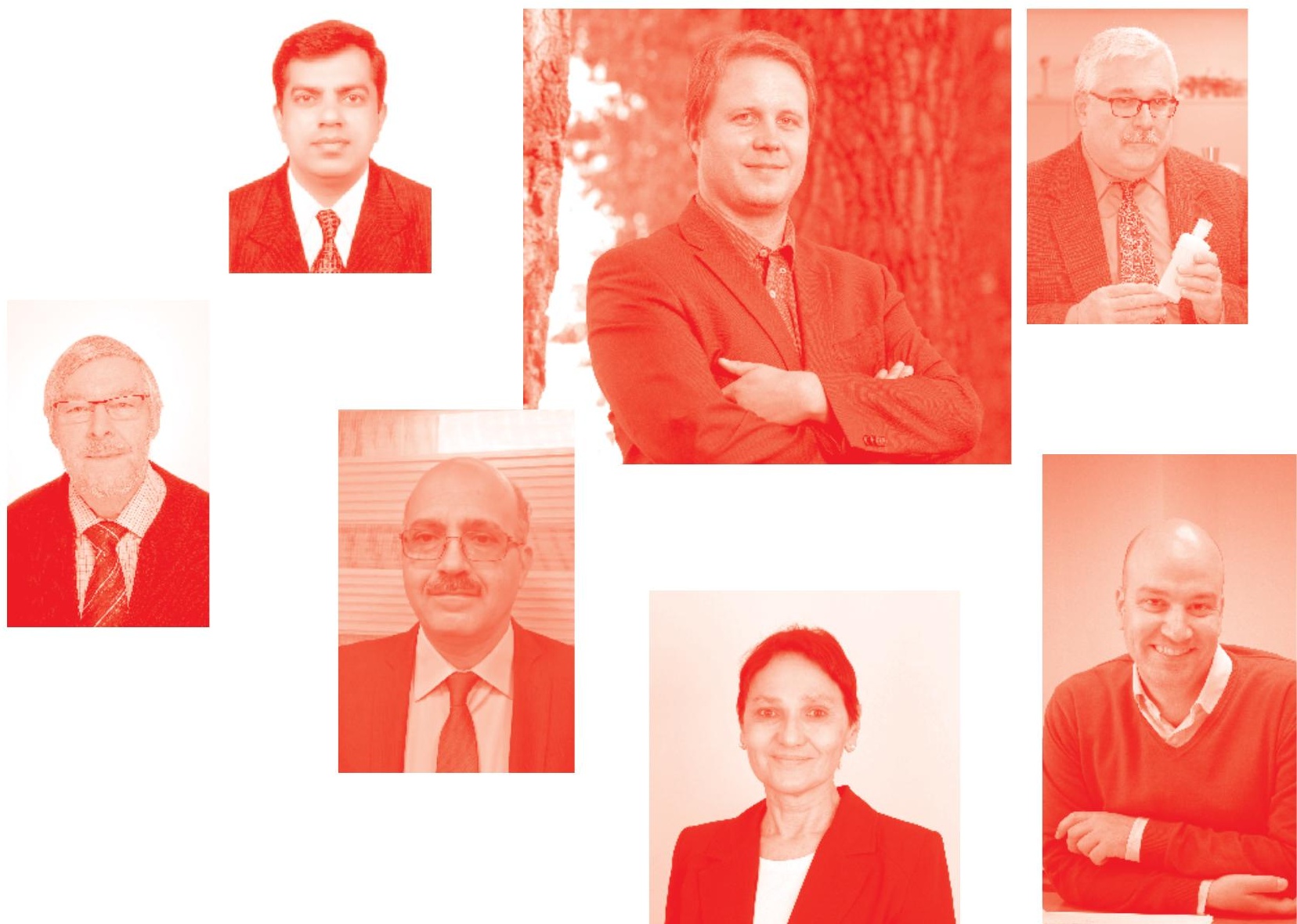
Non-tubal Ectopic Pregnancy

http : //dx. doi.org/10.5772/intechopen. 78860

Edited by Julio Elito Jr.

Contributors

Dionysios Karavyrakis, Stelios Fiorentzis, Theodoros Margetousakis, Chrysostomos Georgellis, Pantelis Kotridis, Dimitra Oikonomopoulou, Alexandros Karamperis, Georgia Sotiropoulou Karamperis, Workineh Tadesse, Ozer Birge, Aliye Nigar Serin, Rubens Musiello, Eduardo Souza, Jair Fava, Luiz Camano, Taro Koshiishi, Satoru Takeda, Jun Takeda, Takashi Yorifuji, Christopher A Enakpene, Adebayo Adeniyi, Julio Elito Jr.

( ) The Editor(s) and the Author(s) 2020

The rights of the editor(s) and the author(s) have been asserted in accordance with the Copyright, Designs and Patents Act 1988. All rights to the book as a whole are reserved by INTECHOPEN LIMITED. The book as a whole (compilation) cannot be reproduced, distributed or used for commercial or non-commercial purposes without INTECHOPEN LIMITED's written permission. Enquiries concerning the use of the book should be directed to INTECHOPEN LIMITED rights and permissions department (permissions@intechopen.com).

Violations are liable to prosecution under the governing Copyright Law .

\section{(cc) BY}

Individual chapters of this publication are distributed under the terms of the Creative Commons Attribution 3.๑ Unported License which permits commercial use, distribution and reproduction of the individual chapters, provided the original author(s) and source publication are appropriately acknowledged. If so indicated, certain images may not be included under the Creative Commons license. In such cases users will need to obtain permission from the license holder to reproduce the material. More details and guidelines concerning content reuse and adaptation can be found at http : //www . intechopen . com/copyright-policy . html .

\section{Notice}

Statements and opinions expressed in the chapters are these of the individual contributors and not necessarily those of the editors or publisher. No responsibility is accepted for the accuracy of information contained in the published chapters. The publisher assumes no responsibility for any damage or injury to persons or property arising out of the use of any materials, instructions, methods or ideas contained in the book.

First published in London, United Kingdom, 2020 by IntechOpen IntechOpen is the global imprint of INTECHOPEN LIMITED, registered in England and Wales, registration number: 11086078 , 7th floor, 10 Lower Thames Street, London,

EC3R 6AF, United Kingdom

Printed in Croatia

British Library Cataloguing-in-Publication Data

A catalogue record for this book is available from the British Library

Additional hard and PDF copies can be obtained from orders@intechopen.com

Non-tubal Ectopic Pregnancy

Edited by Julio Elito Jr.

p. $\mathrm{cm}$.

Print ISBN 978-1-83880-454-1

Online ISBN 978-1-83880-455-8

eBook (PDF) ISBN 978-1-78984-572-3 


\section{We are IntechOpen, \\ the world's leading publisher of Open Access books}

Built by scientists, for scientists

\section{$4,700+$}

Open access books available

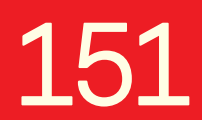

Countries delivered to

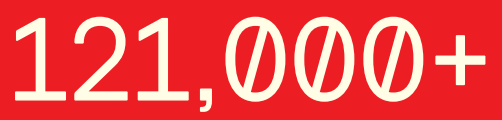

International authors and editors

Our authors are among the

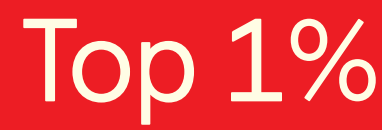

most cited scientists

Contributors from top 500 universities
40010

Downloads

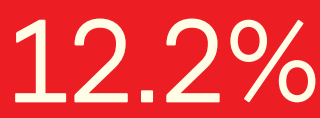

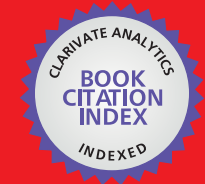

WEB OF SCIENCE ${ }^{\text {MM }}$

Selection of our books indexed in the Book Citation Index in Web of Science ${ }^{\mathrm{TM}}$ Core Collection (BKCI)

Interested in publishing with us?

Contact book.department@intechopen.com

Numbers displayed above are based on latest data collected.

For more information visit www.intechopen.com

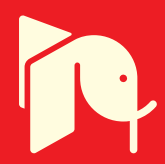





\section{Meet the editor}

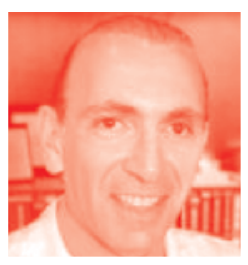

Julio Elito Junior, PhD., graduated in Medicine from Federal University of São Paulo (UNIFESP) - Brazil (1989). He obtained his Master in 1995 and his Doctorate in 1997 both from UNIFESP. He is a specialist in laparoscopy and reproductive medicine. Due to the relevance of his research, he obtained the title of Associate Professor of the Department of Obstetrics of UNIFESP (2006). He has published several articles about medical treatment in ectopic pregnancy. He has published articles on non-tubal ectopic pregnancy (cervical pregnancy, cesarean scar pregnancy, and intraligamentary abdominal pregnancy). He has published several articles in international journals. He is the reviewer in several international journals and has presented at a number of national and international congresses. He wrote the book "Ectopic Pregnancy" (2010), as well as numerous chapters in national and international books. He is editor of the book "Multiple Pregnancy: New Challenges" published by IntechOpen (2019). He has been nominated several times as Honored Professor by his students at the Medical School of Federal University of São Paulo (UNIFESP). 



\section{Contents}

Preface

Section 1

Introduction

Chapter 1

Introductory Chapter: An Overview of the Diagnosis and Treatment of Non-tubal Ectopic Pregnancy

by Julio Elito Jr

Section 2

Epidemiology

Chapter 2

Non-tubal Ectopic Pregnancy: Incidence and Diagnosis

by Aliye Nigar Serin and Özer Birge

Section 3

Diagnosis and Management

Chapter 3

Non-tubal Ectopic Pregnancy: Diagnosis and Management

by Adebayo A. Adeniyi and Christopher A. Enakpene

Chapter 4

Cervical Pregnancy Diagnosis and Management

by Rubens Bermudes Musiello, Jair Luiz Fava, Luiz Camano

and Eduardo de Souza

Chapter 5

Caesarean Scar Ectopic Pregnancy

by Workineh Getaneh Tadesse

Chapter 6

A Minimally Invasive Hemostatic Strategy for Cesarean Scar Pregnancy and Cervical Pregnancy

by Satoru Takeda, Jun Takeda, Takashi Yorifuji and Taro Koshiishi 
Chapter 7

Broad Ligament Pregnancy

by Stelios Fiorentzis, Theodoros Margetousakis, Chrysostomos Georgellis, Pantelis Kotridis, Dimitra Oikonomopoulou, Alexandros Karamperis,

Georgia Karamperi-Sotiropoulou and Dionysios Karavyrakis 


\section{Preface}

The idea of this book came about due to the increasing number of case reports in the medical literature on non-tubal ectopic pregnancies, which is mainly due to the increased number of cesarean sections and assisted reproduction. There is no consensus in the literature about the best treatment, therefore non-tubal ectopic pregnancy is still a challenge for obstetricians. Innumerous innovations in the diagnosis and management of non-tubal ectopic pregnancies have occurred in the recent years and this book offers an immersion on the subject. Throughout the book the reader is invited to visit the most important issues related to non-tubal ectopic pregnancies.

Non-tubal ectopic pregnancies presents serious complications, which are greater than in tubal pregnancies. Therefore, the maternal morbidity and mortality are higher. Due to this, an early diagnosis is crucial for the best result of the treatment. In this book we present the challenges and pitfalls for diagnosis.

In the various chapters, the authors contemplate the management of different types of non-tubal ectopic pregnancies such as cervical, interstitial, cesarean scar, ovarian, and abdominal pregnancy to which several modalities of treatment are presented: surgery, local treatment with methotrexate, systemic treatment with MTX, embolization, and specific treatments for the different sites of non-tubal pregnancy.

The book presents the most relevant aspects of the non-tubal ectopic pregnancy scenario. Authors from all over the world who have contributed to this book are experts in their areas and offer readers the best from their research.

Our intention is for the reader to be presented with the latest updates on non-tubal ectopic pregnancies.

I would like to thank my wife, Camila, and our children, João and Pedro, for their support, understanding, and love during the journey to complete this mission that will help women with non-tubal ectopic pregnancy all over the world.

Julio Elito Jr.

Associate Professor of the Department of Obstetrics at Federal University of São Paulo, São Paulo, Brazil 

Section 1

\section{Introduction}





\title{
Introductory Chapter: An Overview of the Diagnosis and Treatment of Non-tubal Ectopic Pregnancy
}

\author{
Julio Elito Jr
}

\section{Introduction}

Non-tubal ectopic pregnancies represent $7-10 \%$ of all ectopic pregnancies [1]. The incidence is increasing in the past years especially because of assisted reproduction treatment, particularly IVF, and the high rates of cesarean sections [2].

Non-tubal locations of ectopic pregnancies are cervix, cesarean section scar, interstitial portion of the fallopian tube, cornual, ovary, abdominal cavity, and broad ligament (Figure 1).

There is a broad spectrum of clinical presentation according to the location of the pregnancy. The first symptoms are pelvic pain and vaginal bleeding. In ruptured ectopic pregnancies, the patients present with severe abdominal pain, shoulder tip pain, nausea, vomiting and dizziness and collapse. However, some women with ectopic pregnancy are asymptomatic.

The diagnosis is usually late, because these pregnancies present themselves later than tubal pregnancies. Therefore, the risks of maternal morbidity and mortality are higher than in tubal ectopic pregnancies.

The management of non-tubal ectopic pregnancy depends on their location. The cases of abdominal pregnancy must be individualized and the treatment is different from the other sites of ectopic pregnancy. The diagnosis of ovarian pregnancy is usually confirmed only during the surgery.

The other sites (cesarean scar, cervical and intersticial pregnancy) follow a similar non-surgical protocol.

The classic treatment is surgery; however, it is very aggressive, because in the majority of the cases it is necessary to perform a hysterectomy. Several minimally invasive techniques have been proposed to avoid the mutilation of the uterus. Some of the alternative treatments to avoid surgery are local injection of MTX guided by transvaginal ultrasound, systemic medical treatment with methotrexate (MTX), and embolization of uterine arteries. There are several case reports in this subject but very few original articles. And in the few articles, there is no consensus on the best treatment. This book aims to provide the reader with a concise, comprehensive, and updated review of the epidemiology, diagnosis, and treatment of non-tubal ectopic pregnancy. As there is a lack of consensus on the guideline for the treatment of non-tubal ectopic pregnancy, this book intends to fill this gap in the literature, compiling the best evidences in the medical literature guiding the reader on choosing the adequate treatment. An overview of each non-tubal ectopic pregnancy site will be presented in this introductory chapter. 


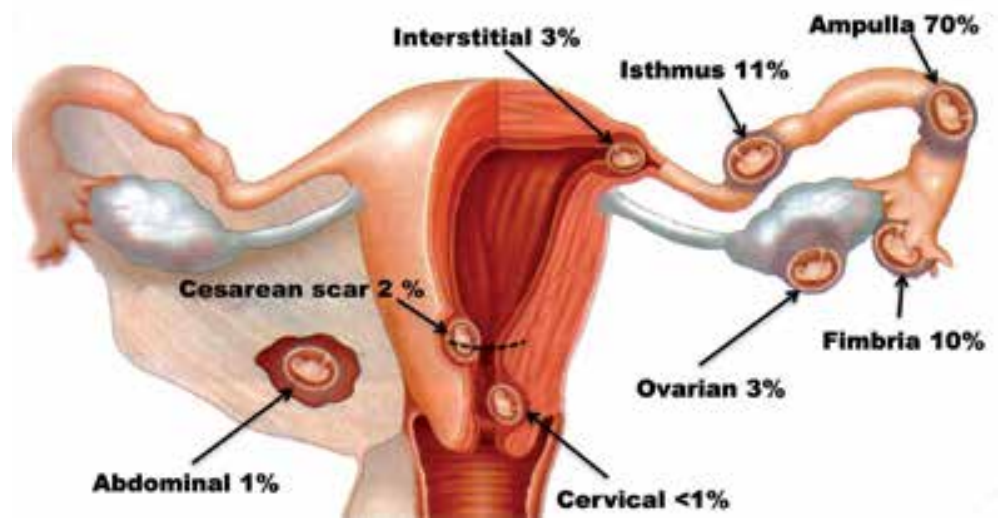

Figure 1.

Principal sites of ectopic pregnancy.

\section{Ovarian pregnancy}

It represents 3\% of ectopic pregnancies [3]. The sonographic aspect of ovarian pregnancy may range from gestational sac containing embryonic structures to solid and complex masses similar to those of tubal pregnancy. The major diagnosis difficulty is due to the fact that the pregnancy develops in the intimacy of the ovarian parenchyma, losing the reference used in tubal pregnancy, which is to identify the ovaries and then look for the pelvic mass. In the case of tubal pregnancy, it is imperative to demonstrate the ipsilateral ovary, as well as the adnexal mass, in order to decrease the possibility of false-positive diagnosis with ovarian masses. Therefore, ultrasound in this eventuality is not specific, once it may confuse ovarian pregnancy with other ovarian tumors. If gestational sac characteristics with embryonic structures are observed in the ovary, the diagnosis can be made with great precision [4].

Due to the difficulty of confirming the diagnosis noninvasively, most of the time, it is made during the intraoperative period. Thus, MTX treatment is used sporadically.

\section{Intraligamentary abdominal pregnancy}

Intraligamentary abdominal pregnancy is a rare form of ectopic pregnancy with a reported incidence of less than one in 250 ectopic pregnancies [5]. It usually results from the trophoblastic penetration of a tubal pregnancy through the serosa into the mesosalpinx, with secondary implantation between the leaves of the broad ligament. The placenta usually invades the intraligament space, ovary, uterus, omentum, pelvic peritoneum, and adjacent viscera [6].

Signs that may suggest intraligamentary abdominal pregnancy are abnormal vaginal bleeding, abdominal pain, painful fetal movements, easy palpation of the fetal parts, excessive nausea and vomiting, evidence of intrauterine growth restriction, and oligoamnios. The main antenatal complications include abdominal pain, gestational sac rupture with hemorrhage to the peritoneal cavity, vaginal bleeding, anomalous presentation, placental insufficiency, and fetal death.

During surgery, the placenta should preferably be removed to reduce the risk of peritonitis, abscess, disseminated intravascular coagulation, and persistent trophoblastic disease. 
Introductory Chapter: An Overview of the Diagnosis and Treatment of Non-tubal Ectopic... DOI: http://dx.doi.org/10.5772/intechopen.90905

Intraligamentary pregnancy is a condition of high maternal morbidity and mortality, and a judicious preoperative evaluation and surgical technique are imperative for a favorable outcome.

\section{Abdominal pregnancy}

It constitutes of about $1.5 \%$ of ectopic pregnancies [7]. It represents a risk of maternal death 7.7 times higher than that of tubal pregnancy and 90 times higher than that of intrauterine pregnancy [8]. The blastocyst can be implanted anywhere in the abdomen and in the different organs covered by the visceral peritoneum. Thus, in abdominal pregnancy, there is the development of the pregnancy in the peritoneal cavity. It can be classified into primary or secondary. Primary abdominal pregnancy is rare; most are secondary due to tubal rupture or tubal abortion. Few fetuses survive in the abdominal cavity and advance beyond the second trimester of pregnancy. The diagnostic and therapeutic difficulties are remarkable regardless of the location of advanced abdominal pregnancy.

The most frequent ultrasound findings in abdominal pregnancy are as follows [9]:

- Uterus separated from fetus (90\%)

- Extrauterine placenta (75\%)

- Oligoamnium (45\%)

- Fetal parts near the abdominal wall (25\%)

- Absence of myometrium between fetus/placenta and bladder (15\%)

- Anomalous fetal presentation (25-70\%)

- Difficulty viewing the placenta (25\%)

- Maternal intestinal loops obscuring fetal visualization (25\%)

- Fetal anomalies (20-40\%)

- Restricted intrauterine growth

- Lack of communication between endocervical canal and gestational sac

The most important signs are overlooked by the examiner because some unusual findings do not draw the examiner's attention to the diagnosis of abdominal pregnancy. For example, uterine walls should be visualized even if attention is focused entirely on fetal evaluation, as myometrial tissue may not be identified. Given the clinical suspicion of abdominal pregnancy (the mother reports feeling the child superficially in the abdomen), ultrasound becomes mandatory, but will not always be able to confirm the diagnosis accurately. Magnetic resonance imaging can confirm the diagnosis.

Fetal survival in abdominal pregnancy is the exception rather than the rule, and the live-born fetus is often malformed. As conditions for the concept are precarious, they succumb most of the time. In abdominal pregnancy, perinatal mortality ranges 
from 85 to $95 \%$ and maternal mortality is around 3\% [10]. Anomalies occur in about $1 / 3$ to $1 / 4$ of the fetuses whose viability is possible.

As pregnancy progresses, the placenta develops in any portion or organ of the abdominal cavity. We often observe digestive symptoms of subocclusion and excessive abdominal pain to fetal movements. The superficiality of the fetus is clear on palpation, as well as the auscultation of the fetal heartbeat. Ultrasound may demonstrate that the uterus is empty and compressed by the fetus and placenta.

As fetal survival is the exception, many advise against hospitalization to await the viability of the fetus. In addition, the risk of life-threatening bleeding is high.

In cases of late diagnosis and with the fetus alive, the follow-up may be to wait until the 36th week. In the presence of a dead fetus or living fetus after the 36th week, laparotomy is required. Antenatal diagnosis of abdominal pregnancy is essential for proper planning of the procedure. Precise placement of the placenta can be done more accurately by magnetic resonance imaging (MRI). Preoperative care should be individualized for each case according to its severity. Interventional radiology can be used with the placement of balloon catheters to prevent significant bleeding during surgery. In cases of dead fetus, selective embolization of the placental vessels may be performed. In addition, a multiprofessional staff may be required to perform insertion of ureteral catheters (double J), bowel preparation or transfusion blood reserve. An appreciable volume of blood and catheterized veins should be available to allow large volume to be infused rapidly, as well as a central venous pressure control and diuresis. In surgery, once the fetus is removed, the placenta is observed and, in particular, the site of its implantation. In cases where the placenta is attached to large vessels and the diagnosis is only made during surgery, the placenta could be left to prevent massive bleeding and the cord removed very close to its implantation site. This attempt should be considered to transfer the patient to a tertiary hospital. Evidently, there is a possibility of complications, infection, abscess formation, bruising, and intestinal obstruction.

\section{Interstitial pregnancy}

Interstitial pregnancy is defined as an ectopic pregnancy that is implanted in the interstitial portion of the fallopian tube. The interstitial portion is thick, $0.1-0.7 \mathrm{~mm}$ in diameter and $1-2 \mathrm{~cm}$ in length, and this part of the tube has a greater capacity to expand before rupture than the other segments of the tube [11]. Therefore, some cases are asymptomatic until the end of the first trimester of gestation and rupture may occur resulting in severe hemorrhage [12]. Because of the rich vascular anastomosis of the uterine and the ovarian arteries in this region, there may be accentuated hemorrhage. For this reason, early diagnosis is essential to reduce morbidity and mortality.

Interstitial pregnancies account for only $2-4 \%$ of ectopic pregnancies. However, the mortality rate is $2.5 \%$ [13]. This is because of the difficulty to confirm the diagnosis. Therefore, the late presentation could result in rupture and hemorrhage.

Interstitial pregnancy sometimes is incorrectly confused with cornual. Cornual pregnancy refers to a pregnancy in a horn of a bicornuate uterus or a rudimentary noncommunicating cavity horn or other Mullerian anomalies. The clinical outcome of cornual pregnancy varies greatly, depending on the size and expansile nature of the affected horn.

Risk factors for interstitial pregnancy are previous ectopic pregnancy, previous ipsilateral or bilateral salpingectomy, conception after in vitro fertilization, and history of sexually transmitted disease [14]. The symptoms of interstitial pregnancy 
are pelvic pain and vaginal bleeding in the first trimester of pregnancy. On physical examination, an asymmetric uterine enlargement may be palpable. Signs of acute abdomen may occur in interstitial pregnancy rupture with hemoperitoneum; in some cases, tachycardia and hypotension may be present.

The following are the ultrasound features for the diagnosis of interstitial pregnancy are (Figure 2):

- Interstitial line (echogenic line between the gestational sac in the interstitial region and the endometrial cavity)

- Eccentrically located gestational sac and myometrial thickness less than $5 \mathrm{~mm}$

It is important to point out that some viable intrauterine pregnancies are visualized by ultrasound in its early stages, in one of the lateral angles of the uterine cavity, medial to the uterotubal junction. This situation usually evolves to a viable intrauterine pregnancy and in some cases the incorrect diagnosis could lead to an unnecessary pregnancy interruption.

Classical treatments for interstitial pregnancy are resection of the interstitial portion of the tube or hysterectomy. If the diagnosis is made before rupture, minimally invasive surgery and nonsurgical treatment options can be used. Conservative options include methotrexate administration (local and systemic), expectant management, and minimally invasive surgical techniques that include resection of the involved interstitial portion of the tube and preserving the uterine architecture. The potential advantage of clinical treatment is to avoid a surgical scar on the uterus and the risks associated to surgery.

Expectant management that has been practiced based on the natural course of many early EPs is a self-limiting process [16]. The patients selection for this management are hemodynamically stable patients, beta-hCG $\leq 2000 \mathrm{mUI} / \mathrm{ml}$, decline

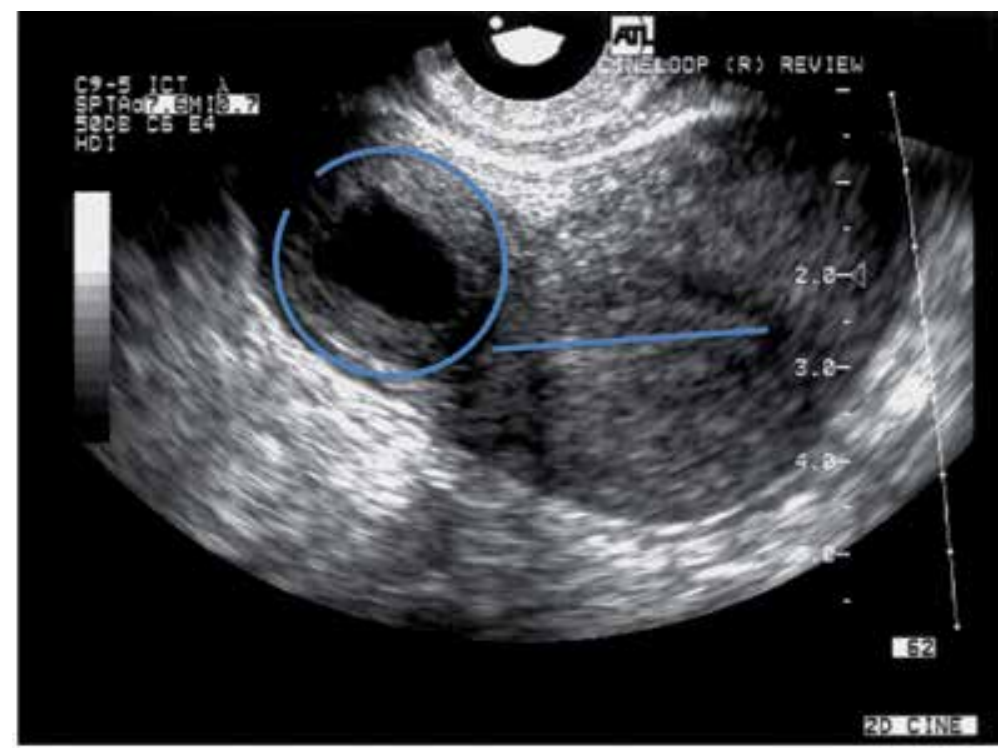

Figure 2.

Interstitial ectopic pregnancy: Presence of an eccentric gestational sac with myometrial tissue that surrounds the gestational sac with a thickness of $2 \mathrm{~mm}$ and the interstitial line [15]. 
of the titers of beta-hCG in an interval of $24 / 48 \mathrm{~h}$, and interstitial pregnancy mass $\leq 3.5 \mathrm{~cm}$ and without fetal cardiac activity.

Systemic methotrexate treatment was used for the first time in an interstitial pregnancy. In 1982, Tanaka et al. [17] successfully treated an interstitial pregnancy using methotrexate. Ectopic interstitial pregnancies without embryonic cardiac activity and beta-hCG $\leq 5000 \mathrm{mUI} / \mathrm{ml}$ should be treated using a single dose of MTX $50 \mathrm{mg} / \mathrm{m}^{2} \mathrm{IM}$. Cases with beta-hCG > $5000 \mathrm{mUI} / \mathrm{ml}$ should be treated with a protocol of multiple doses of MTX. On the other hand, cases with embryonic heart activity should be treated with transvaginal administration of MTX under sonographic guidance.

\section{Cervical pregnancy}

Cervical ectopic pregnancy is defined by the implantation and development of the conceptus in the cervical canal. Among all ectopic pregnancies, the cervical pregnancy is the rarest, representing about $0.4 \%$ of cases [1]. It is accompanied by high morbidity and mortality, which can lead to severe hemorrhage, due to the rich vascularization of the cervix and a small amount of muscle fibers. The etiology is not well established; however, some predisposing factors were related, such as previous uterine curettages and cesarean section, Asherman syndrome, history of uterine and cervical surgeries, and in vitro fertilization. The diagnosis is made through history and physical examination and confirmed by ultrasound. Patients with menstrual delay and positive pregnancy tests may be asymptomatic, complain of vaginal bleeding, or even have severe vaginal bleeding. On vaginal examination, the cervix will be enlarged and congested, with painful tumoration. In addition, however, complaints and physical examination are often nonspecific, making clinical diagnosis difficult.

Early diagnosis made by ultrasound contributes to the success of conservative therapies. Ultrasound findings include empty uterine cavity; endometrial deciduous reaction; hourglass-shaped uterus; enlarged cervical canal; gestational sac within the canal, with or without heartbeat; placental tissue surrounding the gestational sac; and closed internal os of the cervix. These findings can also be visualized in MRI with more accuracy (Figure 3).

If the clinical condition is unstable due to the hemorrhagic situation, it is valid to use the Foley catheter into the cervix insufflating from 30 to $100 \mathrm{ml}$ while the patient is stabilized and then to evaluate the need for a hysterectomy.

With the development of conservative treatment protocols, the need for hysterectomies has decreased from $89.5 \%$ before 1987 to $21 \%$ in 1994 . Conservative treatment options can be categorized as tamponade, which is performed with intracervical balloon after curettage; cerclage to reduce cervical artery blood flow associated with manual intrauterine cervical aspiration; reduced blood supply through embolization or uterine artery ligation; excision of trophoblastic tissue by hysteroscopic resection; intra-amniotic feticide, by local injection of methotrexate; and systemic chemotherapy with intramuscular methotrexate.

In cases of embryo without heartbeat with high beta-hCG titers, multiple dose of systemic MTX treatment is the preferred therapeutic option. When the embryo presents heartbeat, local treatment with MTX is indicated. Elito et al. [18] published a series of eight cases of cervical pregnancy with live embryo treated with transvaginal US-guided gestational sac puncture and MTX injection ( $1 \mathrm{mg} / \mathrm{kg})$. All patients were successfully treated. The initial beta-hCG ranged from 3013 to 71,199 $\mathrm{mIU} / \mathrm{mL}$. Only one case evolved to infection. There was no need for further interventions in this case series. The time interval for beta-hCG titers to be negative was 


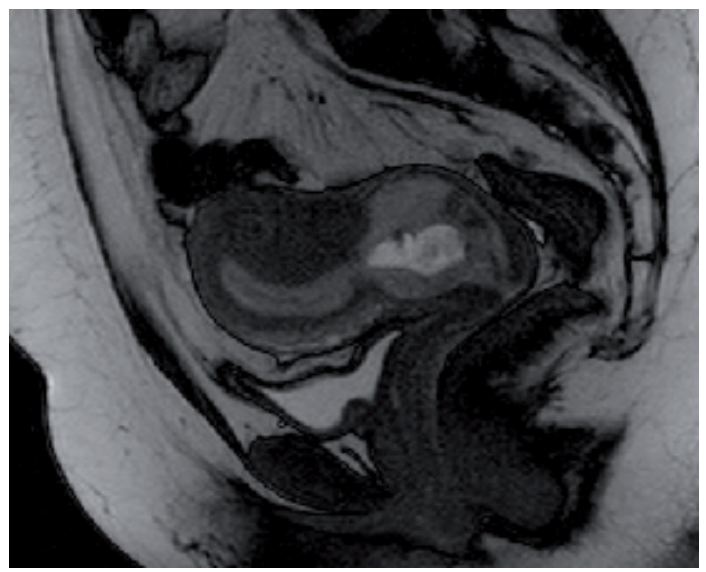

Figure 3.

Magnetic resonance image showing cervical pregnancy, hourglass-shaped uterus, empty uterine cavity, and cervix with heterogeneous mass inside. (author image file).

2-12 weeks. The period for the disappearance of the image of the ectopic pregnancy assessed by ultrasound was 3-14 weeks. Two patients had subsequent intrauterine pregnancies. The authors concluded that conservative treatment of cervical ectopic pregnancy with live embryos treated with transvaginal US-guided puncture and MTX injection is effective and avoids other mutilating interventions.

\section{Cesarean scar pregnancy}

Pregnancy in cesarean section scar is the rarest form of ectopic pregnancy. From the first case described in 1978 [19] to 2001, there were only 19 reported cases [20]. In 2006, there were 155 cases and in 2011 the number of cases described in the literature was 751, which shows a rapid increase in the incidence of this type of pregnancy. The estimated incidence is from 1 in 1800 to 1 in 2216 pregnancies, with a rate of $6.1 \%$ of all ectopic pregnancies in women with a history of previous cesarean section [1].

The basis of the pathophysiology is the invasion of the blastocyst in the myometrium through minimal communication between the anterior cesarean section scar and the endometrial cavity.

Risk factors are previous cesarean sections, short time between cesarean section and current pregnancy, IVF treatment, and retroverted uterus, which may lead to greater cesarean section scar dehiscence increasing the chance of implantation of the gestational sac in this region.

The cesarean scar ectopic pregnancy tends to behave more aggressively because of the risk of uterine rupture and bleeding in the first and second trimesters of pregnancy.

Transvaginal ultrasound allows early diagnosis of the disease before these tragic outcomes and allows conservative treatment rather than mutilating surgeries such as hysterectomy and then preserving fertility. It also allows differential diagnosis with ongoing abortion, molar pregnancy, and ectopic cervical pregnancy.

The diagnosis can be made by ultrasonography (Figure 4) and magnetic resonance imaging (Figure 5).

Transvaginal ultrasound findings for cesarean scar pregnancy include empty uterine cavity, decidual reaction, trophoblast located at the site of previous cesarean scar, and absence of healthy myometrium between the bladder and gestational sac. 


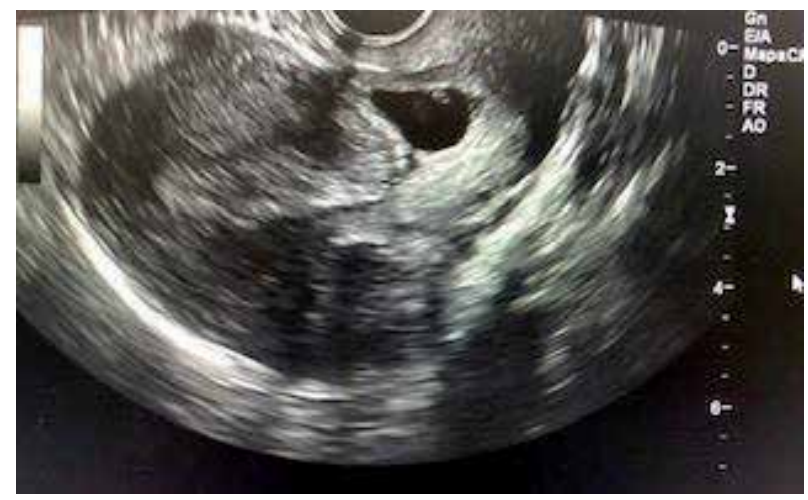

Figure 4.

Cesarean scar pregnancy in the isthmic region with the presence of gestational sac with embryo. (author image file).

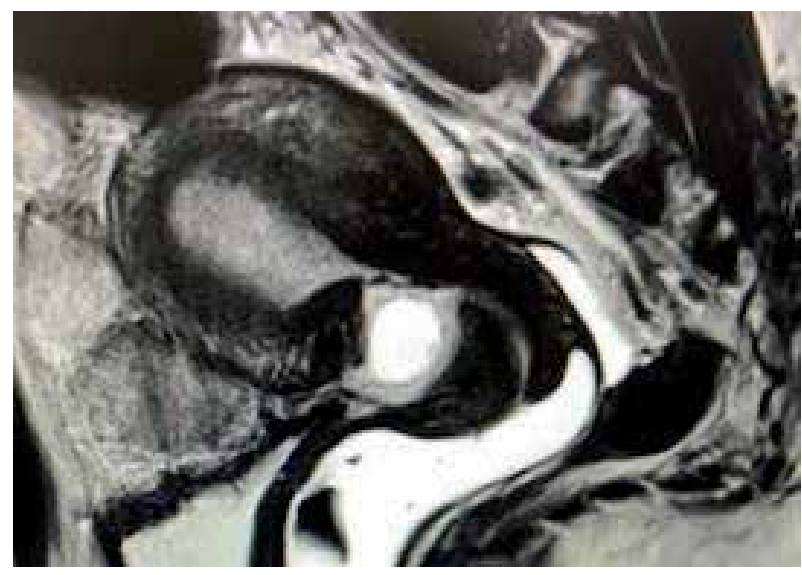

Figure 5.

MRI of the uterus demonstrating an empty uterus, an empty cervical canal, the gestational sac being located in the anterior part of the isthmic portion of the uterus with a diminished myometrial layer, and a discontinuity in the anterior wall of the uterus.

There are two types of ectopic pregnancy in cesarean scar section: endogenous and exogenous. In the endogenous type, gestational sac implantation occurs in the cesarean section scar, with the development of pregnancy toward the uterine cavity. The exogenous type occurs with deeper implantation of the gestational sac in the cesarean scar, and as the pregnancy develops in the direction of the bladder, it increases the risk of rupture and hemorrhage in the first trimester of pregnancy.

The relationship between the gestational sac of a cesarean scar pregnancy and the endometrial line is defined as crossover sign (COS). In this sonographic sign, a straight line was drawn connecting the internal cervical os and the uterine fundus through the endometrium (endometrial line). The gestational sac was identified and its diameter, perpendicular to the endometrial line, was traced. Patients were categorized according to the relationship between the endometrial line and the diameter of the gestational sac into two groups: (1) COS-1, in which the gestational sac was implanted within the cesarean scar, and at least two-thirds of the diameter of the gestational sac was above the endometrial line, toward the anterior uterine wall; and (2) COS-2, in which the gestational sac was implanted within the cesarean scar, and less than two-thirds of the gestational sac was above the endometrial line [21] (Figure 6). 

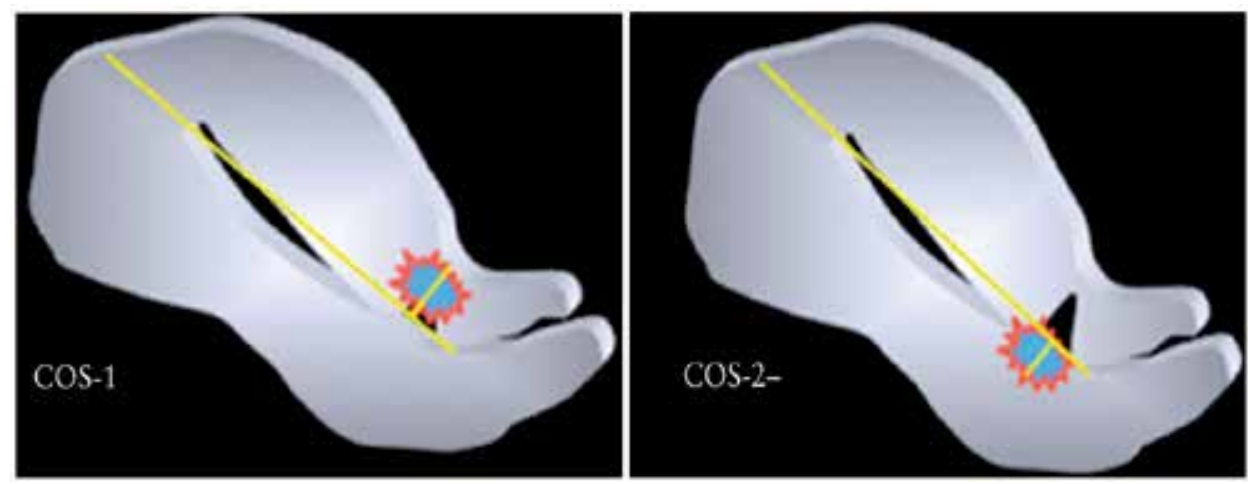

Figure 6.

Crossover sign representing COS 1 , in which the gestational sac was implanted within the Cesarean scar, and at least two-thirds of the diameter of the gestational sac was above the endometrial line, towards the anterior uterine wall; and COS-2, in which the gestational sac was implanted within the Cesarean scar, and less than two-thirds of the gestational sac was above the endometrial line [21].

This crossover sign may help to determine whether a cesarean scar pregnancy will progress to rupture in the first trimester (COS-1), or in a COS-2 case, the pregnancy could continue running the risk of placenta accreta and cesarean hysterectomy [21].

Approaches are divided into surgical and nonsurgical treatment. Surgical treatment may include uterine curettage or hysterectomy and uterine artery embolization. Nonsurgical treatment may be expectant or medical treatment with systemic or local methotrexate.

There is still no consensus on the best mode of treatment in case of ectopic pregnancy in cesarean scar section.

Surgical intervention is performed in the presence of important bleeding and, under these circumstances, if possible, a uterine artery embolization attempted before curettage or hysterectomy.

Dilatation and curettage with subsequent intrauterine Foley catheter insertion may be an option due to the simplicity. However, the risk of this treatment is the hemorrhage and the necessity of secondary hysterectomy. This treatment should only be used in selected cases.

Excision by hysteroscopy is another option. During the procedure, the pelvic ultrasound could help to avoid complications.

Excision by laparotomy or laparoscopy could be attempted. These procedures usually are performed if local MTX treatment failed or after uterine rupture.

Hysterectomy is usually performed when other treatments failed and to control the bleeding (Figure 7). This procedure is not the first line for the majority of gynecologists.

When the patient presents a cesarean scar pregnancy COS-2 or endogenous type and it is a viable pregnancy, the patient should be advised about the risks of continuing with the pregnancy. The late diagnosis of cesarean scar pregnancy evolves to a placenta accreta. The risks of continuing the pregnancy are hemorrhage (13\% in first and second trimesters, and 39\% in third trimester), uterine rupture (9.9\% in first and second trimesters, and $10.2 \%$ in third trimester), placenta accreta $75 \%$, and hysterectomy $(15.2 \%$ in first and second trimesters, and $60.6 \%$ in the third trimester) [22]. The physician should discuss these risks and advise the patient. A deep reflection of the situation is required before taking the decision to keep or not the pregnancy, once the couple usually already has one child. When the decision is to continue with the pregnancy, some criteria 


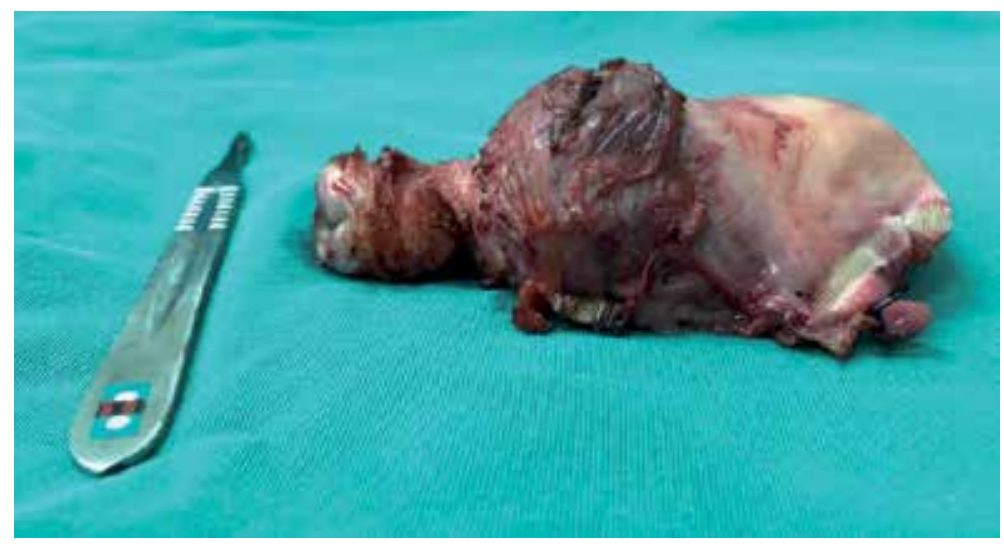

Figure 7.

Case of cesarean scar pregnancy evolved to hemorrhage and the patient was submitted to hysterectomy.

should be evaluated. In cases that have crossover sign (COS-1) or exogenous type and the magnetic resonance shows myometrium less than $5 \mathrm{~mm}$, the pregnancy should be interrupted. In the other situation COS-2 or endogenous type and myometrium thickness $>5 \mathrm{~mm}$, if the patient opts to continue with the pregnancy, they should be advised about the risk of placenta accreta and cesarean hysterectomy and should be assisted in a hospital with interventionist radiology and neonatal intensive care.

Systemic methotrexate treatment is not effective in cases where the embryo has heartbeat. This treatment should be avoided in this condition because it delays a more effective treatment.

In cesarean scar pregnancy when the embryo/fetus has heartbeat, local methotrexate $(1 \mathrm{mg} / \mathrm{kg})$ treatment guided by transvaginal ultrasound should be the first option [23] (Figures 8 and 9). The current scientific evidences demonstrate that local treatment with MTX brings best results. This type of treatment is broadly used because it is not only effective but also simple and low cost.

In cases where the embryo/fetus has no heartbeat, it is important to check the titers of beta-hCG in an interval of 24/48 hours. If the levels were low and declining, the best approach is the expectant management. However, if the titers were high and increasing, the patient should be submitted to the systemic treatment with MTX. If the levels of beta-hCG are low (less than $5.000 \mathrm{mUI} / \mathrm{ml}$ ), a single dose of MTX $50 \mathrm{mg} / \mathrm{m}^{2}$ (same treatment of tubal pregnancy) is recommended. On the other hand, if the levels are higher $(>5.000 \mathrm{mUI} / \mathrm{ml})$, a protocol of multiple doses of MTX (1 mg/kg) on days 1, 3, 5, and 7 and folinic acid $(0.1 \mathrm{mg} / \mathrm{kg})$ on days 2, 4, 6, and 8 is recommended.

The MTX treatment is an outpatient follow-up with weekly examinations of $\beta$-hCG until resolution. During outpatient follow-up, ultrasound is not routinely performed and should only be repeated when $\beta$-hCG reaches a negative value 3 months in a row. Other than that the ultrasound should be repeated during the treatment in any case of complication suspected. In general, after 3-6 months, the image of the ectopic pregnancy disappears, and at this point, it is recommended to perform hysterosalpingography and hysteroscopy for a better evaluation of the uterine cavity, as well as appropriate reproductive planning for these patients [1].

In cases where the resolution takes a long period, other treatments could be offered, such as gestational sac aspiration under ultrasound or hysteroscopy to remove the gestational tissue with vascular coagulation of the implantation site. 
Introductory Chapter: An Overview of the Diagnosis and Treatment of Non-tubal Ectopic... DOI: $h$ ttp://dx.doi.org/10.5772/intechopen.90905

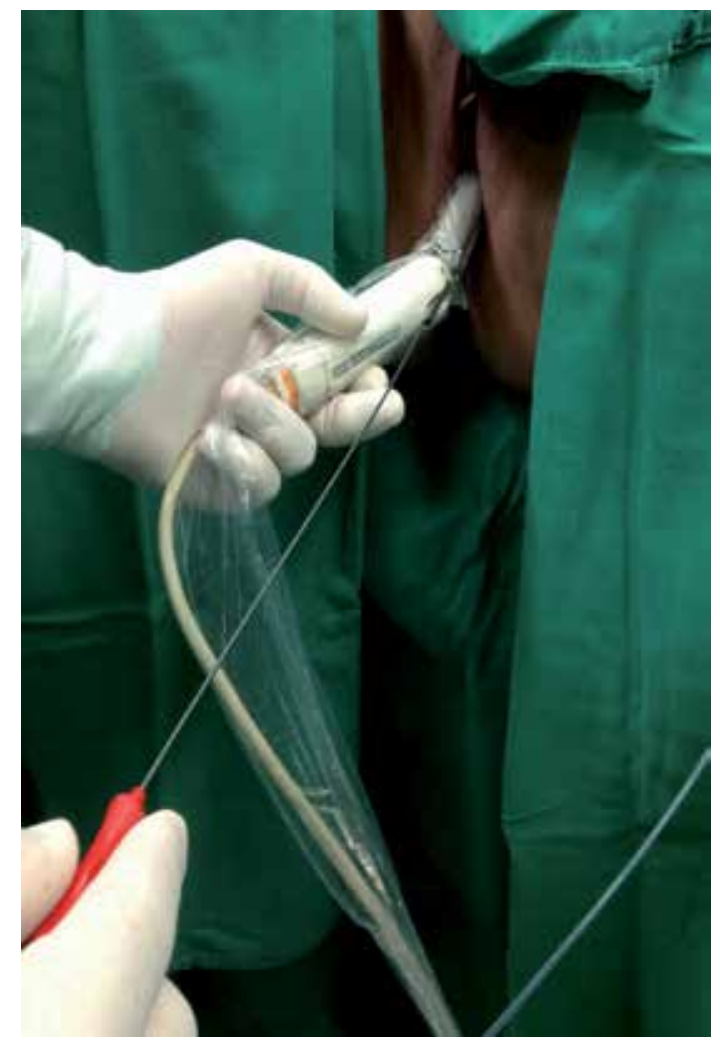

Figure 8.

The patient in the lithotomy position. A 22-gauge, $15 \mathrm{~cm}$ Wallace needle was inserted via vaginal route under transvaginal ultrasound guidance.

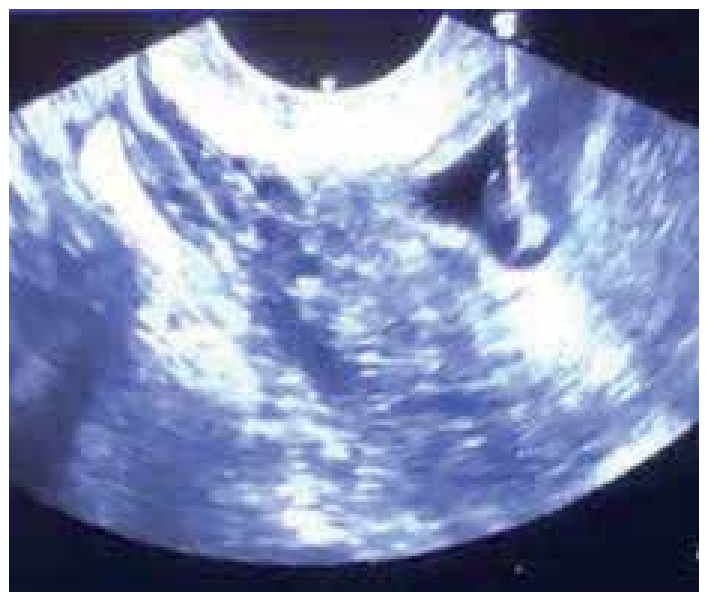

Figure 9.

Wallace needle was inserted via vaginal route into the gestational sac. MTX (1 mg/ $\mathrm{kg})$ was injected into the gestational sac.

In cases where local methotrexate treatment fails, uterine artery embolization should be considered [24], or laparoscopic or laparotomic cesarean scar pregnancy wedge resection with subsequent surgical correction of the cesarean section scar.

Birch et al. [25] in a systematic review about treatments for cesarean scar pregnancy showed that uterine artery embolization (UAE) combined with D\&C had good results, because it presented a low risk of heavy bleeding and hysterectomy. 

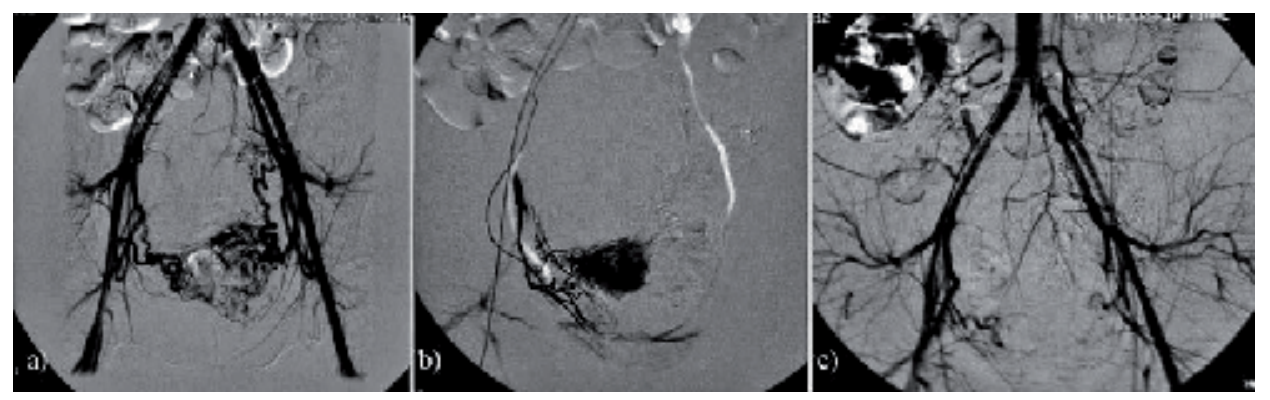

Figure 10.

Bilateral uterine angiography before and after uterine artery embolization (a) uterine arteries were enlarged by means of hypervascular infusion of methotrexate before uterine artery embolization, (b) unilateral occlusion was successfully performed, (c) bilateral arterial occlusion was confirmed after the uterine artery embolization and no extravasation was observed [26].

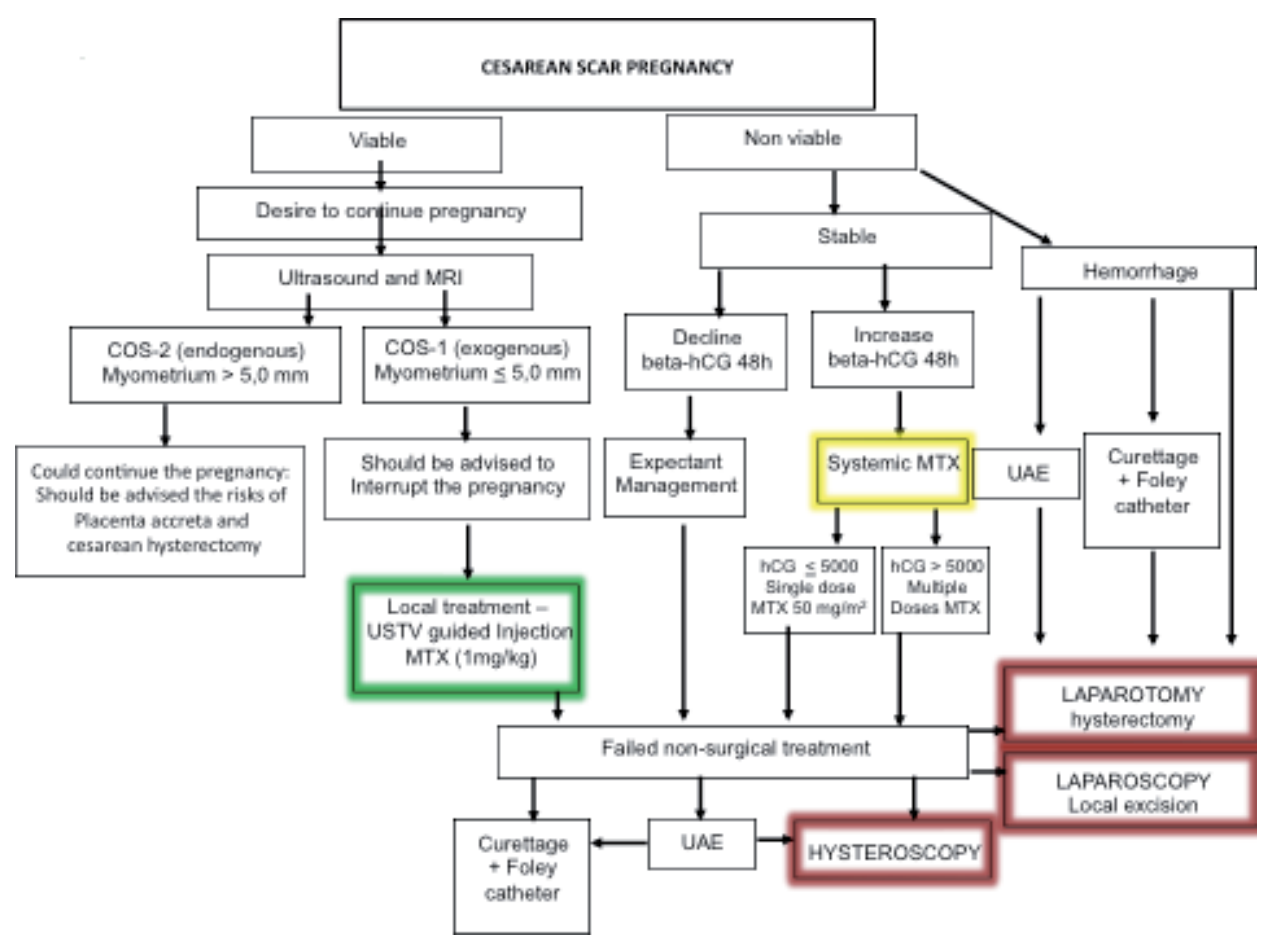

Figure 11.

The management protocol of cesarean scar pregnancy.

The UAE procedure can be performed under spinal anesthesia and conscious sedation with standard antibiotic prophylaxis. After asepsis of groin area, the right or left common femoral artery is cannulated via the Seldinger technique, introducer $5 \mathrm{~F}$, and the procedure continues such as in the uterine fibroid embolization, with catheterization of the left uterine artery and subsequently the right uterine artery. Methotrexate is injected transarterially prior to the embolization with occluding microparticles, always larger than $500 \mu$ to avoid paradoxical embolization to the ovary [26]

(Figure 10). Despite arterial occlusion, the risk of severe bleeding is non-negligible because of gradual restoration of the normal circulation within approximately 3 weeks. The dilation and curettage with suction or vacuum aspiration 6-8 hours after intra-arterial chemoembolization reduce the incidence of bleeding [25]. 
There is a lack of consensus on the guideline for the treatment of cesarean scar pregnancy and several options of treatment are proposed in the literature (isolated or combined) [27]. Therefore, the author prepared a protocol to manage cesarean scar pregnancy based on his experience and the scientific evidences collected until now (Figure 11).

\section{Nonsurgical treatment in non-tubal pregnancies}

Management in cases of interstitial, cervical, and cesarean scar pregnancy should always be individualized. Nonsurgical treatment has emerged as an important alternative for these situations, avoiding surgeries that compromise the reproductive future. When the embryo has heartbeat, USTV-guided local treatment with MTX injection into the gestational sac at a dose of $1 \mathrm{mg} / \mathrm{kg}$ is the first-line treatment. When beta-hCG titers are greater than $5000 \mathrm{mIU} / \mathrm{ml}$, the local treatment is complemented with the systemic multiple-dose protocol starting the day after puncture. Systemic treatment with MTX is performed in cases where the embryo has no heartbeat. The medical treatment will depend on the initial beta-hCG titer. For titers below $5000 \mathrm{mIU} / \mathrm{ml}$, a single dose of MTX $50 \mathrm{mg} / \mathrm{m}^{2} \mathrm{IM}$ is recommended. On the other hand, if beta-hCG titers are greater than $5000 \mathrm{mIU} / \mathrm{ml}$, a multiple dose MTX protocol is recommended. The MTX dose is $1 \mathrm{mg} / \mathrm{kg}$ IM on days $1,3,5$, and 7, alternating with folinic acid at the dose of $0.1 \mathrm{mg} / \mathrm{kg}$ IM on days $2,4,6$, and 8 (Figure 12).

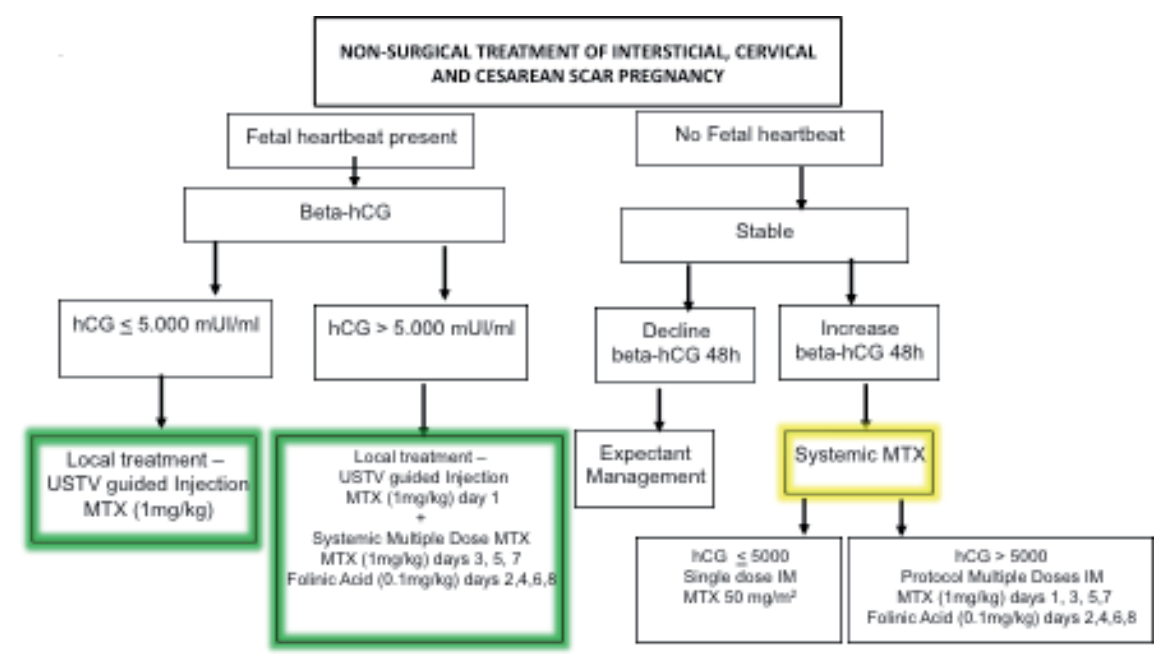

Figure 12.

Nonsurgical protocol of non-tubal pregnancy.

\section{Final considerations}

Non-tubal pregnancy is a challenge for obstetricians due to the maternal morbidity and mortality. The incidence is increasing and the physicians should be aware of this situation. The early diagnosis by ultrasound is essential for a minimal invasive treatment avoiding fertility loss. There are several options for the treatment and no consensus in medical literature. In this introductory chapter, surgical and nonsurgical approaches as well as guidelines have been presented for the treatment of non-tubal pregnancy based on current scientific evidence. 


\section{Author details}

Julio Elito Jr

Federal University of Sao Paulo, Brazil

*Address all correspondence to: elitojjr@hotmail.com

\section{IntechOpen}

(C) 2020 The Author(s). Licensee IntechOpen. This chapter is distributed under the terms of the Creative Commons Attribution License (http://creativecommons.org/licenses/ by/3.0), which permits unrestricted use, distribution, and reproduction in any medium, provided the original work is properly cited. (cc) BY 


\section{References}

[1] Cecchino GN, Araujo Júnior E, Elito Júnior J. Methotrexate for ectopic pregnancy: When and how. Archives of Gynecology and Obstetrics.

2014;290(3):417-423

[2] Shen L, Fu J, Huang W, Zhu H, Wang Q, Yang S, et al. Interventions for non-tubal ectopic pregnancy. Cochrane Database of Systematic Reviews. 2014;7:CD011174

[3] Chetty M, Elson J. Treating nontubal ectopic pregnancy. Best Practice and Research. Clinical Obstetrics and Gynaecology. 2009;23:529-538

[4] Comstock C, Huston K, Lee W. The ultrasonographic appearance of ovarian ectopic pregnancies. Obstetrics and Gynecology. 2005;105(1):42-46

[5] Phupong V, Terasakul P, Kankaew K. Broad ligament twin pregnancy. A case report. The Journal of Reproductive Medicine. 2001;46(2):144-146

[6] Holzhacker S, Elito J Jr, Santana RM, Hisaba W. Advanced intraligamentary abdominal pregnancy-Case Report. Revista da Associacao Medica Brasileira (1992). 2008;54(5):387-389

[7] Bouyer J, Coste J, Ferrnandez H, et al. Sites of ectopic pregnancy: A 10 year population-based study of 1800 cases. Human Reproduction. 2002;17:3224-3230

[8] Centre for Maternal and Child Enquiries (CMACE). 2006-2008 saving mothers' lives. Reviewing maternal deaths to make motherhood safer. British Journal of Obstetrics and Gynaecology. 2011;118(Suppl 1):1-203

[9] Martin JJ, Sessums JK, Martin RW. Abdominal pregnancy: Current concepts of management. Obstetrics and Gynecology. 1988;71:549
[10] Stevens CA. Malformations and deformations in abdominal pregnancy. The American Journal of Medicine. 1993;47:1189

[11] Eddy CA, Pauerstein CJ. Anatomy and physiology of the fallopian tube. Clinical Obstetrics and Gynecology. 1980;23:1177

[12] Lau S, Tulandi T. Conservative medical and surgical management of interstitial ectopic pregnancy. Fertility and Sterility. 1999;72:207-215

[13] Rock JA, Damario MA. Ectopic pregnancy. In: Rock JA, Thompson JD, editors. TeLinde's Operative Gynecology. 8th ed. Philadelphia, PA, USA: Lippincott-Raven; 1997. pp. 505-520

[14] Tulandi T, Al-Jaroudi D. Interstitial pregnancy: Results generated from the Society of Reproductive Surgeons Registry. Obstetrics and Gynecology. 2004;103(1):47-50

[15] Elito J Jr. Clinical treatment of unruptured ectopic pregnancy. Chapter 11. In: Kamrava M, editor. Ectopic Pregnancy: Modern Diagnosis and Management. 1st ed. Croatia: IntechOpen; 2011. pp. 177-208

[16] Zalel Y, Caspi B, Insler V. Expectant management of interstitial pregnancy. Ultrasound in Obstetrics and Gynecology. 1994;4(3):238-240. PubMed PMID: 12797190

[17] Tanaka T, Hayashi H, Kutsuzawa T, Fujimoto S, Ichinoe K. Treatment of intersticial ectopic pregnancy with methotrexate: Report of a successful case. Fertility and Sterility. 1982;37:851-855

[18] Júnior JE, Musiello RB, Araujo Júnior E, Souza E, Fava JL, Guerzet EA, et al. Conservative management of cervical pregnancy with embryonic 
heart activity by ultrasound-guided local injection: An eight case series. The Journal of Maternal-Fetal and Neonatal Medicine. 2014;27(13):1378-1381

[19] Larsen JV, Solomon MH. Pregnancy in a uterine scar sacculus-An unusual cause of postabortal haemorrhage. A case report. The South African Medical Journal. 1978;53(4):142-143

[20] Ash A, Smith A, Maxwell D. Caesarean scar pregnancy. BJOG: An International Journal of Obstetrics and Gynaecology. 2007;114(3):253-263

[21] Cali G, Forlani F, Timor-Tritsch IE, Palacios-Jaraquemada J, Minneci G, D'Antonio F. Natural history of cesarean scar pregnancy on prenatal ultrasound: The crossover sign. Ultrasound in Obstetrics and Gynecology. 2017;50(1):100-104

[22] Calì G, Timor-Tritsch IE, PalaciosJaraquemada J, Monteaugudo A, Buca D, Forlani F, et al. Outcome of cesarean scar pregnancy managed expectantly: Systematic review and meta-analysis. Ultrasound in Obstetrics and Gynecology. 2018;51(2):169-175

[23] Leite F, Fraietta R, Elito J Jr. Local management with methotrexate of cesarean scar ectopic pregnancy with live embryo guided by transvaginal ultrasound: A case report. Revista da Associacao Medica Brasileira (1992). 2016;62(2):184-185

[24] Fornazari VA, Szejnfeld D, Elito J Jr, Goldman SM. Interventional radiology and endovascular surgery in the treatment of ectopic pregnancies. Einstein (Sao Paulo). 2015;13(1):167-169

[25] Birch Petersen K, Hoffmann E, Rifbjerg Larsen C, Svarre Nielsen H. Cesarean scar pregnancy: A systematic review of treatment studies. Fertility and Sterility. 2016;105(4):958-967
[26] Elito J Jr, Araujo Júnior E, Martins Santana EF, Szejnfeld D, Helfer TM, Nardozza LM, et al. Uterine artery embolization with methotrexate infusion as treatment for cesarean scar pregnancy. Case report. Medical Ultrasonography. 2013;15(3):240-243

[27] Timor-Tritsch IE, Monteagudo A, Calì G, D’Antonio F, Agten AK.

Cesarean scar pregnancy: Patient counseling and management. Obstetrics and Gynecology Clinics of North America. 2019;46(4):813-828 
Section 2

Epidemiology 



\title{
Chapter 2
}

\section{Non-tubal Ectopic Pregnancy: Incidence and Diagnosis}

\author{
Aliye Nigar Serin and Özer Birge
}

\begin{abstract}
Ectopic pregnancies occur at 1-2\% of all pregnancies. The most common implantation site is the fallopian tube with 95 , and $5 \%$ are non-tubal located. The aim of this review is to determine the current state of data about the diagnosis and the treatment of non-tubal ectopic pregnancies. Literature is reviewed concerning cervical, interstitial, cornual, ovarian, Caesarean scar, and abdominal ectopic pregnancies from PubMed databases. Non-tubal ectopic pregnancies are often misdiagnosed and overlooked. Clinical symptoms and ultrasound must be combinated to diagnose. Management may involve medical treatment with methotrexate or surgery or a combination according to patient's clinical stability and the location of ectopic pregnancy. Non-tubal ectopic pregnancies are rare but can be a life-threatening condition due to late diagnosis. Early diagnosis and treatment of patients are associated with decreased morbidity and mortality in non-tubal pregnancy and, very importantly, preserve the uterus and subsequent fertility. İncreased experiences have led to choose the best way to manage non-tubal pregnancies and develop new techniques.
\end{abstract}

Keywords: non-tubal ectopic pregnancy, methotrexate, cervical, interstitial, cornual, ovarian, caesarean ectopic

\section{Introduction}

An ectopic pregnancy (EP) refers to the implantation of a pregnancy outside of the uterus cavity. The overall rate of EP is $1-2 \%$ in the general population and $2-5 \%$ among patients who have utilized assisted reproductive technology (ART) $[1,2]$. Up to $98 \%$ of ectopic pregnancies occur in the fallopian tubes. Non-tubal ectopic pregnancies are rare, accounting for $7-10 \%$ of all ectopic pregnancies and occurring outside the uterus and tubes $[3,4]$, yet are associated with higher morbidity due to their late presentation and diagnostic difficulties [5-8]. There are six main locations for non-tubal ectopic pregnancies that are cervical, interstitial, cornual, ovarian, Cesarean scar, and abdominal. The main risk factors for non-tubal pregnancy include previous ectopic, history of assisted reproduction, pelvic infections, smoking, and the use of the progesterone only pill or intrauterine device [9]. Early diagnosis and treatment of patients are associated with decreased morbidity and mortality in non-tubal pregnancy and, very importantly, preserve the uterus and subsequent fertility. 


\section{Cervical ectopic pregnancy}

Cervical pregnancies are rare accounting for less than $1 \%$ of all ectopic pregnancies, and the incidence is 1:2500-18,000 $[3,4,9]$. They result due to the risk of trophoblast penetration through the mucosa of cervical wall into the uterine vessels. Cervical ectopics may arise should the blastocyst pass through the uterine cavity and implant into the mucosa of the endocervical canal [10-13]. Almost 70\% of cases with subsequent cervical EP have a history of dilation and curettage (D\&C) in a previous pregnancy $[12,14]$. Also in vitro fertilization (IVF) seems as a risk factor but often jointly with D\&C and other possible risk factors; anatomic anomalies (myomas, synechiae), intrauterine device (IUD) use, and diethylstilbestrol exposure, although these are not strong associations, are difficult to isolate as an independent contributor to risk [15].

Vaginal bleeding without pain is the most common presenting symptom but in more advanced pregnancies may be coupled with abdominal pain and urinary problems. Examination findings include an enlarged, globular, or distended cervix, which is often associated with dilatation of the external os [12].

Before 1979, cervical pregnancy was almost always associated with hysterectomy because of out-of-control vaginal bleeding, and the primary diagnosis was made by histological analysis of the hysterectomized uterus [16]. Preoperative diagnosis was rarely possible. After the first ultrasound report of cervical pregnancy was published by Raskin in 1978, transvaginal ultrasonography has become the main diagnostic tool [17]. This put forward more conservative approaches that attempt to limit morbidity and preserve fertility. The majority of patients with a cervical pregnancy are women with low parity; thus, preservation of reproductive function is a priority [12].

Cervical pregnancy may appear as a hemorrhagic mass, gestational sac, or presence of a fetus (with or without cardiac activity) on TvUSG [10,12]. Defined sonographic criteria are shown in Table 1. A cervical EP is identified on ultrasound by a distended cervical canal containing a gestational sac (Figure 1), below a closed internal cervical os $[19,20]$, misdiagnosed as an intrauterine pregnancy with a low implantation site or a failed pregnancy imminent abortion. The "sliding sign" involves the sliding of the products of conception against the endocervical canal when gentle pressure by the sonographer during transvaginal ultrasound associated with spontaneous abortions in progress and should be absent in a cervical ectopic pregnancy [5, 6, 9].

The treatment for cervical ectopic pregnancy is unclear. If gestation is $<12$ weeks, with no fetal heart present and lower-serum hCG values, conservative management is most effective in women wishing to preserve fertility [21, 22].

Single- or multiple-dose systemic methotrexate (MTX) efficacy is $91 \%$, reported in a meta-analysis [21]. MTX is more successful in pregnancies $<9$ weeks and with beta hCG levels $<10,000 \mathrm{mIU} / \mathrm{mL}, \mathrm{CRL}<10 \mathrm{~mm}$, and absent fetal cardiac activity $[9,23]$. The folic acid antagonist methotrexate is the most widely used systemic chemotherapy.

\begin{tabular}{ll}
\hline Anatomical structure & Sonographic appearance \\
\hline Uterus & Empty \\
Cervix & Barrel shaped \\
Cestational sac & Below uterine arteries \\
Sliding sign & Absent \\
Doppler blood flow & Around sac \\
\hline
\end{tabular}

Table 1.

Sonagraphic criteria for cervical pregnancy. 


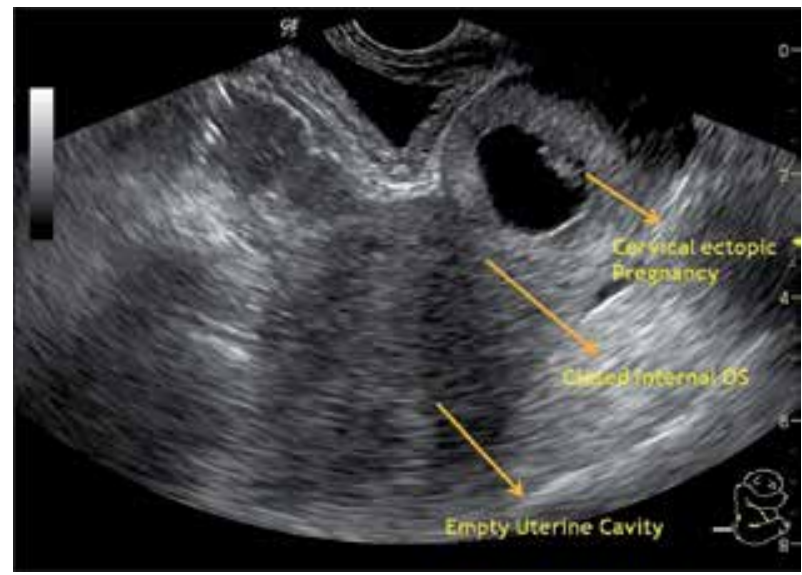

Figure 1.

Cervical ectopic pregnancy [18].

In cervical pregnancy with embryonic heart activity, the treatment of choice is local injection of methotrexate or potassium chloride by ultrasound-guided injection [24].

İn the treatment of cervical ectopic pregnancy, uterine artery ligation, uterine artery embolization, balloon tamponade, cervical curettage, cerclage, cervical stay sutures, and injection of prostaglandin F2a can be combined to control hemorrhage $[3,7,10,25]$.

Medical management must only be suggested if the patient is hemodynamically stable; otherwise, surgical treatment should be attempted as dilatation and evacuation, hysteroscopic resection, and hysterectomy.

\section{Interstitial ectopic pregnancy}

The incidence of interstitial pregnancy is $1-11 \%$ of all ectopic pregnancies. It has a high complication and maternal mortality rate, approximately $20 \%$ of all deaths caused by ectopic pregnancies [3-5]. The pregnancy implants at the junction of the interstitial part of the fallopian tube and the uterine myometrium. The main risk factor for interstitial implantation is prior ipsilateral salpingectomy; a residual "stump" of tube may form the focus of ectopic pregnancy development [26]. Symptoms are amenorrhea or spotting with or without abdominal pain.

Diagnostic criteria by ultrasound include:

1. Myometrial tissue $<5 \mathrm{~mm}$ thick surrounded by gestational sac [26] (Figure 2).

2. An echogenic line between the gestational sac in the cornua and endometrial cavity named "interstitial line" has a sensitivity of $80 \%$ and specificity of $98 \%$ [4-6, 28] (Figure 3).

3. Empty uterine cavity and gestational sac located in the interstitial portion of the tube, $>1 \mathrm{~cm}$ far away from cavity $[4,6,29]$.

Conservative management may be appropriate in patients who are hemodynamically stable without rupture and with a low or falling beta hCG but carries a risk 


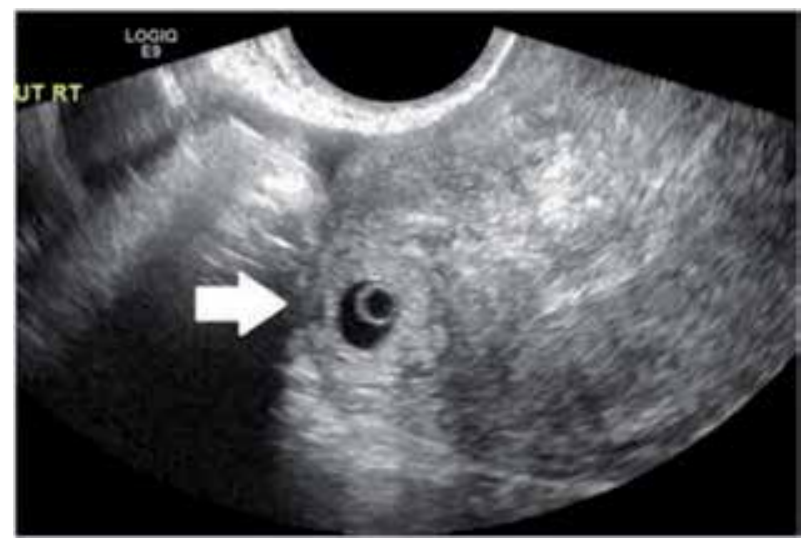

Figure 2.

Empty uterine cavity and gestational sac located in the interstitial portion of the tube, $>1 \mathrm{~cm}$ far away from cavity [27].

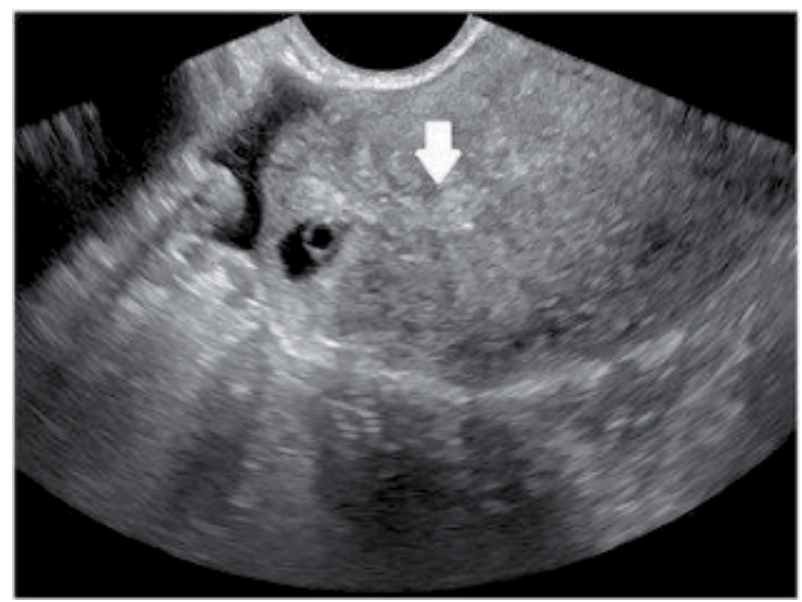

Figure 3.

"Interstitial line" [27].

of uterine rupture due to the weakened myometrial wall [4]. Medical management is to use of methotrexate either given systemically or by local injections. Single- or multiple-dose methotrexate regimens have success rates between 66 and 100\% [9]. There is risk of failure due to increased vascularity, higher beta hCGs, and larger gestational sacs, so patient selection must be critical. Using systemic methotrexate in combination with gefitinib (oral epidermal growth factor receptor inhibitor) may be an alternative therapy [30].

Surgical treatment is indicated when medical management failure, according to patient preference, or if there is hemodynamic instability, severe hemorrhage, and/ or findings concerning rupture, including pain or imaging evidence of hemoperitoneum. Minimally invasive surgeries are cornuostomy, salpingostomy, and cornual resection for earlier diagnosis. Cornuostomy (entails a linear incision, following the injection of dilute vasopressin at the cornua to minimize blood loss), cornual resection, or salpingostomy is being used for smaller interstitial ectopic pregnancies measuring $<3.5 \mathrm{~cm}[31,32]$. Cornual resection has been recommended for advanced management of more interstitial pregnancies $>3.5 \mathrm{~cm}[33,34]$. Laparotomy and hysterectomy are still the first-line treatment in patients with hemodynamic instability and severe hemorrhage. Selective uterine artery embolization can be used in 
conjunction with methotrexate in order to reduce hemorrhage, but there are concerns about the safety and complications of future pregnancies after this technique $[35,36]$.

\section{Cornual ectopic pregnancy}

Cornual pregnancies are one of the rare forms of ectopic pregnancy at $0.2-2 \%$ and occur in a cornua of a bicornuate uterus, in an rudimentary horn, in a unicornuate uterus, and/or in a septate uterus [37]. Cornual and interstitial pregnancies are often referred to interchangeably, but the following criteria can be used to diagnose cornual pregnancy on ultrasound examination [38]:

1. A single interstitial portion of fallopian tube in the main uterine body.

2. A mobile gestational sac surrounded by myometrium and separate from the uterus.

3. Gestational sac adjoining to the unicornuate uterus with a vascular pedicle.

Methotrexate is generally ineffective due to late diagnosis. Surgery is the main management for cornual ectopic pregnancies that includes myomectomy for an unruptured ectopic, laparoscopic cornuotomy, cornual resection, or excision of the rudimentary horn $[4,39-41]$. Laparotomy and hysterectomy may prove necessary due to hemorrhage or large cornual ectopics. Elective Cesarean section is widely recommended in subsequent pregnancies because of risk of uterine rupture.

\section{Ovarian ectopic pregnancy}

Ovarian ectopic pregnancy accounts for 3\% of all ectopic pregnancies [42]. Previous pelvic inflammatory disease, endometriosis, and assisted reproductive technologies seem as risk factors [43-47]. Interference in the release of the ovum from the ruptured follicle, tubal malfunction, and inflammatory thickening of the tunica albuginea are suggested etiologies [48]; however, ovarian ectopic pregnancies have been reported in patients lacking fallopian tubes [49]. Usually symptoms present with abdominal pain and light vaginal spotting. Diagnosis can be difficult to differentiate from a hemorrhagic or corpus luteal cyst or indeed a tubal ectopic.

Diagnostic criteria described by Spiegelberg [50] (Figure 4):

1. Completely intact fallopian tubes.

2. Anatomically gestational sac located in the normal position at the ovary.

3. Both ovary and gestational sac connected to the uterus by ovarian ligament.

4. Placental trophoblastic tissue attached to the ovarian cortex.

Also ovarian EPs may be suspected by ultrasound when a hypoechogenic area is seen with peripheral Doppler flow surrounded by a wide echogenic ring and may be completely surrounded by ovarian cortex, and a fetal pole is rarely present $[38,43]$.

Management is most commonly surgical, and little data is available on the medical management of this condition with systemic MTX either single- or 


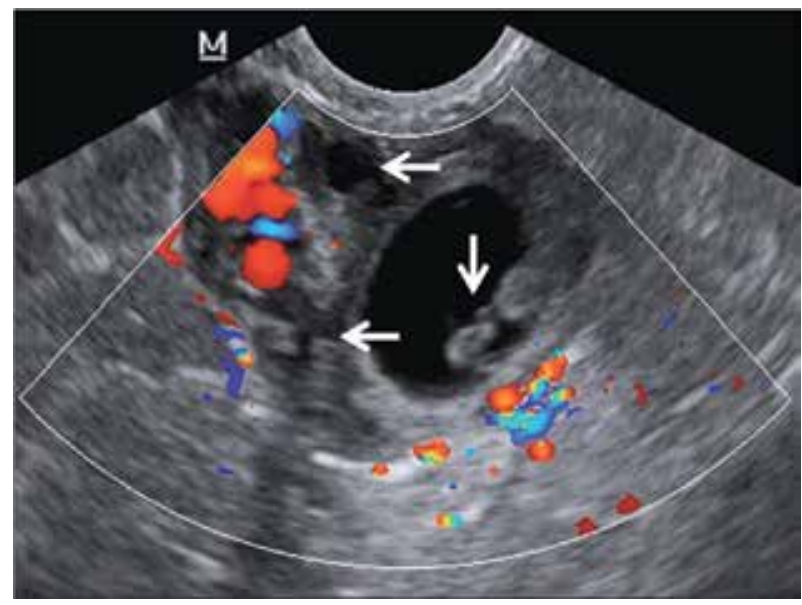

Figure 4.

Ovarian ectopic pregnancy [51].

multiple-dose regimens [2]. Fifty milligrams of MTX injections directly into the ovarian EP with transvaginal or laparoscopically have also been reported as a successful management $[52,53]$. MTX may also be used in the treatment of persistent trophoblastic tissue after laparoscopy [45]. Partial or total oophorectomy or ovarian wedge resection with laparoscopic surgery has become the standard for management of hemodynamically stable patients $[7,9]$. Conservative resection (wedge resection) is performed in patients who want to preserve their fertility.

\section{Abdominal ectopic pregnancy}

The rarest form of ectopic pregnancies is at $0.9-1.4 \%$ and relates to implantation at sites throughout the abdomen including omentum; organs such as the liver, spleen, and bowel; large vessels; pelvic cul-de-sac; broad ligament; and pelvic side wall $[4,28,54-56]$. Abdominal ectopic pregnancy can be defined as primary or secondary; when the fimbrial end does not 'pick up' the ovulated follicle is primary type, tubal abortion via the fimbria and peritoneal implantation related with secondary abdominal ectopics [4, 28]. Risk factors are similar to tubal ectopic prior history of a medically treated ectopic, previous pelvic inflammatory disease, prior surgery, endometriosis, and assisted conception. Abdominal ectopic pregnancies have been described after ART, specifically after IUI [57], after IVF [58], and after Clomid [59]. Symptoms include abdominal pain, painful fetal movements, vaginal bleeding, nausea, and vomiting. Abdominal X-ray, ultrasound, or diagnostic laparoscopy are used to diagnose, although MRI may be beneficial. İn several case reports, diagnosis is only made at Cesarean section [60].

Diagnostic ultrasound criteria have been suggested by Gerli et al. [61]:

1. Absence of an intrauterine gestation sac.

2. Absence of tube and a complex adnexal mass.

3. Gestational cavity surrounded by loops of bowel and separated by peritoneum.

4. Wide mobility similar to fluctuation of the sac with pressure of the transvaginal probe toward the posterior cul-de-sac. 
Laparotomy and delivery with removal of the fetus with or without placental tissue are the traditional management [62]. The maternal mortality rate is eight times higher than for any other ectopic pregnancies $[63,64]$. Expectant management suggested at rare reports orders to attain a live birth. If abdominal pregnancy is diagnosed after the twentieth week of gestation, expectant management can be considered with close follow-up at a tertiary health care facility. Delivery is recommended at 34 weeks if fetus has no congenital malformations, and placenta which implanted away from upper abdomen is often left in place to avoid the risk for hemorrhage [65, 66]. A few case reports have described subsequent methotrexate treatment and ultrasoundguided injection of potassium chloride [4] or radiological artery embolization to minimize blood loss before leaving placental tissue behind [28, 56, 67-69].

\section{Cesarean scar pregnancy}

Cesarean scar pregnancies are extremely rare, 1:2226 of all pregnancies and $6 \%$ of all ectopic pregnancies in women who have undergone at least one previous Cesarean section [4]. Scar pregnancies may be presented with painless vaginal bleeding and also associated with significant rates of uterine rupture and major hemorrhage, uterine rupture, and hypovolemic shock [50]. Risk factors include previous Cesarean, myomectomy, dilatation and curettage, adenomyosis, IVF, and manual removal of the placenta $[28,70]$. The suggested theory of pathogenesis is the blastocyst enters a microscopic tract in the uterine scar and implants in the deficient uterine wall. The impact of the number of previous Cesarean sections, the time interval between Cesarean sections, and the rate of scar implantation are unclear [71-73]. With increasing rates of Cesarean section and repeated Cesarean sections, the scar surface area is getting bigger and is increasingly deficient due to fibrosis, poor vascularity, and postoperative healing, thereby leading to higher rates of blastocyst implantation [72]. Although it is suggested to use ultrasound with Doppler, hysteroscopy, and MRI to diagnose and differentiate Cesarean scar from cervical ectopic pregnancies, transvaginal ultrasound is the first-line approach [50].

Ultrasound diagnostic criteria by Jurkovic et al. [74]:

1. An empty uterine cavity without contact with the sac.

2. Gestational sac located anteriorly at the level of the internal os covering the visible or presumed site of the previous lower uterine segment of the prior hysterotomy (Figure 5).

3. The myometrium must be very thin (1-3 mm) or absent between the bladder and sac (Figure 6).

4. A negative "sliding organ sign" and the presence of peripheral Doppler flow.

There is no definitive consensus of treatment, yet first-trimester termination is recommended to prevent uterine rupture, major hemorrhage, life-threatening complications, and maternal morbidity and preserve future fertility. Term births are associated with hemorrhage and emergent cesarean hysterectomy [74, 77, 78]. Systemic methotrexate can be used in hemodynamically stable patients with an unruptured scar pregnancy, $<8$ gestation weeks, and a myometrial thickness of $<2 \mathrm{~mm}$ between the pregnancy and the bladder and more successful if beta hCG level is $<5000 \mathrm{IU} / \mathrm{L}$ [74]. Local potassium chloride, bilateral uterine artery injection of methotrexate 


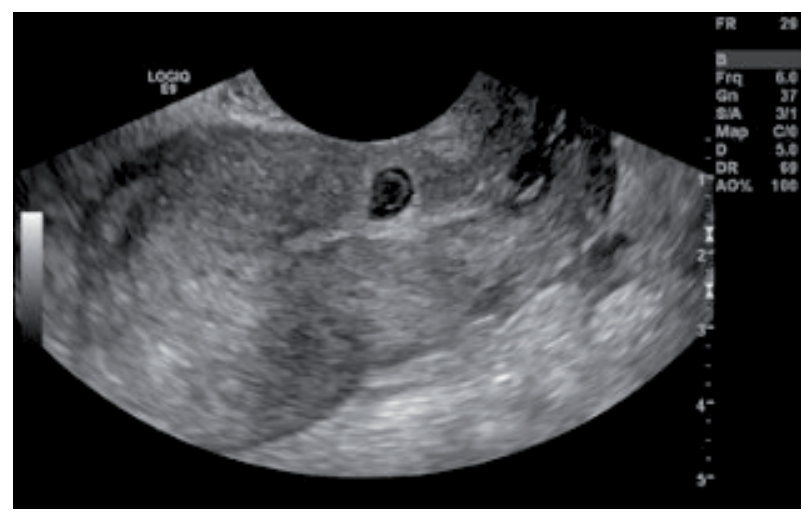

Figure 5.

Caesarean scar pregnancy [75].

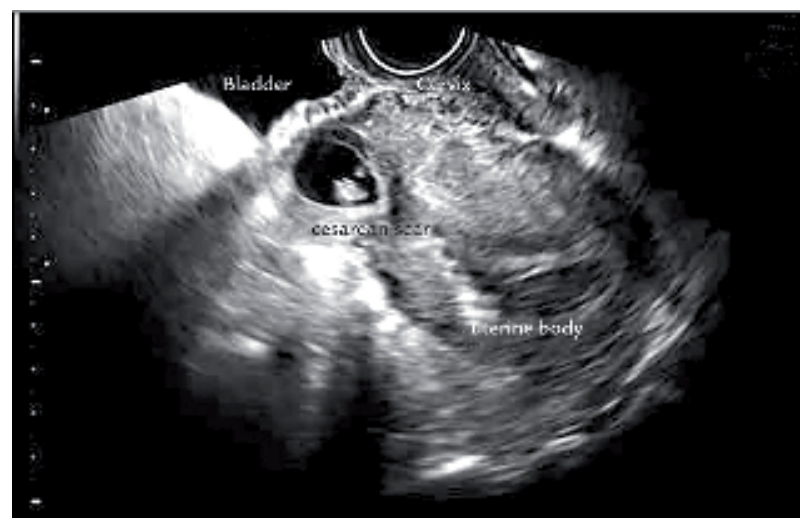

Figure 6.

Myometrial thickness of scar pregnancy [76].

combined with embolization, and combination of gefitinib and systemic methotrexate have been described $[4,30]$. Surgical approaches are uterine curettage, resection or excision with hysteroscopy, laparoscopy or laparotomy. Curettage is an accepted treatment under ultrasound guidance following chemotherapy, but it should not be performed as a first-line treatment because of complication with hemorrhage. Resection allows for revision of the lower uterine segment, which theoretically may reduce risk for recurrence [79]. Hysteroscopic resection is not recommended when the residual myometrium is less than $3 \mathrm{~mm}$, given the risk of anterior wall perforation and bladder injury $[80,81]$. Hysterectomy may be required for uterine rupture or more advanced pregnancies [4].

\section{Conclusion}

Non-tubal ectopic pregnancies are rare but can be a life-threatening condition due to late diagnosis. Earlier diagnosis and treatment of patients are associated with decreased morbidity and mortality in non-tubal pregnancy and, very importantly, preserve the uterus and subsequent fertility [28]. Clinicians should have a high index of suspicion in patients presenting with pain and bleeding in pregnancy and take careful note of their previous obstetric and gynecology history to identify key risk factors for ectopic pregnancy. Ultrasound criteria now exist for all non-tubal 
ectopic pregnancies, facilitating early diagnosis and giving the patient options for management. It seems reasonable therefore to treat these pregnancies with a combination of local or systemic chemotherapy and/or surgical removal. Increased experiences have led to choose the best way to manage non-tubal pregnancies and develop new techniques.

\section{Author details}

Aliye Nigar Serin ${ }^{1 *}$ and Özer Birge ${ }^{2}$

1 Department of Gynaecology and Obstetrics, Osmaniye State Hospital, Osmaniye, Turkey

2 Department of Gynaecology and Obstetrics, Akdeniz University Hospital, Antalya, Turkey

*Address all correspondence to: nserin85@hotmail.com

\section{IntechOpen}

(C) 2019 The Author(s). Licensee IntechOpen. This chapter is distributed under the terms of the Creative Commons Attribution License (http://creativecommons.org/licenses/ by/3.0), which permits unrestricted use, distribution, and reproduction in any medium, provided the original work is properly cited. (cc) BY 


\section{References}

[1] Barnhart K. Ectopic pregnancy. The New England Journal of Medicine. 2009;361:379-387

[2] Practice Committee of the American Society for Reproductive Medicine. Medical treatment of ectopic pregnancy: A committee opinion. Fertility and Sterility. 2013;100:638-644

[3] Cecchino GN, Junior EA. Methotrexate for ectopic pregnancy: When and how. Archives of Gynecology and Obstetrics. 2014;290:417-423

[4] Shen L, Fu J, Huang W, Zhu H, Wang Q, Yang S, et al. Interventions for non-tubal ectopic pregnancy. Cochrane Database of Systematic Reviews. 2014;7:CD011174

[5] Al-Memar M, Kirk E, Bourne T. The role of ultrasonography in the diagnosis and management of earl pregnancy complications. The Obstetrician and Gynaecologist. 2015;17:173-181

[6] Kirk E, Bottomley C, Bourne T. Diagnosing ectopic pregnancy and current concepts in the management of pregnancy of unknown location. Human Reproduction Update. 2014;20(2):250-261

[7] Taran FA, Kagan KO, Hubner M, Hoopman M, Wallwiener D, Bricker S. The diagnosis and treatment of ectopic pregnancy. Deutsches Ärzteblatt International. 2015;112:693-704

[8] Tong S, Sjubisz M, Horne AW. Molecular diagnostics and therapeutics for ectopic pregnancy. Molecular Human Reproduction.

2015;21(2):126-113

[9] Panelli D, Phillips CH, Brady PC. Incidence, diagnosis and management of tubal and nontubal ectopic pregnancies: A review. Fertility Research and Practice. 2015;1:15
[10] Benson CB, Doubilet PM. Strategies for conservative treatment of cervical ectopic pregnancy. Ultrasound in Obstetrics and Gynecology. 1996;8:371-372

[11] Cepni I, Ocal P, Erkan S, Erzik B. Conservative treatment of cervical ectopic pregnancy with transvaginal ultrasound guided aspiration and single-dose methotrexate. Fertility and Sterility. 2004;81(4):1130-1132

[12] Ushakov FB, Elchalal U, Aceman PJ, Schenker JG. Cervical pregnancy:

Past and future. Obstetrical and Gynecological Survey. 1997;52:45-59

[13] Varghese U, Fajardi A, Gomathinayagam T. Cervical pregnancy. 2007. Available from: http://www.omjournal.org/ BriefCommunication/Full text/200801/ cervical\%20Pregnancy.html

[14] Pisarska MD, Carson SA. Incidence and risk factors for ectopic pregnancy. Clinical Obstetrics and Gynecology. 1999;42:2-8

[15] Bennett S, Waterstone J, Parsons J, Creighton S. Two cases of cervical pregnancy following in vitro fertilization and embryo transfer to the lower uterine cavity. Journal of Assisted Reproduction and Genetics. 1993;10:100-103

[16] Leeman LM, Wendland CL. Cervical ectopic pregnancy. Diagnosis with endovaginal ultrasound examination and successful treatment with methotrexate. Archives of Family Medicine. 2000;9(1):72-77

[17] Raskin MM. Diagnosis of cervical pregnancy by ultrasound: A case report. American Journal of Obstetrics and Gynecology. 1978;130(2):234-235

[18] Samal SK, Rathod S. Cervical ectopic pregnancy. Journal of Natural 
Science, Biology and Medicine. 2015;6(1):257-260

[19] Hofmann HM, Urdl W, Höfler H, Hönigl W, Tamussino K. Cervical pregnancy: Case reports and current concepts in diagnosis and treatment. Archives of Gynecology and Obstetrics. 1987;241:63-69

[20] Jurkovic D, Hacket E, Campbell S. Diagnosis and treatment of early cervical pregnancy. A review and a report of two cases treated conservatively. Ultrasound in Obstetrics and Gynecology. 1996;8:373-380

[21] Kung FT, Chang SY. Efficacy of methotrexate treatment in viable and non viable cervical pregnancies. American Journal of Obstetrics and Gynecology. 1999;181(6):1438-1444

[22] Spitzer D, Steiner H, Graf A, et al. Conservative treatment of cervical pregnancy by curettage and local prostaglandin injection. Human Reproduction. 1997;12(4):860-866

[23] Hung TH, Shau WY, Hsieh TT, Hsu JJ, Soong YK, Jeng CJ. Prognostic factors for an unsatisfactory primary methotrexate treatment of cervical pregnancy: A quantitative review. Human Reproduction. 1998;13(9):2636-2642

[24] Júnior JE, Musiello RB, Araujo Júnior E, Souza E, Fava JL, Guerzet EA, et al. Conservative management of cervical pregnancy with embryonic heart activity by ultrasound-guided local injection: An eight case series. The Journal of Maternal-Fetal and Neonatal Medicine. 2014;27(13):1378-1381

[25] Murji A, Garbedian K, Thomas J, Cruickshank B. Conservative management of cervical ectopic pregnancy. Journal of Obstetrics and Gynaecology. 2015;37(11):1016-1020

[26] Lau S, Tulandi T. Conservative medical and surgical management of interstitial ectopic pregnancy. Fertility and Sterility. 1999;72:207-215

[27] Ackerman TE, Levi CS, Dashefsky SM, Holt SC, Lindsay DJ. Interstitial line: Sonographic finding in interstitial (cornual) ectopic pregnancy. Radiology. 1993;189(1):83-87

[28] Chetty M, Elson J. Treating nontubal ectopic pregnancy. Best Practice and Research. Clinical Obstetrics and Gynaecology. 2009;23:529-538

[29] Casikar I, Condous G. How to effectively diagnose ectopic pregnancy on ultrasound. Expert Review Obstetrics Gynecology. 2013;8(6):493-495

[30] Horne AW, Skubisz MM, Tong S, Duncan WC, Neil P, Wallace EM, et al. Combination gefitinib and methotrexate treatment for non-tubal ectopic pregnancies: A case series. Human Reproduction. 2014;29(7):1375-1379

[31] Pasic R, Wolfe WM. Laparoscopic diagnosis and treatment of interstitial ectopic pregnancy: A case report. American Journal of Obstetrics and Gynecology. 1990;163:587-588

[32] Tulandi T, Vilos G, Gomel V. Laparoscopic treatment of interstitial pregnancy. Obstetrics and Gynecology. 1995;85:465-467

[33] Ng S, Hamontri S, Chua I, Chern B, Siow A. Laparoscopic management of 53 cases of cornual ectopic pregnancy. Fertility and Sterility. 2009;92:448-452

[34] Grobman WA, Milad MP.

Conservative laparoscopic management of a large cornual ectopic pregnancy. Human Reproduction. 1998;13:2002-2004

[35] Deruelle P, Lucot J-P, Lions C, Robert Y. Management of interstitial pregnancy using selective uterine 
embolisation. Obstetrics and

Gynecology. 2005;106:1165-1167

[36] Ophir E, Singer-Jordan J, Oettinger $\mathrm{M}$, et al. Uterine artery embolization for management of interstitial twin ectopic pregnancy: Case report. Human Reproduction. 2004;19:1774-1777

[37] Nahum GG. Rudimentary uterine horn pregnancy. The 20th century worldwide experience of 588 cases. The Journal of Reproductive Medicine. 2002;47:151-163

[38] Jurkovic D, Marvelos D. Catch me if you can: Ultrasound diagnosis of ectopic pregnancy. Ultrasound in Obstetrics and Gynecology. 2007;30:1-7

[39] Walid MS, Heaton RL. Diagnosis and laparoscopic treatment of cornual ectopic pregnancy. German Medical Science. 2010;8:16. DOI: 10.3205/000105

[40] Alalade A, Mayers K, Abdulrahman G, Oliver R, Odejinmi F. A 12 year analysis of nontubal ectopic pregnancies: Do the clinical manifestations and risk factor for these rare pregnancies differ from those of tubal pregnancies? Gynecological Surgery. May 2016;13(2):103-109

[41] Kulkarni K, Ajmera S. Pregnancy in rudimentary horn of uterus. Indian Journal of Medical Sciences. 2013;67(1-2):45-47

[42] Bouyer J, Coste J, Ferrnandez H, et al. Sites of ectopic pregnancy: A 10 year population-based study of 1800 cases. Human Reproduction. 2002;17:3224-3230

[43] Comstock C, Huston K, Lee W. The ultrasonographic appearance of ovarian ectopic pregnancies. Obstetrics and Gynecology. 2005;105(1):42-46
[44] Bontis J, Grimbizis G, Tarlatzis BC, et al. Intrafollicular ovarian pregnancy after ovulation induction/intrauterine insemination: Pathophysiological aspects and diagnostic problems. Human Reproduction. 1997;12(2):376-378

[45] Einenkel J, Baier D, Horn L-C, et al. Laparoscopic therapy of an intact primary ovarian pregnancy with ovarian hyperstimulation syndrome. Human Reproduction. 2000;15(9):2037-2040

[46] Ghi T, Banfi A, Marconi R, et al. Three-dimensional sonographic diagnosis of ovarian pregnancy. Ultrasound in Obstetrics and Gynecology. 2005;26:102-104

[47] Marret H, Hamamah S, Alonso AM, et al. Case report and review of the literature: Primary twin ovarian pregnancy. Human Reproduction. 1997;12(8):1813-1815

[48] Panda S, Darlong LM, Singh S, et al. Case report of a primary ovarian pregnancy in a primigravida. Journal of Human Reproductive Sciences. 2009;2(2):90-92

[49] Hsu CC, Yang TT, Hsu CT. Ovarian pregnancy resulting from cornual fistulae in a woman who had undergone bilateral salpingectomy. Fertility and Sterility. 2005;83(1):205-207

[50] Molinaro TA, Barnhart KT. Ectopic pregnancies in unusual locations. Seminars in Reproductive Medicine. 2007;25(2):123-130

[51] Kachewar SG, Sankaye SB. Ovarian ectopic pregnancy: A case report. Journal of Mahatma Gandhi Institute of Medical Sciences. 2016;21(2):147-150

[52] Mittal S, Dadhwal V, Baurasi P. Successful medical management of ovarian pregnancy. International Journal of Gynaecology and Obstetrics. 2003;80:309-310 
[53] Pagidas K, Frishman GN.

Nonsurgical management of primary ovarian pregnancy with transvaginal ultrasound-guided local administration of methotrexate. Journal of Minimally Invasive Gynecology. 2013;20:252-254

[54] Dover RW, Powell MC. Management of a primary abdominal pregnancy. American Journal of Obstetrics and Gynecology. 1995;172:1603-1604

[55] Ludwig M, Kaisi M, Bauer $\mathrm{O}$, et al. The forgotten child-A case of heterotopic, intra-abdominal and intrauterine pregnancy carried to term. Human Reproduction. 1999;14(5):1372-1374

[56] Varma R, Mascarenhas R, Jame D. Successful outcome of advanced abdominal pregnancy with exclusive omental insertion. Ultrasound in Obstetrics and Gynecology. 2003;21:192-194

[57] Kar S. Primary abdominal pregnancy following intra-uterine insemination. Journal of Human Reproductive Sciences. 2011;4(2):95-99

[58] Koyama S, et al. A case of abdominal pregnancy following in vitro fertilization and embryo transfer treated with laparoscopic surgery. Gynecology and Minimally Invasive Therapy. August 2016;5(3):132-133. DOI: 10.1016/j. gmit.2015.04.006

[59] Baba T, Endo T, Ikeda K, Takenami N, Shimizu A, Morishita M, et al. Simultaneous presentation of tubal and primary abdominal pregnancies following clomiphene citrate treatment. Archives of Gynecology and Obstetrics. 2012;286(2):395-398

[60] Tromans PM, Coulson R, Lobb M. Abdominal pregnancy associated with extremely elevated serum alphafetoprotein: Case report. British Journal of Obstetrics and Gynaecology. 1984;91:296-298
[61] Gerli S, Rossetti D, Baiocchi G, et al. Early ultrasonographic diagnosis and laparoscopic treatment of abdominal pregnancy. European Journal of Obstetrics, Gynecology, and Reproductive Biology. 2004;113:103-105

[62] Ayinde OA, Aimakhu CO, Adeyanju OA, et al. Abdominal pregnancy at the university college hospital, Ibadan: A ten-year review. African Journal of Reproductive Health. 2005;9:123-127

[63] Atrash HK, Friede A, Hogue CJ. Abdominal pregnancy in the United States: Frequency and maternal mortality. Obstetrics and Gynecology. 1987;69(3 Pt 1):333-337

[64] Tulandi T, Saleh A. Surgical management of ectopic pregnancy. Clinical Obstetrics and Gynecology. 1999;42(1):31-38

[65] Beddock R, Naepels P, Gondry C, Besserve P, Camier B, Boulanger JC, et al. Diagnosis and current concepts of management of advanced abdominal pregnancy. Gynécologie, Obstétrique and Fertilité. 2004;32:55

[66] Hreshchyshyn M, Bogen B, Loughran C. What is the actual presentday management of the placenta in late abdominal pregnancies? American Journal of Obstetrics and Gynecology. 1961;81:302-317

[67] Cardosi RJ, Nackley AC, Londono J, Hoffman M. Embolization for advanced abdominal pregnancy. The Journal of Reproductive Medicine. 2002;47(10):861-863

[68] Jazayeri A, Davis TA, Contreras DN. Diagnosis and management of abdominal pregnancy. A case report. The Journal of Reproductive Medicine. 2002;47(12):1047-1049 
[69] Rahaman J, Berkowitz R, Mitty H, Gaddipati S, Brown B, Nezhat F. Minimally invasive management of an advanced abdominal pregnancy. Obstetrics and Gynecology. 2004;103:1064-1068

[70] Ash A, Smith A, Maxwell D. Caesarean scar pregnancy. British Journal of Obstetrics and Gynaecology. 2007;114(3):253-263

[71] Jin H, Zhou J, Yu Y, et al. Intramural pregnancy: A report of two cases. The Journal of Reproductive Medicine. 2004;49(7):569-572

[72] Kirk E, Bourne T. Ectopic pregnancy. Obstetrics, Gynaecology and Reproductive Medicine. 2011;21(7):207-211

[73] Seow KM, Huang LW, Lin YH, Lin MY, Tsai YL, Hwang JL. Cesarean scar pregnancy: Issues in management. Ultrasound in Obstetrics and Gynecology. 2004;23(3):247-253

[74] Jurkovic D, Hillaby K, Woelfer B, et al. First-trimester diagnosis and management of pregnancies implanted into the lower uterine segment cesarean section scar. Ultrasound in Obstetrics and Gynecology. 2003;21:220-227

[75] Kim DJ, Welch M, Kendall JL. A case of cesarean scar ectopic: A rare but important form of ectopic pregnancy. Critical Ultrasound Journal. 2011;3(1):55-57

[76] de Freitas Leite J, Fraietta R, Júnior JE. Local management with methotrexate of cesarean scar ectopic pregnancy with live embryo guided by transvaginal ultrasound: A case report. Revista da Associação Médica Brasileira. 2016;62(2):184-187

[77] Michaels AY, Washburn EE, Pocius KD, Benson CB, Doubilet PM, Carusi DA. Outcome of cesarean scar pregnancies diagnosed sonographically in the first trimester. Journal of Ultrasound in Medicine. 2015;34:595-599

[78] Herman A, Weinraub Z, Avrech O, Mon R, Ron-El R, Bukovsky Y. Follow up and outcome of isthmic pregnancy located in a previous caesarean section scar. British Journal of Obstetrics and Gynaecology. 1995;102:839-841

[79] Siedhoff MT, Schiff LD, Moulder JK, Toubia T, Ivester T. Roboticassisted laparoscopic removal of cesarean scar ectopic and hysterotomy revision. American Journal of Obstetrics and Gynecology. 2015;212:681

[80] Wang G, Liu X, Bi F, Yin L, Sa R, Wang D, et al. Evaluation of the efficacy of laparoscopic resection for the management of exogenous cesarean scar pregnancy. Fertility and Sterility. 2014;101:1501-1507

[81] Marotta ML, Donnez J, Squifflet J, Jadoul P, Darii N, Donnez O.

Laparoscopic repair of post-cesarean section uterine scar defects diagnosed in nonpregnant women. Journal of Minimally Invasive Gynecology. 2013;20:386-391 
Section 3

\section{Diagnosis and Management}





\title{
Chapter 3
}

\section{Non-tubal Ectopic Pregnancy: Diagnosis and Management}

\author{
Adebayo A. Adeniyi and Christopher A. Enakpene
}

\begin{abstract}
Ectopic pregnancy is the leading cause of maternal mortality in the first trimester, and prompt diagnosis and intervention are essential to ameliorate its associated complications. A majority of ectopic pregnancies are tubal, but extra-tubal pregnancy may pose more challenges in diagnosis and treatment. Early diagnosis of extra-tubal pregnancies requires high index of suspicion using transvaginal ultrasound and at times complemented with the help of magnetic resonance imaging. Similar to tubal pregnancy, extra-tubal ectopic pregnancies can be treated using surgical approach via laparotomy versus laparoscopy, or medical intervention with methotrexate, potassium chloride and most recently, mifepristone and epidermal growth factor inhibitor (gefitinib). For abdominal and ovarian ectopic pregnancies, the best surgical approach is via laparotomy or laparoscopy, while for cervical ectopic pregnancy and cesarean scar pregnancy (CSP), initial medical treatment with methotrexate, then suction curettage under ultrasound guidance, or hysteroscopic resection can suffice. All patients with extra-tubal pregnancy should be well counseled about the associated complications, fertility preserving intervention, and need for prolong monitoring especially those that choose medical therapy.
\end{abstract}

Keywords: cervical, ovarian, abdominal, cesarean section scar, interstitial, pregnancy, medical, surgical, treatment

\section{Introduction}

Ectopic pregnancy is defined as the implantation of a fertilized ovum outside the normal endometrial lining of the uterine cavity, and it accounts for $1-2 \%$ of all pregnancies $[1,2]$. The true incidence of ectopic pregnancy is unknown but it accounts for $18 \%$ of women seen in the emergency room for first trimester vaginal bleeding, or abdominal pain, or both [3]. Moreover, ruptured ectopic pregnancies are responsible for $2.7 \%$ of all pregnancy-related deaths, and it is the leading cause of hemorrhage-related mortality [4]. About $95 \%$ of ectopic pregnancies occur in the oviduct or the Fallopian tube, while the remaining $5 \%$ occur in different locations such as the cervix, ovary, abdominal cavity and previous uterine scar especially cesarean section scar [5]. There have also been reported cases of ectopic pregnancy in unusual locations such as the intrahepatic ectopic pregnancy. 


\section{Etiology and risk factors}

The risk factors for tubal ectopic pregnancy are well known, and this include poorly treated pelvic inflammatory disease (PID) mostly due to chlamydia and gonorrhea, history of ectopic pregnancy, tubal surgeries including tubal sterilization procedure, pelvic surgery and congenital anomalies of the Mullerian duct such as abnormally long fallopian tubes, unicornuate or bicornuate uterus. Other risk factors include infertility, smoking, use of intrauterine contraceptive device (Levonorgestrel impregnated or Copper IUD), and utero exposure to diethylstilbestrol. While the causes of extra-tubal pregnancy are less defined, it is clear that assisted reproductive techniques have contributed significantly to the incidence of all ectopic pregnancies especially the extra-tubal pregnancies [6].

\section{Ovarian pregnancy}

Ovarian ectopic is also a rare variant of extra-tubal ectopic pregnancy. It accounts for about $0.5 \%$ of all ectopic pregnancies [7]. The incidence of ovarian ectopic pregnancy after natural conception ranges from 1 in 2000 to 1 in 60,000 deliveries and accounts for $3 \%$ of all ectopic pregnancies among intrauterine devices users [7]. Most ovarian ectopic pregnancy (OEP) will rupture before the end of the first trimester, but few cases that progressed to term have been reported.

Risk factors for ovarian ectopic pregnancy:

1. Embryo migration related to the presence of certain conditions that cause fallopian tube epithelial damage that alters tubal motility [8].

2. A hindrance in the release of the ovum from the ruptured follicle [9].

3. Inflammatory thickening of the tunica albuginea [10].

4. IUD insertion is the most significant risk factor for primary OEP in 57-90\% of the cases $[8,9]$. This is because IUD provider protection for intrauterine implantation, but it does not prevent ovarian implantation [11].

Criteria for diagnosis of ovarian pregnancy as described by Spiegelberg in 1878:

1. Fallopian tube on the ipsilateral (affected) side must be intact.

2. The gestational sac must occupy the normal anatomical position of the ovary in the ovarian fossa (see Figure 1) [12].

3. The gestational sac is connected to the uterus by the ovarian ligament.

4. Ovarian tissue must be found on the wall of ectopic pregnancy on histological examination [13].

\subsection{Treatment}

The classical management of ovarian pregnancies is surgical. Early bleeding for small lesson has been managed by ovarian wedge section or cystectomy [14]. With larger lesions, ovariectomy is most often performed and this can be performed via laparotomy or laparoscopy which can be used to excise the gestational sac, to perform laser ablation or use of bipolar electrocoagulation. Methotrexate has been used successfully to treat unruptured ovarian ectopic [15]. 


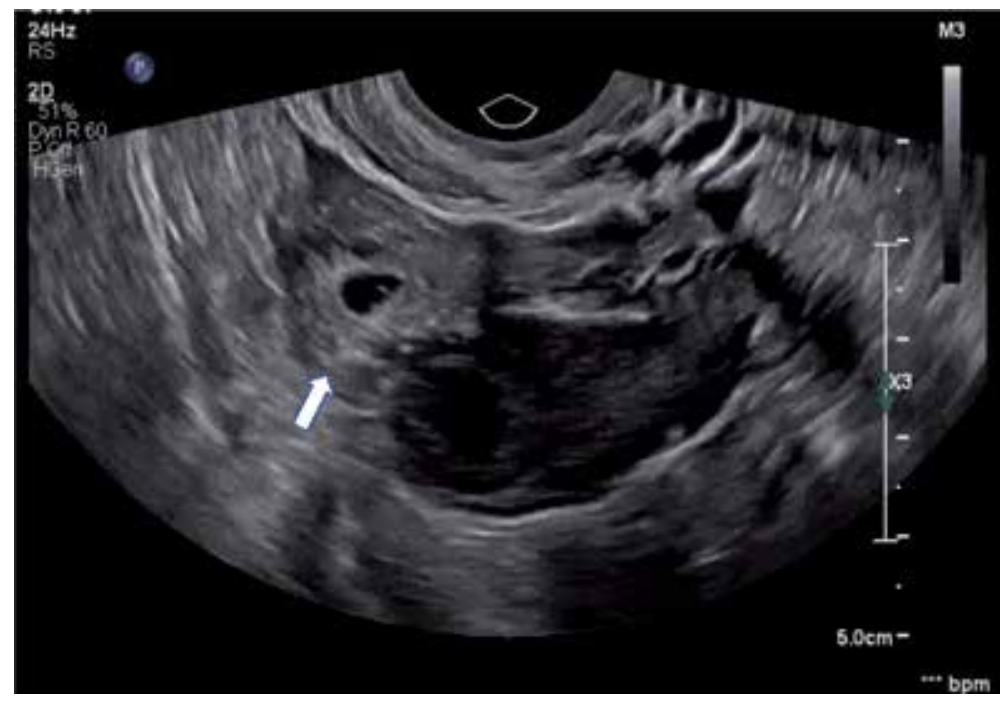

Figure 1.

Ovarian ectopic pregnancy. Transvaginal ultrasound showing a gestational sac located within the ovarian stroma (white arrow) [12].

\section{Abdominal pregnancy}

Abdominal pregnancy is almost always due to secondary implantation, with the primary sites being the tube, ovary or even the uterus. The conceptus result from tubal abortion into the peritoneal cavity or escapes through a rent in the uterine scar [16]. However, there have been reports of rare pseudo-abdominal pregnancies where women have abdominal pregnancy after hysterectomy with intact ovaries. This is most likely to have occurred via a fistula in the vaginal cuff in fertile women following total hysterectomy or via cervical canal following supracervical hysterectomy. The average incidence is above 1 in 3000 pregnancies. With the used of ART, the incidence is found rising [17]. Primary implantation of the fertilized ovum on the peritoneum is so rare that its existence is questionable.

\subsection{Diagnosis}

The diagnostic criteria of abdominal pregnancy as described by Studdiford in 1942:

1. Both the tubes and ovaries are normal without evidence of recent injury.

2. Absence of utero-peritoneal fistula.

3. Presence of a pregnancy related exclusively to the peritoneal surface and early enough to eliminate the possibility of secondary implantation following primary nidation in the tube [18].

Apart from symptoms of lower abdominal pain and amenorrhea, symptoms of normal uterine pregnancy are often exaggerated such as nausea, vomiting, constipation and increased fetal movements. The fetal parts are felt easily and persistent abdominal attitude and position of the fetus on repeated examination is quite common. While abdominal high position the fetus is commonly found in intraperitoneal pregnancy, though the fetus may be lying low in intraligamentary pregnancy. The cervix is not typically soft and is usually displaced 
depending upon the position of the sac. Investigations done in case of suspected abdominal ectopic pregnancy include abdominopelvic ultrasound which shows absence of uterine wall around the fetus with close approximation to maternal abdominal wall and visualization of the uterus separately. Magnetic resonance imaging (MRI) can confirm the diagnosis and may be very accurate. Computed Tomography (CT) is diagnostic and superior to MRI [19]. CT has the risk of radiation. Lateral X-ray on standing position shows superimposition of fetal shadow with the maternal spine shadow.

\subsection{Treatment}

Once the diagnosis is made the opinion is almost crystallized in favor of urgent laparotomy irrespective of period of gestation. The risks of continuation of pregnancy are; catastrophic hemorrhage, fetal death, increased fetal malformation and increased neonatal loss [20]. Thus, continuation of pregnancy for few weeks hoping the baby to become mature enough to survive can only be justified in exceptional circumstances such as a case where the pregnancy continued up to 30 weeks and eventually resulted in a live born baby [21]. The patient and her relatives should be informed about the eventuality. During the period, the patient should be in the hospital. The ideal surgery is to remove the entire sac, fetus, the placenta and the membranes. This may be achieved if the placenta is attached to a removable organ like uterus or broad ligament. However, if the placenta is attached to vital organs, it is better to take the fetus and leave behind the placenta and the sac after tying and cutting the umbilical cord close to the placental attachment. In such a situation, placental activity is to be monitored by quantitative serum $\beta$ HCG level and ultrasound. Complete resorption of the placenta occurs through aseptic autolysis. Complications include secondary hemorrhage, intestinal obstruction and infection.

\section{Other types of non-tubal pregnancy}

The most notable of other extra-tubal ectopic pregnancy are cervical pregnancy, cesarean section scar pregnancy (CSP), interstitial pregnancy and also a recently reported case of intrahepatic pregnancy.

\section{Cervical pregnancy}

Cervical ectopic pregnancies (CEP) probably the rarest of all ectopic pregnancies and it occurs in about 1:16,000 pregnancies, with implantation occurring in the cervical canal at or below the internal cervical os (see Figures 2 and 3) [7, 12, 22-24]. The etiology and risk factors for cervical ectopic pregnancy are similar to those enumerated above, but previous overzealous uterine curettage with associated Asherman syndrome and in-vitro fertilization are probably the most important risk factors. Previous cesarean section has also been implicated as a possible risk factor.

The condition is commonly confused with cervical abortion. In cervical pregnancy, the bleeding is painless and the uterine body lies above the distended cervix. Intractable bleeding following evacuation or expulsion of the products brings about suspicion. The morbidity and mortality are high because of profuse hemorrhage due to paucity of smooth muscles in the cervix, hence unable to contract to stop this bleeding. 
Non-tubal Ectopic Pregnancy: Diagnosis and Management DOI: http://dx.doi.org/10.5772/intechopen.89783

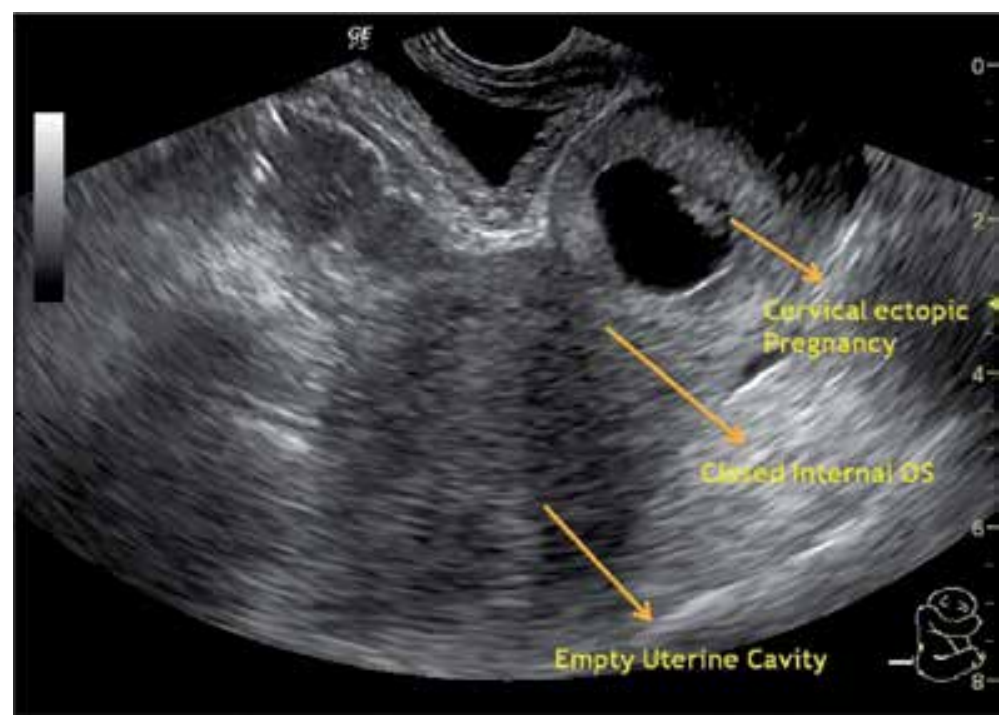

Figure 2.

Early cervical ectopic pregnancy with embryonic pole. Transvaginal ultrasound shows: (1) an empty and normal uterine cavity and (2) a cervical ectopic pregnancy with gestational sac and an embryonic pole (arrows) [23].

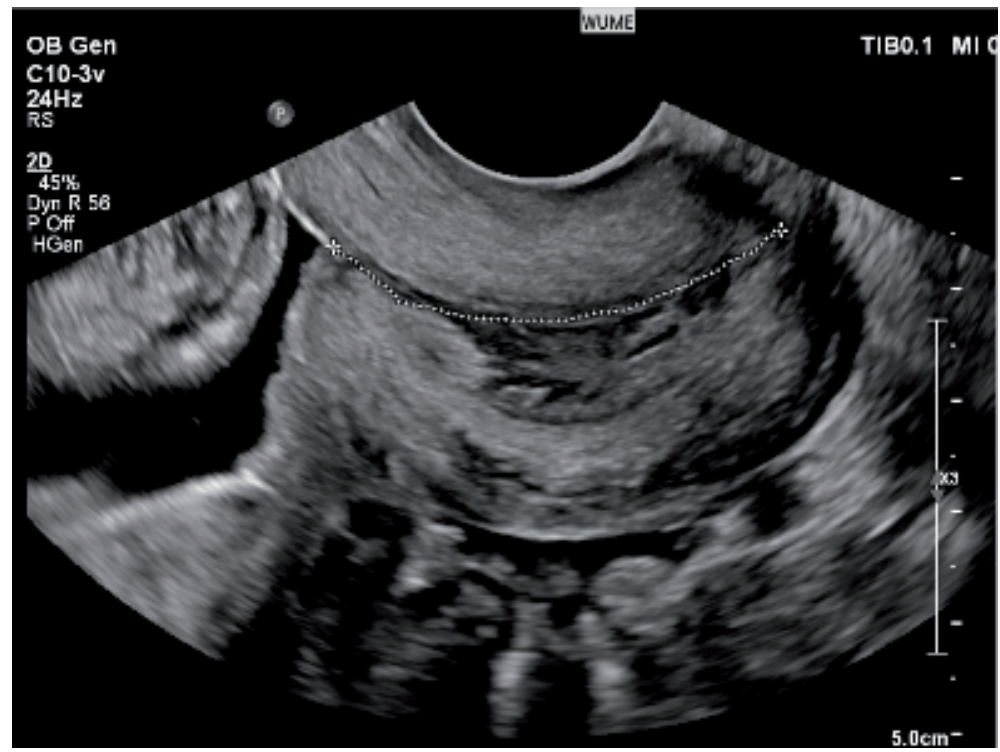

Figure 3.

Cervical ectopic pregnancy with no definite gestational sac. Transvaginal ultrasound showing an organized area with no definite gestational sac located within the posterior cervical stroma (white arrow) [24].

\subsection{Diagnosis}

Many criteria have been proposed for the diagnosis of ectopic pregnancy which include mostly the anatomical and histological criteria described by Rubin in 1911 but later modified in 1983, the more practical criteria by Paalman and McElin and finally, the sonographic criteria by Raskin et al.

The (Rubin, 1983) anatomical and histological criteria for cervical pregnancy include 
1. Cervical gland must be opposite the placental attachment.

2. The placenta attachment to the cervix must be situated below the entrance of the uterine vessels or below the peritoneal reflections of the anterior or posterior surface of the uterus.

3. The fetal elements must be absent from the corpus uteri.

Rubin criteria require a hysterectomy specimen to satisfy these criteria.

Clinical diagnostic criteria as described by Paalman and McElin (1959)

1. Uterine bleeding without cramping pain following a period of amenorrhea.

2. Soft, enlarged cervix equal or larger than the fundus, (the "hourglass" appearance of uterus).

3. Products of conception entirely confined within and firmly attached endocervix.

4. A closed/snug internal cervical os.

5. A partially opened external os.

Raskin (1978) proposed ultrasound diagnostic features but later modified by Timor-Tritsch et al.

1. Cervical enlargement.

2. Uterine enlargement.

3. Diffused intrauterine echoes.

4. Absence of intrauterine pregnancy.

5. Empty uterus or absence of gestational sac or embryo.

6. The entire product of conception (placenta and chorionic villi) must be located below the internal os and the cervical canal must be dilated and barrel shaped.

Jurkovic (1996) criteria were introduced to distinguish primary cervical ectopic pregnancy from an aborting intra-uterine pregnancy:

1. The "sliding sign" seen on transvaginal examination must be absent.

2. The demonstration of peri-trophoblastic blood flow to the fetus by color Doppler flow must be present.

\section{Cesarean scar ectopic pregnancy}

Cesarean scar pregnancy (CSP) accounts for 0.04-0.05 of all pregnancies [1]. The prenatal diagnosis of CSP is by the presence of gestational sac at the site of the previous uterine incision and the presence of an empty uterine cavity and cervix, as well as thin myometrium adjacent to the bladder (see Figure 4) [25, 26]. Cesarean 

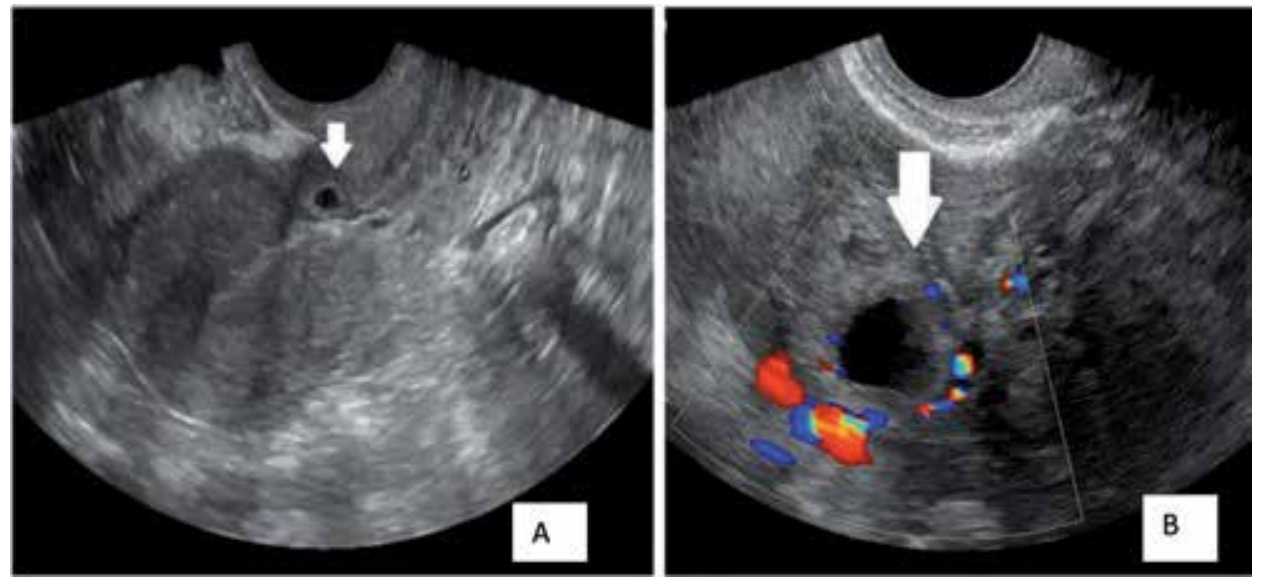

Figure 4.

Cesarean section scar ectopic pregnancy. Transvaginal ultrasound with 2-D and power Doppler showing: (1) normal uterine cavity and endometrium, (2) gestational sac in the cesarean section scar anteriorly, and (3) the vasculature around the gestational sac (white arrows) [25]. (A) shows cesarean ectopic pregnancy on a gray-scale ultrasound, and $(B)$ shows the same pregnancy on color Doppler showing ring of fire of vessels around the gestational sac.

scar pregnancy can be classified based on the degree of invasion of amniotic sac into the myometrium, gestational age at diagnosis, presence of positive fetal heart beats and myometrial thickness of the lower uterine segment. The management of these rarer forms of ectopic pregnancy is just evolving.

Diagnostic criteria of cesarean scar ectopic pregnancy:

1. Empty uterus with clearly visualized endometrium.

\section{Empty cervical canal.}

3. Gestational sac within the anterior portion of the lower uterine segment at the presumed site of the cesarean scar.

4. Thinned or absent myometrium below the gestational sac and bladder $(<5 \mathrm{~mm}$ in two-thirds of cases) [27].

\section{Cornual pregnancy}

One of the most intriguing of ectopic pregnancies is the cornual or interstitial pregnancy, though not strictly extra-tubal pregnancy. It is neither located in the normal endometrial cavity nor in the normal fallopian tubes, but in the proximal portion of the tube and within the musculature of the uterus. The discussion on extra-tubal pregnancy cannot be complete without pointing out the implications of late diagnosis or misdiagnosis of cornual pregnancy; a potentially dangerous ectopic pregnancy. Often times, it can be mistaking for an intrauterine pregnancy thereby continuing till late first trimester, about 10-12 weeks of gestation. When it ruptures, it can result in rapid catastrophic hemorrhage because of the involvement of the well-vascularized muscular myometrium, and can cause significant maternal morbidity or mortality.

Diagnostic criteria of cornual ectopic pregnancy, 1992 [28]:

1. An empty uterine cavity. 
2. A chorionic sac found separated $(>1 \mathrm{~cm}$ ) from the lateral edge of the uterine cavity.

3. A thin $(<5 \mathrm{~mm})$ myometrial layer surrounding the chorionic sac.

\section{Diagnosis of non-tubal ectopic pregnancies}

\subsection{Pregnancy of unknown location (PUL)}

Usually, the absence of intrauterine pregnancy, adnexal mass or abnormally rising serum beta hCG without evidence of intrauterine gestation are not always a confirmation of ectopic pregnancy. In the absence of any ultrasound evidence of visible pregnancy, the terminology of pregnancy of unknown location (PUL) is use. The prevalence of PUL is between 8 and $31 \%$ and it can be influenced by the quality of ultrasonography in the given unit, and the experience of the ultrasonographer [29, 30]. Even when PUL is being suspected, there is usually no place for diagnostic suction and curettage to determine the location of the pregnancy because this will disrupt a potential intrauterine pregnancy. Sometimes, some cases of PUL may evolve into ectopic pregnancy; tubal or non-tubal that may cause life threatening complications.

\subsection{Ectopic pregnancies}

Transvaginal ultrasound (TVUS) is the first line tool and the gold standard diagnostic apparatus of ectopic pregnancy. Ectopic pregnancy is usually diagnosed on TVUS as the presence of a mass with hyperechoic ring around the gestational sac ("bagel sign") or a gestational sac with a fetal pole with or without cardiac activity. The sensitivity and specificity of TVUS in detecting ectopic pregnancy are 90.9 and $99.9 \%$, respectively, with positive and negative predict values of 93.5 and $99.8 \%$, respectively. Another ultrasound feature of ectopic pregnancy is the trilaminar pattern of endometrial thickness with sensitivity of $38 \%$ and specificity of $94 \%$ for detecting ectopic pregnancy [31]. Magnetic resonance imaging is useful as a troubleshooting tool when ultrasound is equivocal or inconclusive before intervention or therapy [27]. Sagittal, coronal, and transverse sections of T1- and T2-weighted magnetic resonance imaging sequences can be used to show the gestational sac embedded in the anterior lower uterine segment, better evaluate pelvic anatomy, improve intra-operative orientation, and assess the possibility of myometrial invasion and bladder involvement [27, 32, 33]. Magnetic resonance imaging can also be used to measure the volume of the lesion so as to help assess whether methotrexate will be indicated and to predicts its success as medical intervention [27].

\section{Management of non-tubal pregnancy}

The management of extra-tubal ectopic pregnancy depends on the location, size of the gestational sac, presence of fetal heart beats and symptoms at presentation. The definitive management of extra-tubal ectopic pregnancy involves surgical extermination of the gestational sac via laparotomy, laparoscopy, hysteroscopy or suctional curettage under ultrasound guidance. Another advantage of surgical extermination is that there is confirmation of ectopic pregnancy by histological evidence of the presence of villi within the stroma of the affected structure.

Preservative procedures such as placement of cerclage; a pulse-string suture or cervical plugging have been proposed as adjunct treatment for cervical ectopic 
pregnancy. Methotrexates therapy either systemic and /or local injections directly into the gestational sac or intracardiac potassium chloride injection have also been used especially when there is associated embryonic cardiac activity. Uterine artery embolization with Gelfoam has also been used successfully to control hemorrhage. However, hysterectomy is often required to control intractable bleeding. Occasionally, there may be no need for intervention due to declining serum beta hCG levels in asymptomatic patients; however, these patients are usually closely monitored to ensure spontaneous resolution of the pregnancy.

\section{Medical management}

\subsection{Methotrexate}

Methotrexate is a chemotherapeutic anti-metabolite that affects rapidly dividing cells, and it is use in the treatment of cancers and connective tissues disorders such as rheumatoid arthritis. The use of Methotrexate in the medical management of ectopic pregnancy has to be thoroughly given serious consideration to avoid inadvertent administration in patient with intrauterine pregnancy. Hence, careful selection of appropriate candidate for Methotrexate treatment to improve success rate is advised. In most instances, Methotrexate should only be given to patients with confirmed or presumed ectopic pregnancy who are hemodynamically stable with an unruptured gestational sac. The success rate is also dependent on the level of serum beta hCG because single-dose systemic Methotrexate had approximately $90 \%$ success rate if the initial serum hCG level is less than $5000 \mathrm{mIU} / \mathrm{ml}$ [34].

The indications and contraindications of Methotrexate should be reviewed before every use. For some extra-tubal pregnancies like CSP and cervical pregnancies, use of Methotrexate may be considered as the first and only option before surgical intervention to minimize catastrophic hemorrhage. The higher the level of serum beta hCG, the more likely the failure rate of using methotrexate in medical management of all ectopic pregnancies. In view of this, some experts have recommended use of two-dose or multiple-dose regimens for such patients if they are not surgical candidates and medical management is warranted. Lipscomb et al. demonstrated that a high serum hCG level is the most important factor associated with failure of treatment with single-dose MTX protocol among women with tubal ectopic pregnancy [35].

Contraindications to methotrexate therapy $[36,37]$.

Absolute contraindications

1.Pregnancy of unknown location of only one evaluation.

2. Peptic ulcer disease.

3. Intrauterine pregnancy.

4. Sensitivity to methotrexate.

5. Breastfeeding.

6. Suspected tubal rupture.

7. Immunodeficiency. 
8. Active pulmonary disease.

9. Unwilling or unable to return for follow up visits.

Relative contraindications

1. Gestational sac $>3.5 \mathrm{~cm}$.

2. Embryonic cardiac motion.

3. Hepatic, renal, or hematologic dysfunction.

4. Serum beta hCG > $5000 \mathrm{mIU} / \mathrm{ml}$.

\subsection{Pre-treatment evaluation}

Blood type, serum hCG (to be used as "Day 1" level).

Safety labs: full blood counts, complete metabolic panel consisting of AST/ALT, $\mathrm{BUN} / \mathrm{Cr}$, also to be repeated prior to additional MTX dosing.

\subsection{Systemic methotrexate treatment protocol}

Systemic methotrexate is usually given as a single-dose or two-dose or fixed multi-dose regimens. The single-dose regimen of $50 \mathrm{mg}$ of methotrexate per body surface area $\left(\mathrm{BSA}, \mathrm{m}^{2}\right.$ ) is usually given only on the first day, then followed by serial monitoring of serum hCG on days 4 and 7 . There is the possibility of repeating the same dose on days 7 and 11 if there is less than 15\% drop in the levels of hCG between days 4 and 7 or between days 7 and 11. The two-dose regimen also uses $50 \mathrm{mg}$ of methotrexate per BSA $\left(\mathrm{m}^{2}\right)$ and it is predetermined to be given on days 1 and 4 , then followed by serial serum hCG levels on days 4 and 7 or 11 . There is also a possibility of repeating the same dose on days 7 and 11 if the drop in serum hCG levels between days 4 and 7 or days 7 and 11 are less than $15 \%$ respectively $[36,37]$.

The fixed multi-dose regimen uses $1 \mathrm{mg}$ of methotrexate per kilogram body weight for a total of four doses given on alternate days; on the first, third, fifth, and seventh days while Folinic acid rescue is given on the second, fourth, sixth, and eighth days. This regimen requires serial serum hCG monitoring on the days of methotrexate injections until there is more than $15 \%$ drop from the previous level. Thereafter, continue weekly to every 4 weeks monitoring until the serum hCG levels is less than $5 \mathrm{mIU} / \mathrm{ml}[36,37]$.

Methotrexate can also be injected locally into the gestational sac or in the fetal intracardiac space to enhance resolution of the pregnancy. This is usually done under ultrasound guidance and it is given in combination with systemic injection in various dosing regimens. Timor-Tritsch et al. suggested using a fixed dose of $75 \mathrm{mg}$ of methotrexate; $25 \mathrm{mg}$ intracardiac injection, $25 \mathrm{mg}$ inside the gestational sac while the remaining $25 \mathrm{mg}$ is given intramuscularly (Table $\mathbf{1}$ ).

\subsection{Adjuvant medical treatments and other interventions}

Give anti-D immunoglobulin injection if patient is rhesus negative.

Advise patient to discontinue folic acid supplementation during MTX treatment.

Avoid pelvic exams and sexual intercourse during treatment period.

Caution patients: to reduce gastrointestinal side-effects, avoid alcohol and nonsteroidal anti- inflammatory drugs (NSAIDs).

Avoid prolonged exposure to sun due to sun hypersensitivity while on MTX. 
Non-tubal Ectopic Pregnancy: Diagnosis and Management

DOI: http://dx.doi.org/10.5772/intechopen.89783

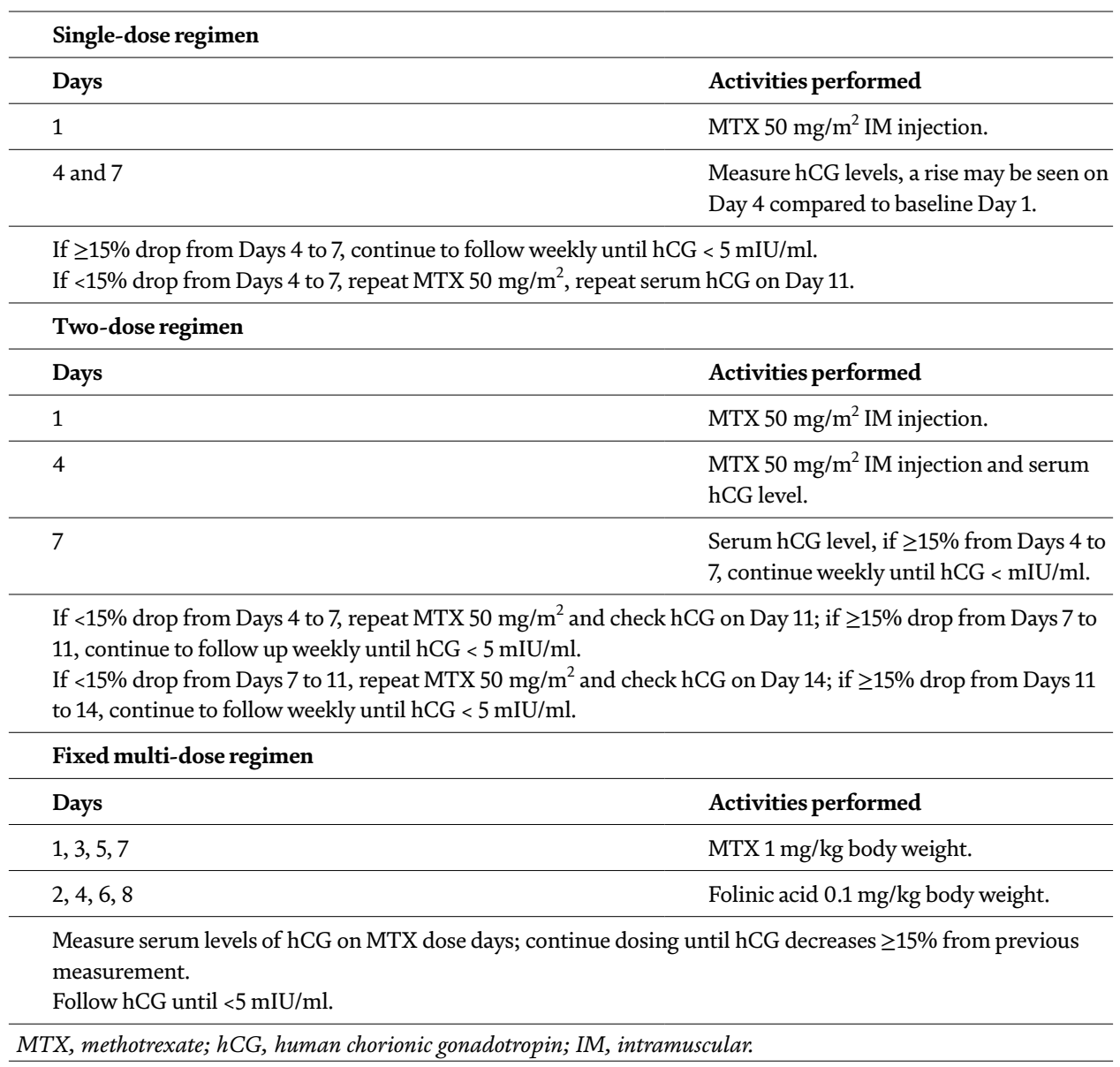

Table 1.

Different regimens of systemic methotrexate.

Avoid new conception until serum hCG is undetectable.

Combination of systemic and local injection of methotrexate.

Timor-Tritsch recommendation of combined local and systemic injection:

A. $25 \mathrm{mg}$ intragestational sac.

B. $25 \mathrm{mg}$ into the placental site as the needle is being withdrawn.

C. $25 \mathrm{mg}$ intramuscular prior to patient discharge from the hospital.

University of Illinois at Chicago protocol (unpublished):

A. Half dose of $50 \mathrm{mg} / \mathrm{m} 2 \mathrm{BSA}$ injected into the intragestational sac.

B. The remaining half dose of MTX is injected intramuscularly.

\subsection{Potassium chloride}

In the presence of cardiac activity, intracardiac injection of potassium chloride with $2 \mathrm{mEq} / \mathrm{L}$ under ultrasound guidance, and repeat until there is no longer 
cardiac activity. It usually requires $2-3 \mathrm{ml}$ injections for the procedure. Potassium chloride can be used as a stand-alone treatment intervention in ectopic pregnancy or in combination with any of the methotrexate injection regimens. In a study of 18 ectopic pregnancies with active embryonic cardiac activity, mean serum beta hCG levels of 33,412 IU/L and mean gestational age of 6 weeks and 6 days, 10 were assigned to KCL injection in the gestational sac while 8 were in the methotrexate group. There was no difference in time to resolution of ectopic pregnancies between those injected with KCL and those with methotrexate [38]. Verma et al. in a threecase series of women with concurrent injection of local KCL and systemic injection of methotrexate, they demonstrated that complete resolution of ectopic pregnancy was achieved and surgery avoided in all 3 cases [39].

\subsection{Newer treatment combinations}

The use of selective progesterone reception modulator (Mifepristone) as an adjuvant for medical therapy has been tried. In a randomized trial by Rozenberg et al., there was no benefit from the systematic addition of mifepristone except in women with progesterone level of $10 \mathrm{ng} / \mathrm{L}$ or more [40]. However, in a larger study involving 72 patients; 30 with combined methotrexate-mifepristone and 42 with methotrexate alone by Perdu et al., the failure rate was lower in the combined group, $1 / 30(33.3 \%)$ compared to $11 / 42(26.2 \%)$ in the methotrexate alone group [41]. Use of epidermal growth factor receptor inhibition in combination with methotrexate has been tried in an in vitro experiment and it showed inhibition of growth of placental cells. These results were confirmed in vivo in mouse models, and it revealed faster rate of fetal resorption when the two drugs were combined [42]. In a phase 1 nonrandomized open label study involving 12 women with ectopic pregnancy and 71 controls, the median level of serum beta hCG by day 7 and the time of pregnancy resolution were significantly lower in the combined methotrexate and epidermal growth factor receptor blocker (gefitinib) compared with methotrexate alone group [43]. Hence, the future goal of medical management of extra-tubal ectopic pregnancies should be combination therapy ab initio with various methotrexate regimen.

\section{Surgical management of non-tubal pregnancy}

To ensure complete removal of a tubal ectopic pregnancy, a British surgeon, Robert Lawson Tait performed a laparotomy with ligation of ruptured tube and board ligament in April 1883. By the 1920s, laparotomy and ligation of the bleeding vessels with removal of the affected tube had become the standard of care, and it remained so until the late 1970s, when operative laparoscopy and salpingectomy replaced laparotomy and salpingectomy. For CSPs, nearly $50 \%$ of clinically diagnosed cases miscarriage during the first trimester but most of them will require additional surgical intervention to stop bleeding [44]. Surgical management of any ectopic pregnancy is associated with a high success rate; low complication rate and short post-treatment follow up [19]. In a national cohort study in the UK by Hart et al. of 102 cases of CSP, the success rates of expectant, medical and surgical management were $43 \%$ (9/21), 46\% (7/15) and 96\% (54/56), respectively. The complication rates were 15/21 (71\%) with expectant, $9 / 15$ (60\%) with medical and 20/56 (36\%) with surgical management. Discharge from care (median number of days) was 82 (range 37-174) with expectant, 21 (range 10-31) with medical and 11 (range 4-49) with surgical management [45]. 


\subsection{Suction curettage}

This surgical intervention has been used to treat appropriately selected suitable patients with cervical ectopic or cesarean scar ectopic pregnancies. It is usually done under transabdominal or transrectal ultrasound guidance to ensure direct visualization of the pregnancy sac during the procedure. This procedure has been used for cervical ectopic pregnancy (CEP) and CSP despite the fear of potential catastrophic hemorrhage that may warrant emergency hysterectomy or may result in significant maternal morbidity and mortality. When suction curettage is being considered as a treatment option, you also need to plan for possible use of adjuvant interventions to reduce excessive blood loss. Adjuvant methods that have used so far include but not limited to intracervical balloon tamponade, angiographic embolization, cervical cerclage, ligation of uterine arteries or local hysteroscopic endocervical resection of the gestational sac with local use of different substances. Among these, arterial embolization has gained more recognition than any other interventions to decrease potentially dangerous massive hemorrhage thought to be associated with suction curettage of CSP or cervical ectopic pregnancy. However, there have been reports of suction curettage used successfully to evacuate CEP or CSP with minimal complications. In a study by Jurkovic et al. of 232 women with CSP, suction curettage was an effective method for the treatment of 191 pregnancies implanted into the lower uterine segment cesarean section scar because of the 116 women who had a follow up visit, only 7 (6.0\%), 95\% CI 1.7-10.3\% required a repeat surgical procedure for retained products of conception. It was associated with a low risk of blood transfusion and hysterectomy. Of the 191 women, $9(4.7 \%)$ required blood transfusion, $95 \%$ CI 1.7-7.7, and 1 (0.5\%) women had life-saving hysterectomy due to uncontrollable intraoperative hemorrhage [46].

\subsection{Wedge resection}

Wedge resection of gestational sac can be used for many extra-tubal pregnancies such as CSP, ovarian or cornual pregnancies. There have been multiple case reports on the safety and effectiveness of wedge resection of CSP via laparotomy. Wedge resection should be considered when the diagnosis is made early, there is no involvement of vital contiguous structures and need for preservation of fertility. In a case report by Vial et al. of 28 years old G3P2002 at 6 weeks gestation, wedge resection of the gestational mass via a Pfannenstiel incision resulted in complete resolution of the pregnancy and subsequent full-term pregnancy delivered by cesarean section [47]. Traditionally, wedge resections of the gestational sac or hysterectomy via laparotomy is the treatment of choice of interstitial or cornual or angular pregnancy. However, laparoscopic cornuotomy is the removal of ectopic pregnancy tissue with preservation of uterine architecture. This increases incidence of persistent and recurrent interstitial pregnancy, but can potentially maintain patient fertility and decrease risk of future uterine rupture. Patients with cornual resection are often delivered at 36-37 weeks of gestation via cesarean section in subsequent pregnancies because of their increased risk of uterine scar rupture. In a study of 29 patients by Liao et al., the incidence of subsequent uterine rupture and dehiscence was 30\% [48]. However, there is still controversy in regards to the recommended surgical technique to treat interstitial pregnancies; cornual resection and cornuectomy are both important considerations.

A small ovarian ectopic pregnancy (OEP) or early bleeding can be treated with wedge resection or cystectomy with the intention to preserve some of the affected ovarian tissues. There have been anecdotal case reports of the 
effectiveness of wedge resection in the treatment of OEP. A case report by Kraemer et al. showed a 29-year-old G1P0 at 8 weeks gestation with confirmed ovarian pregnancy and she was managed by laparoscopic wedge resection of the OEP. She subsequent was pregnant with a normal intrauterine pregnancy 6 months after her surgery [49].

\subsection{Laparotomy/laparoscopy in extra-tubal pregnancy}

Directly visualization of the pregnancy via laparotomy or laparoscopy may be helpful in the definitive diagnosis of OEP and abdominal pregnancy. Surgical management of either of the two pregnancies is influenced by the size of the lesion, the proximity or attachment to vital organs, associated symptoms and the patient's desire for future fertility [46]. Small OEP are usually treated conservatively with wedge resection, enucleation, cystectomy, or trophoblast curettage with electrocoagulation or hemostatic suture to preserve fertility. However, large OEP may require oophorectomy when there are no apparent normal ovarian tissues left [47].

\subsection{Adjuvant surgical interventions}

The use of adjuvant interventions is to accelerate the efficacy of primary treatment or to minimize potential life-threatening complications. Intracervical balloon tamponade or cervical cerclage have been used during cervical curettage for cervical ectopic pregnancy to decrease bleeding. Direct ultrasound-guidance is helpful for all extra-tubal pregnancy to enable injection of methotrexate or KCL or hyperosmolar glucose into the gestational sac. Misoprostol and Methergine are uterotonic agents that cause vasoconstriction, thereby reducing the amount of blood loss during surgical intervention. Careful and appropriate use of these adjuvant treatments can improve successful outcome of primary treatment of extra-tubal pregnancy.

\section{Conclusion}

Ectopic pregnancy is the leading cause of first trimester maternal mortality, and it is mostly located in the fallopian tubes. When it is located outside the tubes, the need for high index of suspicious and prompt intervention is advised because the more advanced extra-tubal pregnancies can result in catastrophic hemorrhage or maternal death. Different surgical techniques that are unique to individual type of ectopic pregnancy, the use of methotrexate and combination therapy have all demonstrated proven benefits for the treatment of extra-tubal pregnancy. 


\section{Author details}

Adebayo A. Adeniyi ${ }^{1,2}$ and Christopher A. Enakpene ${ }^{3 *}$

1 Afe Babalola University, Ado Ekiti, Ekiti State, Nigeria

2 Federal Medical Center, Ido Ekiti, Ekiti State, Nigeria

3 Texas Tech University Health Sciences Center, School of Medicine, Permian Basin, Texas, USA

*Address all correspondence to: christopher.a.enakpene@ttuhsc.edu

\section{IntechOpen}

(C) 2019 The Author(s). Licensee IntechOpen. This chapter is distributed under the terms of the Creative Commons Attribution License (http://creativecommons.org/licenses/ by/3.0), which permits unrestricted use, distribution, and reproduction in any medium, provided the original work is properly cited. (cc) BY 


\section{References}

[1] Rota M, Haberman S, Levgur M. Cesarean scar ectopic pregnancies, etiology, diagnosis and management. Obstetrics and Gynecology. 2006;107:1373-1381

[2] Kwawukume EY, Idrisa A, Ekele BA. Ectopic pregnancy In: Kwawukume EY, Emuveyan EE, editros. Comprehensive Obstetrics in the Tropics 2nd Edition. Accra-North Ghana: Assemblies of God Literature Centre Limited; 2015. pp. 282-287

[3] Barnhart KT, Sammel MD, Gracia CR, Chittams J, Hummel AC, Shaunik A. Risk factors for ectopic pregnancy in women with symptomatic first-trimester pregnancies. Fertility and Sterility. 2006;86:36-43

[4] Bouyer J, Coste J, Fernandez H, Pouly JL, Job-Spira N. Sites of ectopic pregnancy: A 10-year populationbased study of 1800 cases. Human Reproduction. 2002;17:3224-3230

[5] Ann-Marie S, Samantha MD. Early pregnancy risks. In: Alan HD, Nathan L, editors. Current Obstetric \& Gynecologic Diagnosis \& Treatment. 11th ed. New York: McGraw-Hill; 2013. pp. 687-700

[6] Cepni I, Ocal P, Erkan S, Erzik B. Conservative treatment of cervical ectopic pregnancy with transvaginal ultrasound-guided aspiration and single-dose methotrexate. Fertility and Sterility. 2004;81:1130-1132

[7] Odejimi F, Rizzuto MI, MacRae R, Olowu O, Hussain M. Diagnosis and laparoscopic management of 12 consecutive cases of ovarian pregnancy and review of literature. Journal of Minimally Invasive Gynecology. 2009;16(3):354-359

[8] Mathur SK, Parmar P, Gupta P, Kumar M, Gilotra M, Bhatia Y. Ruptured primary ovarian ectopic pregnancy: Case report and review of the literature. Journal of Gynecologic Surgery. 2015;31(6):354-356

[9] Melcer Y, Maymon R, Vaknin Z, et al. Primary ovarian ectopic pregnancy:

Still a medical challenge. The Journal of Reproductive Medicine. 2016;61(1-2):58-62

[10] Birge O, Erkan MM, Ozbey EG, Arslan D. Medical management of an ovarian ectopic pregnancy: A case report. Journal of Medical Case Reports. 2015;9(290):1-4

[11] Goyal LD, Tondon R, Goel P, Sehgal A. Ovarian ectopic pregnancy: A 10 years' experience and review of literature. Iranian Journal of Reproductive Medicine. 2014;12(12):825-830

[12] Huber WJ, Frishman GN. Ovarian ectopic pregnancy. In: Tulandi T, editor. Ectopic Pregnancy. Cham: Springer; 2015. pp. 93-99

[13] Hosni MM, Herath RP, Mumtaz R. Diagnostic and therapeutic dilemmas of cervical ectopic pregnancy. Obstetrical \& Gynecological Survey. 2014;69(5):261-276

[14] Cunningham FG, Leveno KJ, Bloom SI, Hauth JC, Rouse DJ, Spong CY. Williams obstetrics. In: Ectopic Pregnancy. 23rd ed. New York City, USA: McGraw Hill Professional; 2010. p. 251

[15] Agonistini A, Blanc K, Ronda I. Prognostic value of human chorionic gonadotropin changes after methotrexate for ectopic pregnancy. Fertility and Sterility. 2007;88(2):504-506

[16] Ayinde OA, Aimakhu CO, Adeyanju OA, Omigbodun AO. Abdominal pregnancy at university 
college hospital, Ibadan; a ten-year review. African Journal of Reproductive Health. 2005;9(1):123-127

[17] Zhang J, Sheng Q. Full term abdominal pregnancy: A case report and review of literature. Gynecologic and Obstetric Investigation.

2008;65(2):139-141

[18] Studdiford WE. Primary peritoneal pregnancy. American Journal of Obstetrics and Gynecology. 1942;44:487-491

[19] Karat LS. Viable abdominal pregnancy. The Journal of Obstetrics and Gynecology of India. 2007;57(2):169-170

[20] Stevens CA. Malformations and deformations in abdominal pregnancy. American Journal of Medical Genetics. 1993;47(8):1189-1195

[21] Baffoe P, Fofie C, Gandau BN. Term abdominal pregnancy with healthy newborn: A case report. Ghana Medical Journal. 2011;45(2):81-83

[22] Shrestha A, Chawla CD. Ruptured primary ovarian pregnancy: A rare case report. Kathmandu University Medical Journal. 2012;10:76-77

[23] Ching H-C, Chiu T's-H, Hsieh T's-T's, Lo L-M. Early cervical ectopic pregnancy: A case report and review of literature. Journal of Medical Ultrasound. 2014;22(2):117-119

[24] Singh S. Diagnosis and management of cervical ectopic pregnancy. Journal of Human Reproductive Sciences. 2013;6(4):273-276

[25] Panelli DM, Phillips CH, Brady PC. Incidence, diagnosis and management of tubal and nontubal ectopic

pregnancies: A review. Fertility Research and Practice. 2015;1:15

[26] D’Antonio F, Palacios-Jaraquemada J, Lim PS, Forlani F, Lanzone A, Timor-Tritsch I, et al. Counseling in fetal medicine: Evidence-based to clinical answers to clinical questions on morbidly adherent placenta. Ultrasound in Obstetrics \& Gynecology. 2016;47:290-301

[27] Ash A, Smith A, Maxwell D. 2 wn location. Human Reproduction. 2006;21(10):2706-2710

[28] Arleo EK, DeFilippis EM. Cornual, interstitial, angular pregnancies: Clarifying the terms and a review of the literature. Clinical Imaging. 2014;38:763-770

[29] Condous G, Kirk E, Lu C, et al. There is no role for uterine curettage in the contemporary diagnostic workup of women with a pregnancy of unknown location. Human Reproduction. 2006;21(10):2706-2710

[30] Hahlin M, Thorburn J, Bryman I. The expectant management of early pregnancies of uncertain site. Human Reproduction. 1995;10:1223-1227

[31] Hammoud AO, Hammoud I, Bujold E, et al. The role of sonographic endometrial patterns and endometrial thickness in the differential diagnosis of ectopic pregnancy. American Journal of Obstetrics and Gynecology. 2005;192:1370-1375

[32] Rosen T. Placenta accreta and cesarean scar pregnancy: Overlooked costs of the rising cesarean section rate. Clinics in Perinatology. 2008;35:519-529

[33] Maymon R, Halperin R, Mendlovic S, Schneider D, Herman A. Ectopic pregnancies in a caesarean scar: Review of the medical approach to an iatrogenic complication. Human Reproduction Update. 2004;10:515-523

[34] Stovall TG, Ling FW, Gray LA. Single-dose methotrexate treatment of ectopic pregnancy. Obstetrics and Gynecology. 1991;77:754-757 
[35] Lipscomb GH, McCord ML, Stovall TG, Huff G, Portera SG, Ling FW. Predictors of success of methotrexate treatment in women with tubal ectopic pregnancies. The New England Journal of Medicine. 1999;341:1974-1978

[36] Protocol of Gynecological Service, University of Alabama at Birmingham, December 2011

[37] Barnhart KT, Franasiak JM.

American College of Obstetrician and Gynecologist Practice Bulletin Number 193. Tubal ectopic pregnancy. Obstetrics and Gynecology. 2018;121(3):e91-e103

[38] Monteagudo A, Minior VK, Stephenson C, Monda S, Timor-Tritsch IE. Non-surgical management of live ectopic pregnancy with ultrasoundguided local injection: A case series. Ultrasound in Obstetrics \& Gynecology. 2005;25(3):282-288

[39] Verma U, Jacques E. Conservative management of live tubal pregnancies by ultrasound-guided potassium chloride injection and systematic methotrexate treatment. Journal of Clinical Ultrasound. 2005;33(9):460-463

[40] Rozenberg P, Chevrets S, Camus E, De Tayrac R, Garbin O, de Poncheville L, et al. Medical treatment of ectopic pregnancies: A randomized clinical trial comparing methotrexatemifepristone and methotrexate Placebo. Human Reproduction. 2003;18:1802-1808

[41] Perdu M, Camus E, Rozenberg P, Goffinet F, Chastang C, Philippe HJ, et al. Treating ectopic pregnancy with combination of mifepristone and methotrexate: A phase II nonrandomised study. American Journal of Obstetrics and Gynecology. 1998;179(3 pt 1):640-643

[42] Nilsson UW, Johns TG, Wilmann T, Kaitu'u-Lino T, Whitehead C, Dimitriadis E, et al. Effects of gefitinib, an epidermal growth factor receptor inhibitor, on human placental cell growth. Obstetrics and Gynecology. 2013;122(4):737-744

[43] Skubisz MM, Horne AW, Johns TG, Nilsson UW, Duncan WC, Wallace EM, et al. Combination gefitnib and methotrexate compared with methotrexate alone to treat ectopic pregnancy. Obstetrics and Gynecology. 2013;122(4):745-751

[44] Jurkovic D, Hillaby K, Woelfer B, Lawrence A, Salim R, Elson CJ. Firsttrimester diagnosis and management of pregnancies implanted into the lower uterine segment cesarean section scar. Ultrasound in Obstetrics \& Gynecology. 2003;21:220-227

[45] Harb HM, Knight M, Bottomley C, Overton C, Tobias A, Gallos ID, et al. Caesarean scar pregnancy in the UK: A national cohort study. BJOG. 2018;125:1663-1670

[46] Jurkovic D, Knez J, Appiah A, Farahani L, Mavrelos D, Ross JA. Surgical treatment of cesarean scar ectopic pregnancy: Efficacy and safety of ultrasound-guided suction curettage. Ultrasound in Obstetrics \& Gynecology. 2016;47(4):511-517

[47] Vial Y, Petignat P, Hohlfeld P.

Pregnancy in a cesarean scar. Ultrasound in Obstetrics \& Gynecology. 2000;16(6):595-593

[48] Liao CY, Tse J, Sung SY, Chen SH, Tsui WH. Cornual wedge resection for interstitial pregnancy and postoperative outcome. Australian and New Zealand Journal of Obstetrics and Gynaecology. 2017;57(3):342-345

[49] Kraemer B, Kraemer E, Guengoer E, Juhasz-Boess I, Solomayer EF, Wallwiener D, et al. Ovarian ectopic pregnancy; diagnosis, treatment to Carnegie stage 16 and review based on clinical case. Fertility and Sterility. 2009;9:393.e13-393.e15 


\title{
Cervical Pregnancy Diagnosis and Management
}

\author{
Rubens Bermudes Musiello, Jair Luiz Fava, Luiz Camano \\ and Eduardo de Souza
}

\begin{abstract}
Cervical ectopic pregnancy is rare. Cervical implantation is a serious obstetric condition that can lead to mutilation if not promptly diagnosed and treated. The clinical diagnosis consists in physical examination, ballooned cervical canal, dilated cervix, and hourglass-shaped uterus detected by internal examination. Ultrasonography confirms the diagnosis by visually detecting cervical implantation below the internal orifice. Magnetic resonance imaging and three-dimensional ultrasonography validates the diagnosis. Conservative treatment proposed: cerclage, vasopressin, Foley catheter with hemostatic cervical ligation, cervical canal balloon, embolization of uterine artery, hysteroscopy, laparoscopic ligation of the uterine artery, and curettage by suction catheter. Shortcomings of conservative management are high, and a number of cases result in hysterectomy. Drug management is a therapeutic option to avoid mutilating surgery, and the most commonly used is systemic methotrexate (MTX). Local treatment with MTX and potassium chloride injection guided by transvaginal ultrasound is used when the embryo is alive.
\end{abstract}

Keywords: pregnancy, ectopic, cervical, methotrexate

\section{Introduction}

Ectopic pregnancy (EP) is a pregnancy in which the fertilized egg attaches itself and develops outside the uterus.

Cervical ectopic pregnancy (CEP) is considered to be exceptionally rare, and it accounts for less than $1 \%$ of ectopic pregnancies (EP) [1, 2]. Its exact incidence is not yet known; some authors report from 1:978 to 1:50,000 [1,3-8], and other authors report $0.1 \%$ of all EP, estimating their incidence between 1:2500 and 1:98,000 pregnancies [9].

The first description of cervical pregnancy dates back to 1817 in England by Sir Everard Home [10], though the term was first used by Rokitansky in 1860 [11] (Figure 1).

The implantation represents a serious obstetric event $[12,13]$, to an extent that in 1953, Baptiste reflected: "Most great obstetricians have never seen a case of CEP; however, a minority who had this opportunity wish they had never seen it."

Some risk factors have been considered for cervical nidation of the egg: anatomical abnormalities, uterine leiomyomas, synechia, previous cervical interventions leading to cervical mucosal changes, cervical stenosis, in vitro fertilization with embryo transfer, and obstetric uterine curettage [14]. 

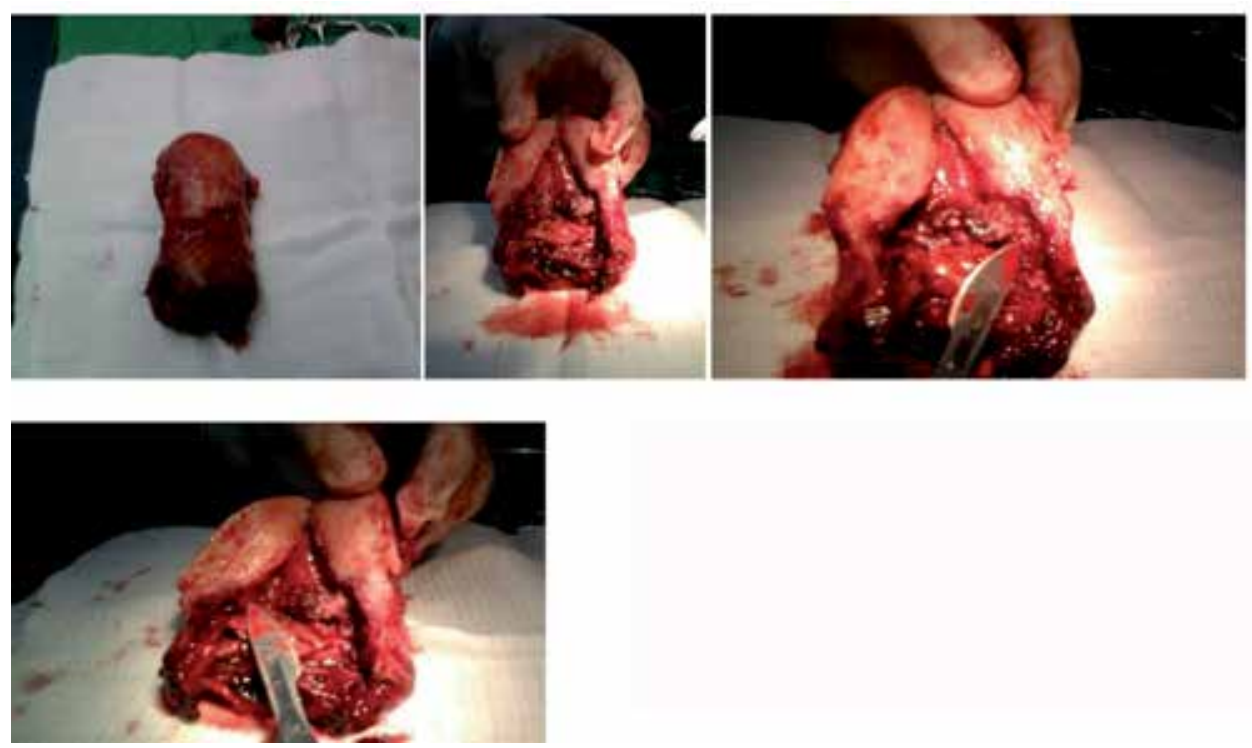

Figure 1.

A case where hysterectomy was inevitable due to the hemorrhagic condition-The trophoblastic invasion in the endocervix can be seen (own source).

When the zygote attaches itself in the cervical canal, it is rapidly invaded by the trophoblast, reaching the conjunctival portion of the cervix, once there is no adequate decidualization. As the pregnancy develops in the cervix, bleeding could occur, initially in small amounts and later in greater volume when it reaches larger vessels. These pregnancies last, in average, 7-10 weeks [14].

\section{Clinical diagnosis}

The clinical diagnosis consists in the physical examination where a ballooned cervical canal and a dilated cervix can be detected [15] and hourglass-shaped uterus can be observed by internal examination [8] (Figure 2).

The differential clinical diagnosis between CEP and the ongoing miscarriage is that in the former, the body of the uterus tends to be larger, with dilation of the

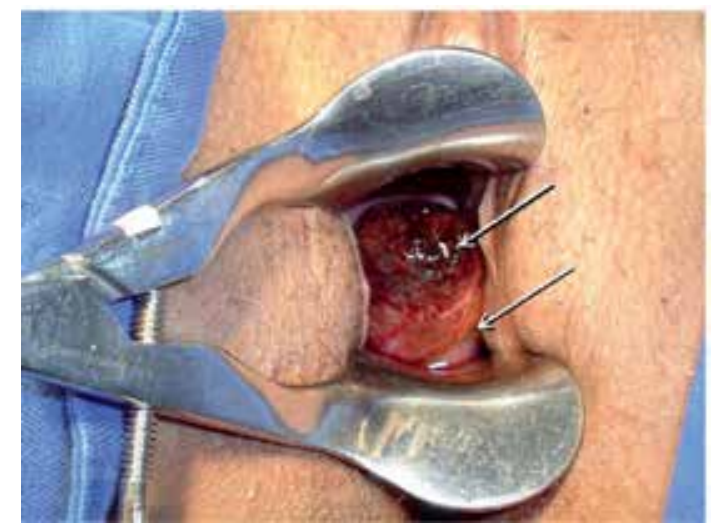

Figure 2.

Clinical diagnosis: Physical examination-Arrow at the bottom shows external orifice of the cervix, and the arrow at the top shows development of cervical pregnancy in the anterior lip of the cervix. 
external and internal orifice of the cervix, and it is possible to detect placental tissue above the internal orifice; in the latter, the internal orifice is virtually closed [16].

\section{Imaging}

More recently, ultrasonography has been confirming the diagnosis by visually detecting cervical implantation below the internal orifice (IO) $[17,18]$. Transvaginal ultrasonography is the gold standard preliminary method for accurate diagnosis [19].

The ultrasound findings for the diagnosis of cervical pregnancy are empty uterine cavity, endometrial thickness due to decidual reaction, hourglass shape of the uterus, enlargement of cervical canal, gestational sac located in the cervix with or without embryo, placental tissue surrounding the gestational sac, and closed internal orifice of the cervix (Figure 3).

Three-dimensional ultrasonography is also useful in the diagnosis of GC [20, 21]. Magnetic resonance imaging validates the diagnosis [22, 23] (Figure 4).
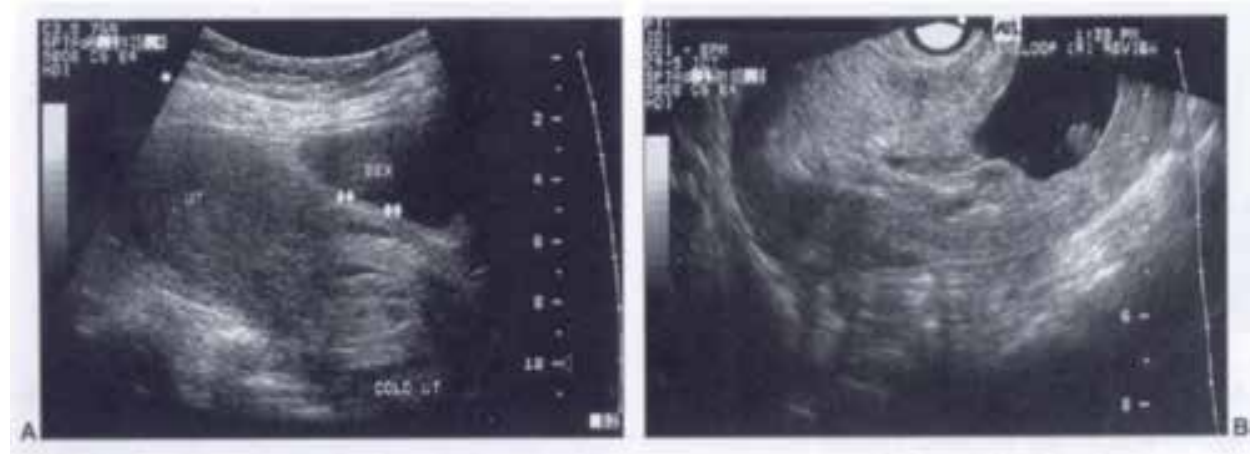

Figure 3.

Diagnosis by imaging - Ultrasonography showing ovular implantation below internal orifice of the cervix.

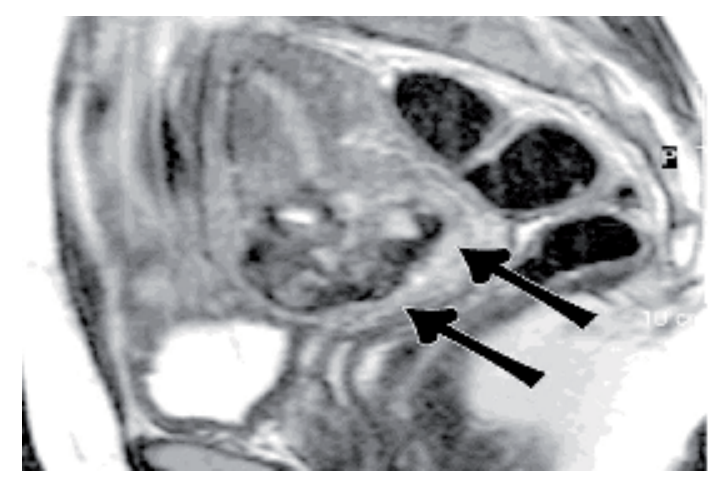

Figure 4.

Diagnosis by imaging - MRI. Arrows show pregnancy developing in the cervical region, below the IO. Source: Bader-Armstrong et al. [22].

\section{Management}

The classical treatment used to be total hysterectomy due to profuse bleeding. However, some authors, eager to keep their nulliparous patients' fertility, tried the conservative treatment of uterine curettage or local suture $[15,16,24]$. 
Reviewing the literature CEP was only cited only in the ninth edition of Williams Obstetrics in 1945. The treatment proposed at that time was [25] blood transfusion, vaginal tamponade and hysterectomy [26].

\section{Conservative surgical treatment}

The conservative surgical treatment proposed in the literature are:

1. Cerclage [27]

2. Foley catheter with hemostatic cervical ligation [28, 29]

3. Uterine curettage by suction catheter [30-33]

4. Hysteroscopy $[19,34]$

5. Laparoscopic ligation of the uterine artery [35]

However, the majority of the conservative surgical treatments fail and result in hysterectomy.

\section{Medical treatment}

The following drugs are used in the medical treatment:

1. Vasopressin [36]

2. Actinomycin D and cyclophosphamide [37]

3. etoposide [38]

4. Intra-amniotic and systemic methotrexate (MTX) and potassium chloride with live embryo $[39,40]$

5. Intramuscular methotrexate with dead embryo [41]

Among the drugs mentioned above, methotrexate (MTX) is the most used in the current literature for the treatment of EP. It is an antimetabolic chemotherapeutic and folic-acid antagonist which operates through the competitive inhibition of the dihydrofolate reductase enzyme, which in turn reduces the dihydrofolic acid into folinic acid [42], interfering the DNA synthesis and, consequently, the cell division.

Our experience advocates the therapy using a single dose of MTX as first-line treatment for CEP if the embryo does not show cardiac activity.

In cases of embryos without heart beating with high $\beta$-hcg titers greater than $5000 \mathrm{mui} / \mathrm{ml}$ and often greater than $10,000 \mathrm{mui} / \mathrm{ml}$, a systemic treatment with multiple doses of MTX is the preferred therapeutic.

When the embryo has cardiac activity, local treatment with MTX and potassium chloride is recommended [40]. Elito et al. published a series of eight cases of CEP where embryos are presenting cardiac activity treated with puncture of gestational sac guided by vaginal ultrasonography with MTX injection $(1 \mathrm{mg} / \mathrm{kg})$ and $\mathrm{KCl}$ injection [42]. 


\section{Expectant management}

We can rarely adopt the expectant management, monitoring throughout the development of the cervical pregnancy, as some cases can spontaneously solve itself. Patients with small-volume CEP and low and declining serum beta-hCG concentrations are candidates for this type of management. Titers of beta-hCG lower than $1500 \mathrm{mIU} / \mathrm{ml}$ and declining indicate a high possibility of spontaneous involution.

\section{Conclusion}

According to the historical evolution of CEP, the treatment was carried out with total abdominal hysterectomy in a heroic attempt to solve the hemorrhage after the rupture of the cervix. When the authors began to try a traditional treatment, it was usually a surgery. Early diagnosis of CEP is extremely important for choosing the appropriate treatment. Several treatment options can be used, such as surgical interventions, medical treatment, and expectant management. The treatment with MTX has a high rate of success, and it is the first choice. CEP with cardiac activity is treated with intra-aminiotic MTX and potassium chloride injection. In cases of CEP without heart beating, systemic treatment with MTX should be used in a single- or multiple-dose regimen depending on the levels of beta-hCG. When the levels of beta-hCG are low and declining, the expectant management may be chosen. The clinical treatment of CEP prevents mutilating surgeries, and the patient can preserve their original condition for an obstetric future.

\section{Author details}

Rubens Bermudes Musiello, ${ }^{1,2}$, Jair Luiz Fava ${ }^{1,2}$, Luiz Camano ${ }^{2}$ and Eduardo de Souza ${ }^{2}$

1 Escola Superior de Ciências da Santa Casa de Misericórdia de Vitória, EMESCAM, Brasil

2 Escola Paulista de Medicina - Universidade Federal de São Paulo - UNIFESP, Brasil

*Address all correspondence to: rubens.musiello@gmail.com

IntechOpen

(C) 2019 The Author(s). Licensee IntechOpen. This chapter is distributed under the terms of the Creative Commons Attribution License (http://creativecommons.org/licenses/ by/3.0), which permits unrestricted use, distribution, and reproduction in any medium, provided the original work is properly cited. (cc) BY 


\section{References}

[1] Parente JT, Ou CS, Levy J, Legatt E. Cervical pregnancy analysis: A review and report of five cases. Obstetrics and Gynecology. 1983;62:79-82

[2] Pisarska M, Carson S. Incidence and risk factors for ectopic pregnancy. Clinical Obstetrics and Gynecology. 1999;42(1):2-8; quiz 55-56

[3] Dees HC. Cervical pregnancy associated with uterine leiomyomas. Southern Medical Journal. 1966;59(8):900; passim

[4] Shinagawa S, Nagayama M. Cervical pregnancy as a possible sequela of induced abortion. Report of 19 cases. American Journal of Obstetrics and Gynecology. 1969;105(2):282-284

[5] Ushakov FB, Elchalal U, Aceman PJ, Schenker JG. Cervical pregnancy: Past and future. Obstetrical \& Gynecological Survey. 1997;52(1):45-59

[6] Bai SW, Lee JS, Park JH, Kim JY, Jung KA, Kim SK, et al. Failed methotrexate treatment of cervical pregnancy. Predictive factors. The Journal of Reproductive Medicine. 2002;47(6):483-488

[7] Jozwiak EA, Ulug U, Akman MA, Bahceci M. Successful resection of a heterotopic cervical pregnancy resulting from intracytoplasmic sperm injection. Fertility and Sterility. 2003;79(2):428-430

[8] Papaloucas CD. "Hour-glass” shape of the uterus in the diagnosis and treatment of cervical pregnancy. Clinical Anatomy. 2004;17(8):658-661

[9] Alanis-Fuentes J, Brindis-Rodríguez A, Martínez-Arellano M. Cervical ectopic pregnancy. Hysteroscopy treatment, case report. Ginecología y Obstetricia de México. 2015;83(5):302-307
[10] Dreizin DH, Schneider P. Cervical pregnancy. The American Surgeon. 1957;93(1):27-40

[11] Ranade V, Palermino A, Tronik B. Cervical pregnancy. Obstetrics and Gynecology. 1978;51(4):502-503

[12] Rubin IC. Cervical pregnancy. Surgery, Gynecology \& Obstetrics. 1911;13(s/n):625-633

[13] Studdiford WE. Cervical pregnancy: A partial review of the literature and a report of two probable cases. American Journal of Obstetrics and Gynecology. 1945;49(2):169-185

[14] Musiello RB, Junior JE, Musiello RB, Camano L, Souza E, Fava JL, et al. Cervical ectopic pregnancy with live embryo: The use of methotrexate is effective? 2012:2-4

[15] Camano L, Azevedo AR. Prenhez ectópica. In: Delascio D, editor. Síndromes Hemorrágicas da Gestação. São Paulo: Sarvier; 1977. pp. 63-78

[16] Greenhill JP, Friedman EA. Prenhez ectopica. In: Obstetricia. Rio de Janeiro: Interamericano; 1976. pp. 351-395

[17] Frates MC, Benson CB, Doubilet PM, Di Salvo DN, Brown DL, Laing FC, et al. Cervical ectopic pregnancy: Results of conservative treatment. Radiology. 1994;191(3):773-775

[18] Kligman I, Adachi TJ, Katz E, McClamrock HD, Jockle GA, Barakat B. Conserving fertility with early management of cervical pregnancy: A case report. The Journal of Reproductive Medicine. 1995;40(10):743-746

[19] Mangino FP, Ceccarello M, Di Lorenzo G, D’Ottavio G, Bogatti P, 
Ricci G. Successful rescue hysteroscopic resection of a cervical ectopic pregnancy previously treated with methotrexate with no combined safety precautions. Clinical and Experimental Obstetrics \& Gynecology. 2014;41(2):214-216

[20] Ruano R, Reya F, Picone O, Chopin N, Pereira PP, Benach A, et al. Three-dimensional ultrasonographic diagnosis of a cervical pregnancy. Clinics (São Paulo). 2006;61(4): 355-358

[21] Sherer DM, Gorelick C, Dalloul M, Sokolovski M, Kheyman M, Kakamanu S, et al. Three-dimensional sonographic findings of a cervical pregnancy. Journal of Ultrasound in Medicine. 2008;27(1):155-158

[22] Bader-Armstrong B, Shah Y, Rubens D. Use of ultrasound and magnetic resonance imaging in the diagnoses of cervical pregnancy. Journal of Clinical Ultrasound. 1989;17(4):283-286

[23] Rafal RB, Kosovsky PA, Markisz JA. Case report. MR appearance of cervical pregnancy. Journal of Computer Assisted Tomography. 1990;14(3):482-484

[24] Neme B, Pereira PP, Zugaib M. Prenhez ectópica. In: Neme B, editor. Obstetricia Básica. 3rd ed. São Paulo: Sarvier; 2006. pp. 372-394

[25] Studdiford WE. Cervical pregnancy. American Journal of Obstetrics and Gynecology. 1945;49:169

[26] Stander HJ. Extra uterine pregnancy. In: Willians Obstetrics. 9th ed. New York: D. Appleton - Century Company; 1945. pp. 752-783

[27] Bernstein D, Holzinger M, Ovadia J, Frishman B. Conservative treatment of cervical pregnancy. Obstetrics and Gynecology. 1981;58(6):741-742
[28] Nolan TE, Chandler PE, Hess LW, Morrison JC. Cervical pregnancy managed without hysterectomy. A case report. The Journal of Reproductive Medicine. 1989;34(3):241-243

[29] Thomas RL, Gingold BR, Gallagher MW. Cervical pregnancy. A report of two cases. The Journal of Reproductive Medicine. 1991;36(6):459-462

[30] Lobel SM, Meyerovitz MF, Benson CC, Groff B, Bengtson JM. Preoperative angiographic uterine artery embolization in the management of cervical pregnancy. Obstetrics and Gynecology. 1990;72:938-941

[31] Simon P, Donner C, Delcour C, Kirkpatrick C, Rodesch F. Selective uterine artery embolization in the treatment of cervical pregnancy: Two case reports. European Journal of Obstetrics, Gynecology, and Reproductive Biology. 1991;40(2):159-161

[32] Fylstra DL. Cervical pregnancy: 13 cases treated with suction curettage and balloon tamponade. American Journal of Obstetrics and Gynecology. 2014;210(6):581.e1-581.e5

[33] Takeda K, Mackay J, Watts S. Successful management of cervical ectopic pregnancy with bilateral uterine artery embolization and methotrexate. Case Reports in Emergency Medicine. 2018;3:9593824

[34] Tanos V, ElAkhras S, Kaya B. Hysteroscopic management of cervical pregnancy: Case series and review of the literature. Journal of Gynecology Obstetrics and Human Reproduction. 2018;48(4):247-253

[35] Kung FT, Lin H, Hsu TY, Chang CY, Huang HVV, Huang LY, et al. Differential diagnosis of suspected cervical pregnancy and conservative 
treatment with the combination of laparoscopy-assisted uterine artery ligation and hysteroscopic endocervical resection. Fertility and Sterility.

2004;81(6):1642-1649

[36] Wharton KR, Gore B. Cervical pregnancy managed by placement of a shirodkar cerclage before evacuation. A case report. The Journal of Reproductive Medicine. 1988;33(2):227-229

[37] Bakri YN, Badawi A. Cervical pregnancy successfully treated with chemotherapy. Acta Obstetricia et Gynecologica Scandinavica. 1990;69(7):655-656

[38] Segna RA, Mitchell DR, Misas JE. Successful treatment of cervical pregnancy with oral etoposide. Obstetrics and Gynecology. 1990;76(5):945-947

[39] Arowojolu AO, Ogunbode OO. Cervical ectopic pregnancy managed with methotrexate and tranexamic acid: A case report. African Journal of Medicine and Medical Sciences. 2014;43(4):361-364

[40] Junior JE, Musiello RB, Araújo Junior E, Souza E, Fava JL, Guerzet EA, et al. Conservative management of cervical pregnancy heart activity by ultrasound-guided local injection: An eighth case series. The Journal of Maternal-Fetal \& Neonatal Medicine. 2014;27(13):1378-1381

[41] Zugaib M. Zugaib Obstetrícia. 3rd ed. São Paulo: Manole; 2016. 1348p

[42] Cecchino N, Araújo Junior E, Elito Junior J. Methotrexate for ectopic pregnancy: When and how. Archives of Gynecology and Obstetrics.

2014;2903(3):417-423 


\title{
Caesarean Scar Ectopic Pregnancy
}

\author{
Workineh Getaneh Tadesse
}

\begin{abstract}
Caesarean scar ectopic pregnancy (CSEP) is one of the rarest forms of ectopic pregnancy. It is characterised by a gestation implanted in a caesarean section scar and surrounded by the myometrium and the fibrous tissue of the scar. With rising caesarean delivery rates and widespread use of ultrasound in early pregnancy, there is a parallel upward trend in the incidence of CSEP. The most significant risk associated with a CSEP is an early uterine rupture with life-threatening maternal haemorrhage leading to hysterectomy and loss of reproductive potential. An early diagnosis can offer conservative treatment options capable of preserving the uterus. This chapter discusses the clinical presentation, diagnosis and evidence-based treatment options of CSEP.
\end{abstract}

Keywords: scar ectopic pregnancy, caesarean section, complications of early pregnancy, uterine rupture

\section{Introduction}

CSEP is one of the rarest forms of ectopic pregnancies [1-5]. However, in the last two to three decades, an increasing number of case reports and case series have been published [4]. CSEP results from the implantation of the embryo in a previous caesarean scar (CS) instead of the endometrial cavity [6]. The pregnancy is surrounded by the myometrium and the fibrous tissue of the scar. It usually manifests with painless vaginal bleeding and often misdiagnosed as spontaneous miscarriage or cervical ectopic pregnancy $[1,4]$. A misdiagnosis or a late diagnosis of CSEP can result in early uterine rupture with life-threatening maternal haemorrhage leading to hysterectomy and loss of reproductive potential [6]. Ultrasonography with colour flow Doppler is the primary diagnostic tool in the workup of CSEP [4]. An early diagnosis can offer conservative treatment options that enable the preservation of the uterus $[2,5,6]$.

\section{Epidemiology}

The exact incidence of CSEP is unknown. It is estimated to occur in $0.05-0.4 \%$ ( 1 in 1800 to 1 in 2500) of all pregnancies [1]. A recent publication from the UK's Early Pregnancy Surveillance Service (UKEPSS) reported an estimated incidence of 1.5 per 10,000 maternities [7]. The incidence of CSEP is rising, which is believed to be due to the increasing caesarean delivery rate itself and improved detection rate as a result of widespread use of ultrasonography during early pregnancy [8] .

CSEP has been reported in women with only one previous caesarean delivery as well as in those with multiple caesarean deliveries. It is not clear whether the 
number of previous caesarean delivery (CD) or the time interval between the CD and subsequent pregnancy affect the incidence of CSEP. Similar to other types of ectopic pregnancies, CSEP has been reported in both spontaneously conceived pregnancy as well as after in vitro fertilisation (IVF) and embryo transfer $[9,10]$.

\section{Pathophysiology}

The exact cause and mechanism are not well understood. The most plausible mechanism is that the blastocyst implants into microtubular tracts, which persisted at the site of the caesarean scar as a result of incomplete healing [1]. However, it is not clear if an increase in the number of previous caesarean deliveries or short interval between caesarean delivery and subsequent pregnancy increase the risk of CSEP by way of affecting the healing process at the caesarean scar. Additionally, there is no evidence if uterine closure technique (e.g. single vs. double layer closure) has any effect on the risk of CSEP.

Two types of CSEP have been described [8]. Type I (endogenic type) is characterised by implantation on the CS with progression towards the direction of the endometrial cavity. Type II (exogenic type) CSEP is a deep implantation in the CS defect growing mainly towards the abdominal cavity. Type I CSEP can potentially continue to develop into the endometrial cavity and may even progress to the second and third trimesters. Type II is associated with early uterine rupture or invasion into the urinary bladder [8].

CSEP should be differentiated from placenta accreta or increta, where the pregnancy is intrauterine with an invasion of the trophoblast into the myometrium. In contrast, CSEP is characterised by a gestation separated from the endometrial cavity and surrounded by the myometrium and the fibrous tissue of the scar.

\section{Clinical presentation and diagnosis}

A large proportion of patients with CSEP are asymptomatic and diagnosed incidentally during a routine first-trimester ultrasound $[1,4]$. The most frequent symptom of CSEP is light and painless vaginal bleeding [4, 6]. Some patients may have associated mild to moderate lower abdominal pain. Severe pain with or without haemodynamic instability, may indicate ruptured CSEP. There may be mild lower abdominal or uterine tenderness on palpation; however, clinical examination in stable women is usually unremarkable. The gestational age at diagnosis ranges from 5 to 16 weeks, with an average of $7.5 \pm 2.5$ weeks [4].

\subsection{Investigations}

\subsubsection{Serum beta - human chorionic gonadotropin ( $h C G$ )}

Quantitative serum beta-hCG measurement confirms the presence of pregnancy, and also provides a baseline for posttreatment follow up.

\subsubsection{Ultrasound}

Ultrasonography is the primary diagnostic tool $[1,4,6]$. Transvaginal ultrasound (TVS) has a diagnostic sensitivity of around $85 \%[1,4]$. There are 
proposed ultrasound diagnostic criteria, allowing a differential diagnosis from cervical ectopic pregnancy and miscarriage. These include (1) Empty uterus with clearly visualised endometrium, (2) Empty cervical canal, (3) Gestational sac located within the anterior isthmic portion of the uterus at the presumed site of the CS, (4) Thinned ( $<5 \mathrm{~mm}$ ) or absent myometrium between the gestational sac and bladder [5]. Additionally, Doppler colour flow may show distinct circular peritrophoblastic perfusion surrounding the gestational sac (see Figures 1 and 2).

The crossover sign (COS) is an ultrasound sign that is proposed to describe the relationship between the gestational sac, the caesarean scar and the anterior uterine wall [12]. In a sagittal view of the uterus a straight line connecting the internal os and the uterine fundus through the endometrium is drawn (endometrial line). The superior-inferior (S-I) diameter of the gestational sac, perpendicular to the endometrial line, is traced (see Figure 3).

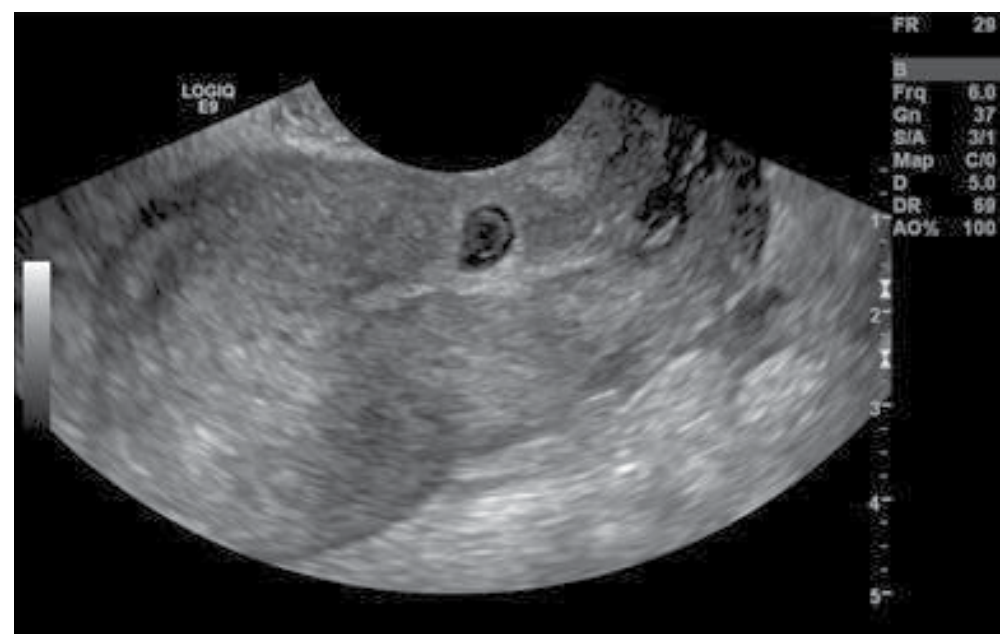

Figure 1.

Transvaginal ultrasound, sagittal view of a gestational sac with a yolk sac within a caesarean scar [11].

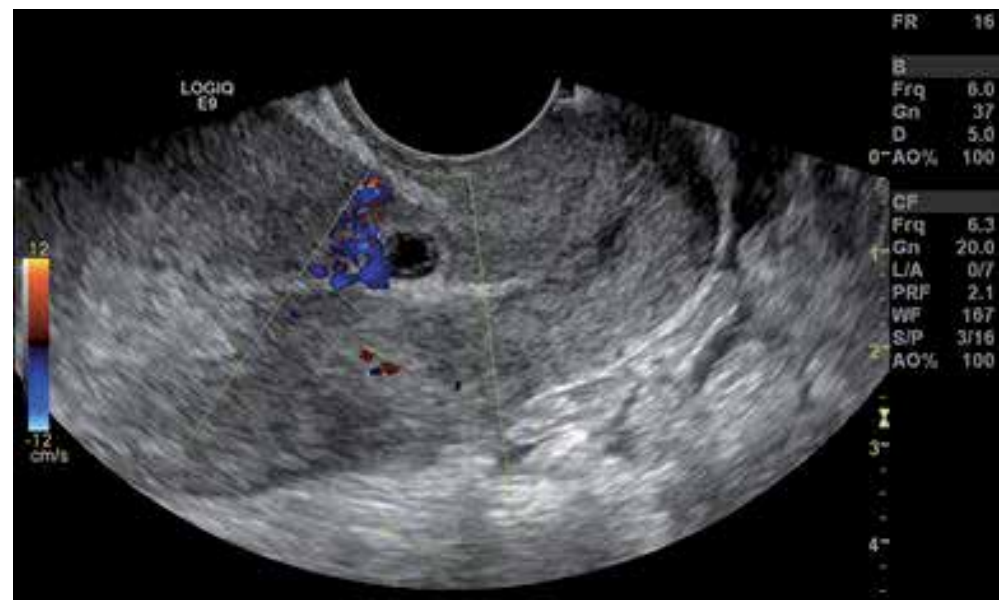

Figure 2.

Transvaginal ultrasound, sagittal view with colour Doppler demonstrating peritrophoblastic perfusion [11]. 
Patients can be categorised into groups based on the relationship between the endometrial line and the S-I diameter of the gestational sac [12]:

1. Normal - gestational sac implanted away from the caesarean scar and close to the fundus.

2. COS-1 - gestational sac implanted within the caesarean scar and at least twothird of the S-I diameter of the gestational sac is above the endometrial line.

3. COS-2+ - gestational sac implanted within the caesarean scar and less than two-third of the S-I diameter of the gestational sac is above the endometrial line.

4. COS-2- - gestational sac implanted within the caesarean scar and less than two-third of the S-I diameter of the gestational sac is above the endometrial line, but there is no intersection between the S-I diameter and the endometrial line (see Figure 4).

This categorisation based on the COS may help to determine the natural progression of CSEP [12].

\subsubsection{Magnetic resonance imaging}

Magnetic resonance imaging (MRI) is rarely required to clarify the diagnosis of CSEP [1]. Sagittal, coronal and transverse sections of T1- and T2- weighted MRI sequences can clearly show the gestational sac embedded in the anterior lower uterine wall [5] (Figure 5). MRI can also help in decision making and pre-operative planning through detailed characterisation of CSEP location, depth of myometrial invasion and presence of bladder involvement $[1,5]$ (Figure 6).

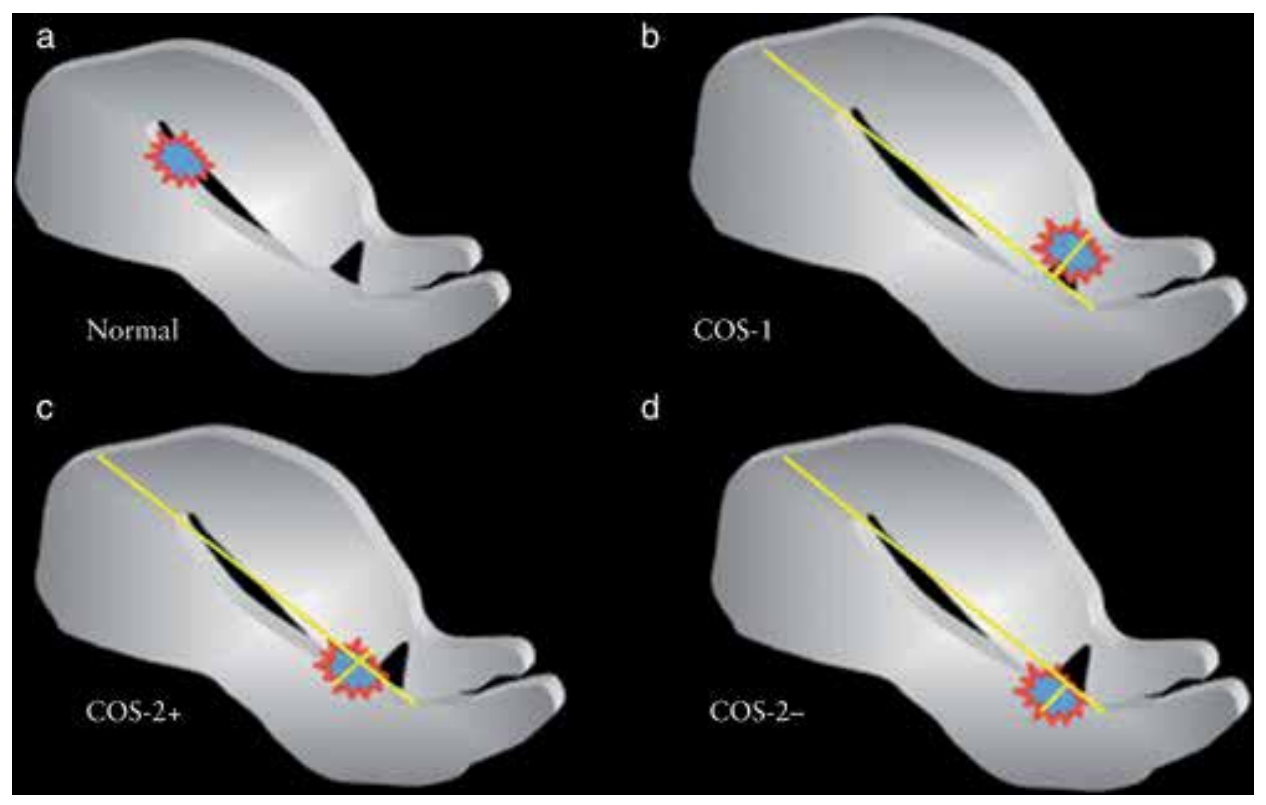

Figure 3.

Diagrammatic representation of relationship between gestational sac, caesarean scar and anterior uterine wall, defined as crossover sign (COS) [12]. (a) Normal implantation, (b) COS-1, (c) COS-2+, (d) COS-2-. 
Caesarean Scar Ectopic Pregnancy

DOI: $h t t p: / / d x$. doi.org/10.5772/intechopen.89023
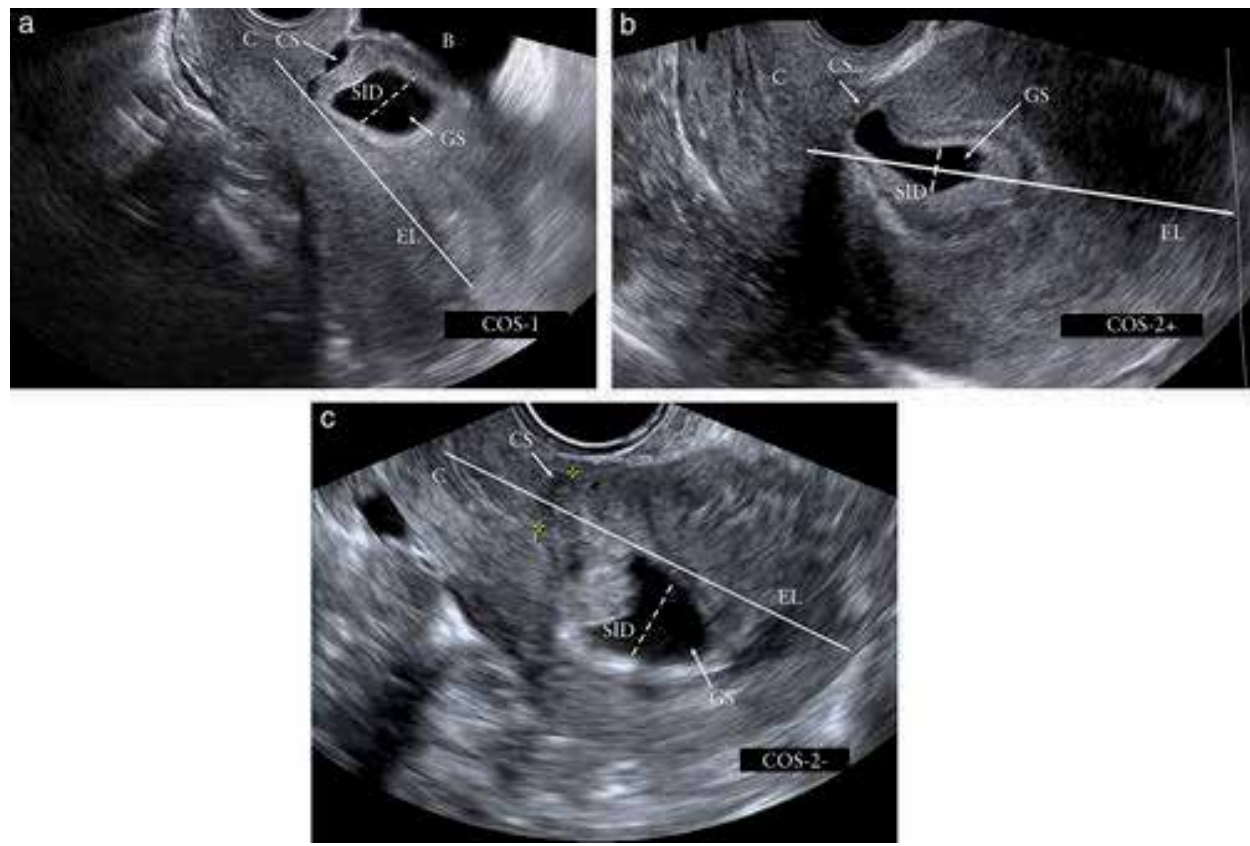

Figure 4.

Ultrasound images of different types of crossover sign (COS): (a) COS-1, (b) COS $-2^{+}$and (c) $\mathrm{COS}_{-2}{ }^{-}$. $B=$ bladder, $C=$ cervix, $C S=$ caesarean scar, $E L=$ endometrial line, $G S=$ gestational sac, $S I D=$ superiorinferior diameter [12].

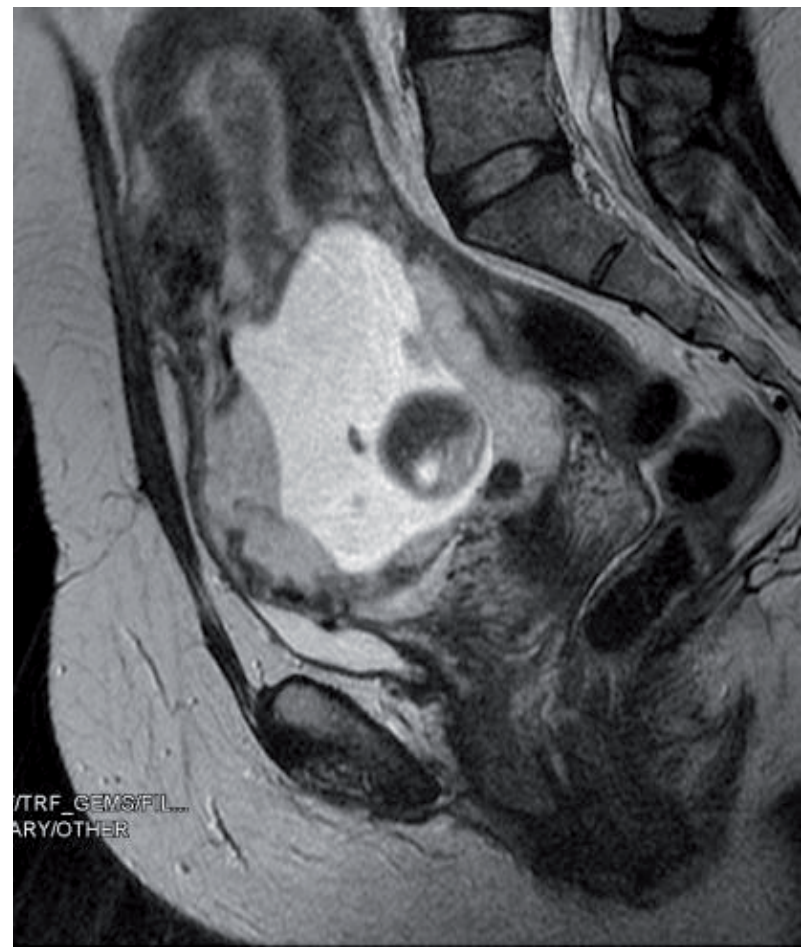

Figure 5.

MRI from a case of a 2nd trimester CSEP (see Figure 6 for the surgical specimen from the same patient). 


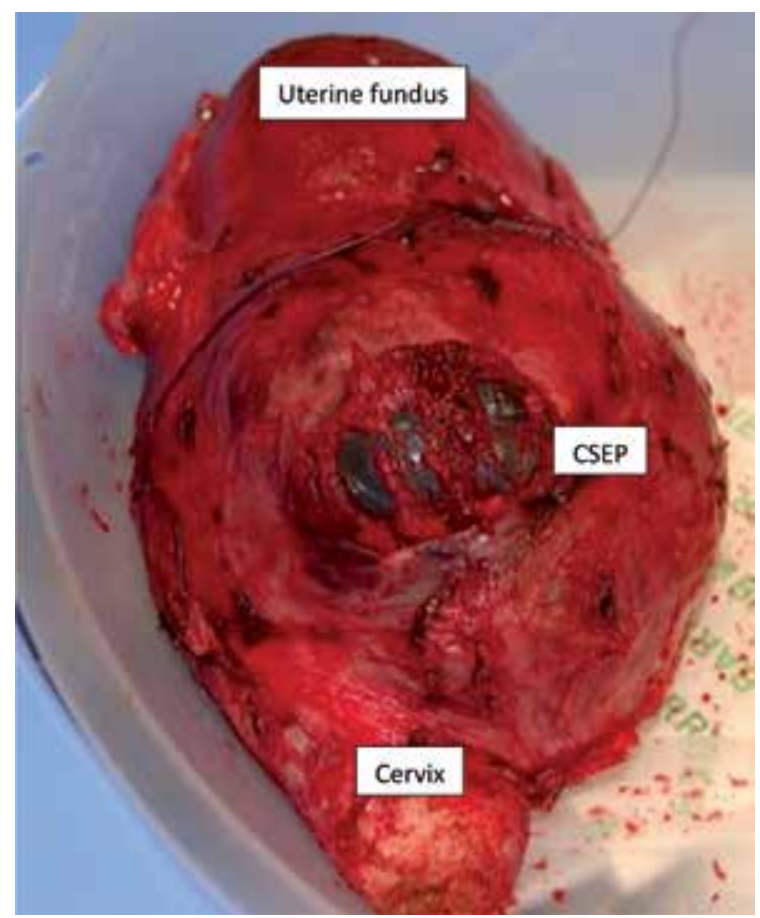

Figure 6.

Surgical specimen of a total hysterectomy from a case of a 2nd trimester CSEP. Note the distended lower segment of the uterus with near total absence of myometrium at the previous caesarean scar.

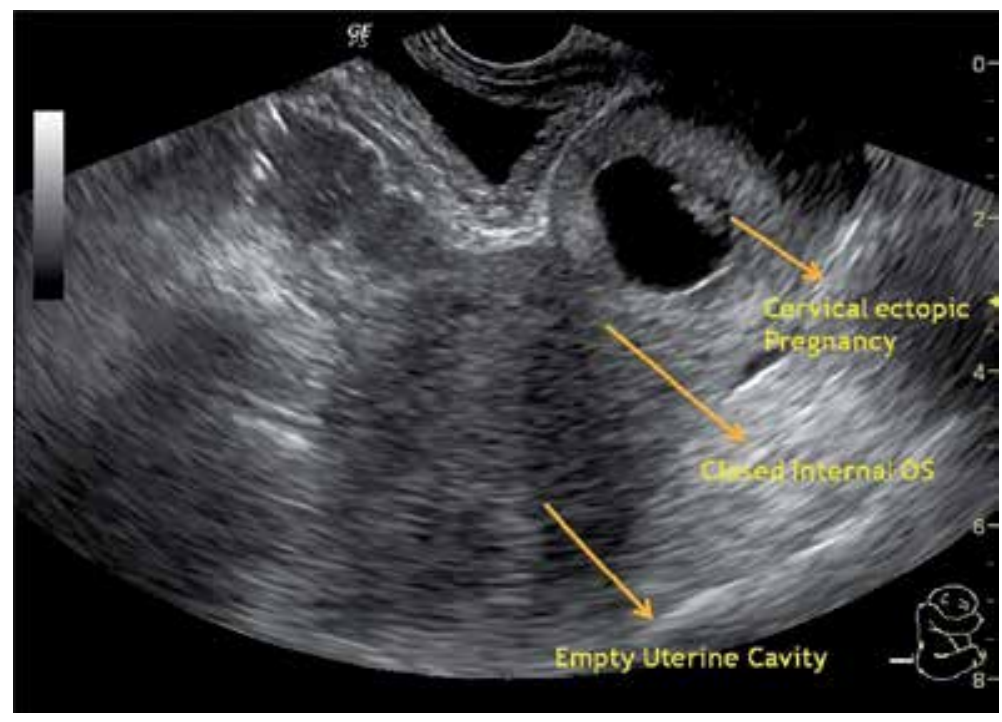

\section{Figure 7.}

Cervical ectopic pregnancy: Hourglass appearance of the uterus with gestational sac in the intracervical canal [14].

\subsection{Differential diagnoses}

CSEP can be mistaken for spontaneous miscarriage in progress $[11,13]$ or cervical ectopic pregnancy [13]. In cervical pregnancy, unlike CSEP, there would be a layer of myometrium visible between the bladder and the gestational sac and bleeding as the presenting symptom is much more substantial $[5,6,11,13]$. The uterus 
is empty with an endometrial stripe, the gestational sac presents predominantly within the cervix, giving an hour-glass shape of the uterus with a ballooned cervical canal (see Figure 7) [5, 6, 14]. In case of spontaneous miscarriage in progress, an avascular gestational sac is seen in the cervical canal indicating that it has been detached from its implantation site, in contrast to the well-perfused CSEP located in the anterior lower uterine wall $[5,13]$. A significant fall in serum beta-hCG level would also be noticed in a miscarriage.

\section{Treatment}

Due to the rarity of CSEP, there is no consensus regarding its management. Treatment options discussed here are mostly based on case reports and small case series. In general, there are expectant, medical and surgical options of treatment. Factors influencing the choice of treatment options include the type of the CSEP (Type I or II), haemodynamic stability, size of the pregnancy mass and gestational age, serum beta-hCG level, presence or absence of fetal cardiac activity, wish for future fertility, and availability of expertise (e.g. endoscopic surgery, interventional radiology) [8, 15-17]. A comprehensive review from UKEPSS demonstrates that the success rate of expectant, medical and surgical management were 43,46 and $96 \%$, respectively [7]. The complication rates were $71 \%$ with expectant, $60 \%$ with medical and 36\% with surgical management [7].

\subsection{Expectant management}

Even though expectant management of CSEP has been described [2, 3, 15, 18], the safety of continuing pregnancy is questionable. Caution must be exercised when choosing expectant management in cases of viable CSEP as it carries a significant risk of complications, including uterine rupture, severe haemorrhage and hysterectomy $[4,18,19]$. In a systematic review of a total of 56 cases (including 44 cases with fetal cardiac activity) of CSEP managed expectantly, live births were achieved in $73 \%$ with a very high hysterectomy rate of $70 \%$ [18]. Women with viable CSEP and relatively favourable findings on imaging such as endogenic type, COS-2 on ultrasound and residual myometrial thickness of $>5 \mathrm{~mm}$ on MRI may continue with the pregnancy if they have a strong desire to do so [19]. Women need to understand that expectant management of a viable CSEP carries a significant risk of severe haemorrhage, uterine rupture, hysterectomy and severe invasive placentation $[7,15,19]$. Expectant management can be considered in those with no fetal cardiac activity and with evidence of spontaneous resolution [18-20].

\subsection{Medical management}

\subsubsection{Methotrexate}

Methotrexate (MTX) can be administered locally or systemically as single or multiple doses. Primary systemic treatment with MTX is indicated in patients with non-viable CSEP. CSEPs have been shown to respond well to systemic administration of MTX (50 mg/m²), especially in those with low serum beta-hCG levels $(<5000 \mathrm{mIU} / \mathrm{ml})$. Like in tubal ectopic pregnancy, MTX is appropriate for a woman who is pain-free and hemodynamically stable with unruptured pregnancy of $<8$ weeks' gestation. While a single dose treatment may be adequate for cases with low serum beta-hCG levels $(<5000 \mathrm{mIU} / \mathrm{ml})$, multiple doses may be necessary in those with high beta-hCG levels. 
Methotrexate can also be given directly into the gestational sac. Local MTX administration is preferable in cases with viable CSEP. Ramkrishna et al. have described their medical management protocol [21]. They use ultrasound guided intra-sac injection of $50 \mathrm{mg}$ of MTX into the gestational sac using $18 \mathrm{G}$ chorionic villus sampling needle after aspirating the content of the sac. Prior injection of $2 \mathrm{ml}$ $(30 \mathrm{mmol} / \mathrm{ml}) \mathrm{KCL}$ is used when fetal cardiac activity is present [21]. Additional dose(s) of systemic MTX is used for those cases with plateaued serum hCG level. The success rate of primary medical management (systemic/local) was $86 \%$ for CSEP in their study subjects [21].

There is a suggestion that direct injection of MTX into the sac achieves a high concentration locally and therefore interrupts the pregnancy more rapidly than a systemic administration of the drug [22]. The local injection can be done under ultrasound guidance (transabdominal or transvaginal), or direct vision through laparoscopy or hysteroscopy. A larger gauge needle is preferable to ensure better aspiration of the content of the gestational sac at the same time.

A high dose of intravenous MTX infusion, followed by oral folinic acid rescues, has also been used with a success rate of $85.7 \%$ [23].

\subsubsection{Local embryocidal injection}

Local injection of potassium chloride (or other embryocides- e.g. lignocaine [1]) can be used when there is fetal cardiac activity, or for selective embryocidal action in cases of heterotopic pregnancies [10,24]. Similar to local MTX injection, this can be done under ultrasound guidance (transabdominal or transvaginal), or direct vision through laparoscopy or hysteroscopy followed by local or systemic MTX.

\subsection{Surgical treatment}

Several surgical treatment options are described.

\subsubsection{Uterine curettage}

By definition, the gestational mass in a CSEP is not actually within the uterine cavity. Therefore, not only the trophoblastic tissue is inaccessible by the curettage but also such attempts can potentially rupture the uterine scar leading to severe haemorrhage and cause more harm [4]. A recently published meta-analysis suggested favourable outcomes (less blood loss, lower incidence of adverse events and shorter duration of hospital stay) following high-intensity focused ultrasound compared to uterine artery embolization before uterine curettage [25]. In a casecontrol study, advanced gestational age, higher serum hCG level, bigger gestational sac diameter, thinner myometrial layer and significant peritrophoblastic perfusion were associated with excessive intra-operative haemorrhage $(\geq 200 \mathrm{ml})$ during suction evacuation of CSEP [26].

\subsubsection{Hysteroscopy or laparoscopy}

Aspiration/resection of gestational contents can be accomplished either through operative hysteroscopy or laparoscopy [27-29]. The choice between laparoscopy and hysteroscopy depends on the type of CSEP. The hysteroscopic approach is more appropriate for CSEP that grows inward towards the uterine cavity (Type I), while a laparoscopy is more justified for a deeply implanted CSEP growing towards the abdominal cavity (type II). 


\subsubsection{Laparotomy}

Wedge resection of the gestation (hysterotomy) or hysterectomy should be considered in women who do not respond to conservative medical or surgical treatments, present too late or if facilities and expertise for operative endoscopy are not available. Laparotomy is mandatory when uterine rupture is confirmed or strongly suspected.

\subsection{Uterine artery embolization}

Uterine artery embolization (UAE) in combination with other treatment modalities such as MTX, dilatation and curettage (D \& C) or hysteroscopic resection has been used to treat CSEP $[15,30]$. Catheterisation of the uterine arteries is carried out through a transfemoral approach under local anaesthesia. If embolization is combined with a dose of MTX it is split between the two uterine arteries and infused via the arterial catheter. Finally, both uterine arteries are embolized with gelatin sponge particles or polyvinyl alcohol [15].

In a systematic review, UAE combined with $\mathrm{D} \& \mathrm{C}$ was highly effective with only $6.4 \%$ of cases needed additional treatment and severe complications (haemorrhage, hysterectomy) occurred in $3.4 \%$ of cases [15]. A combination of UAE, D $\& \mathrm{C}$ and hysteroscopy also resulted in a high success rate of $95.4 \%$ and a very low complication rate of $1.2 \%$. In women treated with a combination of UAE, D \& C and MTX, additional treatment was needed in $31.4 \%$ because of treatment failure and hysterectomy rate was $2.8 \%$. In comparison, between one-fourth to one-third of patient treated primarily with MTX required additional treatment [15].

\subsection{Combination}

In reality, most cases of CSEP are managed with a combination of both medical and surgical approaches [31]. Medical treatment - systemic or local, single agent or combined regiment can be used in conjunction with surgical treatment options. e.g.

- Ultrasound-guided sac aspiration, local MTX injection with or without local potassium chloride (KCL) [32]

- Local intra-sac KCL injection, systemic MTX injection [33, 34]

- Ultrasound/laparoscopic guide sac aspiration, local MTX injection, systemic MTX injection [16, 35]

- Systemic MTX, local KCL injection, laparoscopic resection [36]

- Intra-sac injection of KCL and MTX, systemic MTX, laparoscopic resection [37]

- Systemic MTX, dilatation and curettage [38, 39]

- UAE and intra-arterial MTX infusion [40]

Uterine artery embolization, bilateral uterine artery ligation, local vasopressin injection and intrauterine balloon tamponade have been used successfully as an adjunct to other conservative treatments for the prevention and control of heavy bleeding. 


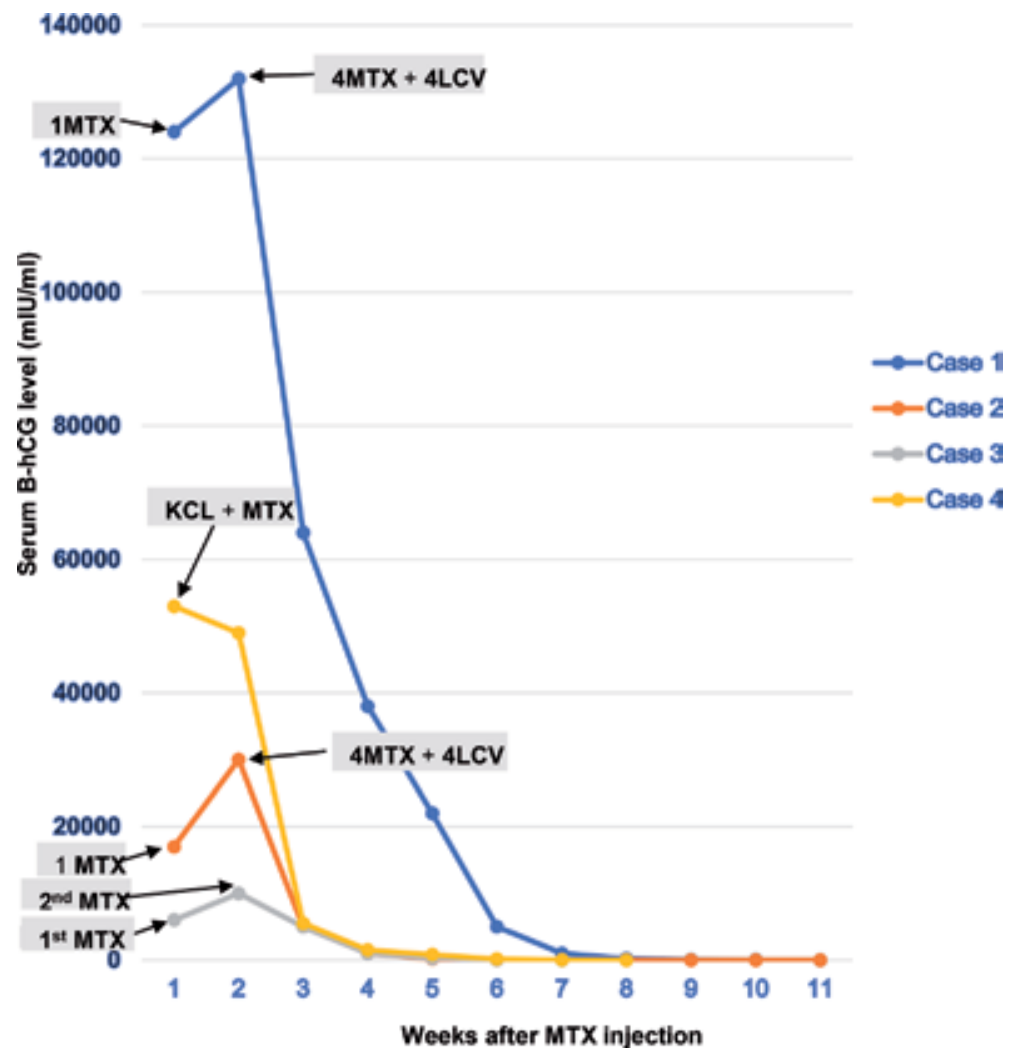

Figure 8.

Changes in weekly serum beta-hCG levels of four patients with CSEP before and after the medical treatment. The values on the curve represent the beta-hCG measurements at each given week. MTX = methotrexate; $L C V=$ Leucovorin; $K C l=$ potassium chloride [42].

\subsection{Post-treatment follow-up}

Close follow up is essential to confirm complete resolution of the CSEP. Patients need serial serum beta-hCG measurements and pelvic ultrasound until the hCG level is undetectable, and the pregnancy mass disappears, which may take several weeks (see Figure 8) [34, 41]. In general, posttreatment weekly measurement of serum beta-hCG is required to confirm resolution [42, 43]. Patients who have undergone hysterectomy do not require hCG monitoring. Serial transvaginal colour flow Doppler is useful for monitoring the response to medical treatment. It is worth to note that uterine rupture and severe maternal haemorrhage can occur despite a satisfactory drop in serum hCG levels [1]. Continuation of fetal cardiac activity or growth of the sac with rising serum hCG concentration indicates failure of medical treatment. In a tubal ectopic pregnancy, a higher failure rate of medical treatment is associated with a gestational age of $\geq 9$ weeks, a fetal pole of $>10 \mathrm{~mm}$, presence of fetal cardiac activity and a serum beta-hCG concentration $>10,000 \mathrm{mIU} / \mathrm{ml}$. However, no similar or consistent pattern could be observed for a CSEP.

\section{Recurrence and management of subsequent pregnancies}

The risk of recurrence in subsequent pregnancies is unknown. Uneventful viable intrauterine pregnancies have been reported after all modalities of conservative management of CSEP $[34,44]$. Additionally, there are case reports whereby 
patients had one or more episodes of recurrence of CSEP [44-46]. Owing to insufficient data, patients should be counselled about this uncertain risk of recurrence, and like patients with other types of ectopic pregnancies, they should be advised to avail of an early ultrasound examination in subsequent pregnancies to localise the pregnancy. Likewise, no reliable statistics exist on the safe interval between successfully treated CSEP and subsequent pregnancy. In the absence of other obstetric indication for repeat caesarean delivery, women who are pregnant following successful treatment of CSEP can consider delivering vaginally.

\section{Conclusion}

CSEP is a rare form of ectopic pregnancy but potentially associated with severe maternal complications. The incidence of CSEP is rising owing to the rise in caesarean delivery rate worldwide and widespread use of imaging in early gestation. Little is known about its exact mechanism and natural history. Ultrasonography with colour flow Doppler yields a high diagnostic accuracy. Recommendations of treatment options for CSEP are based on pieces of evidence from case reports and small case series. Therefore, treatment is individualised according to clinical presentation, availability of treatment options and expertise as well as the wish of the woman for future fertility.

\section{Conflict of interest}

The authors declare no conflict of interest.

\section{Author details}

Workineh Getaneh Tadesse

Coombe Women and Infants University Hospital, Dublin, Republic of Ireland

*Address all correspondence to: wtadesse@coombe.ie

IntechOpen

(C) 2019 The Author(s). Licensee IntechOpen. This chapter is distributed under the terms of the Creative Commons Attribution License (http://creativecommons.org/licenses/ by/3.0), which permits unrestricted use, distribution, and reproduction in any medium, provided the original work is properly cited. (cc) BY 


\section{References}

[1] Odgers HL, Taylor RA, Balendran J, Benness C, Ludlow J. Rupture of a caesarean scar ectopic pregnancy: A case report. Case Reports in Women's Health. 2019;22:e00120. DOI: 10.1016/j.crwh.2019.e00120

[2] Jayaram PM, Okunoye GO, Konje J. Caesarean scar ectopic pregnancy: Diagnostic challenges and management options. Obstetrics and Gynecology. 2017;19:13-20

[3] Elson CJ, Salim R, Potdar N, Chetty M, Ross JA, Kirk EJ. Diagnosis and management of ectopic pregnancy. BJOG: An International Journal of Obstetrics \& Gynaecology. 2016;123:e15-e55. DOI: 10.1111/1471-0528.14189

[4] Rotas MA, Haberman S, Levgur M. Caesarean scar ectopic pregnancies: Aetiology, diagnosis, and management. Obstetrics and Gynecology. 2006;107:1373-1381

[5] Osborn DA, Williams TR, Craig BM. Cesarean scar pregnancy. Journal of Ultrasound in Medicine. 2012;31:1449-1456

[6] Deepika GT, Wahi S. A rare case report of caesarean scar ectopic pregnancy. Journal of Clinical and Diagnostic Research. 2017;11(8):QD10-QD11. DOI: 10.7860/ JCDR/2017/24611.10523

[7] Harb HM, Knight M, Bottomley C, Overton C, Tobias A, Gallos ID, et al. Caesarean scar pregnancy in the UK: A national cohort study. BJOG: An International Journal of Obstetrics and Gynaecology. 2018;125(13):1663-1670. DOI: 10.1111/1471-0528.15255

[8] Gonzalez N, Tulandi T. Cesarean scar pregnancy: A systematic review. Journal of Minimally Invasive Gynecology. 2017;24:731-738
[9] Ouyang Y, Li X, Yi Y, Gong F, Lin G, Lu G. First-trimester diagnosis and management of caesarean scar pregnancies after in vitro fertilisation - embryo transfer: A retrospective clinical analysis of 12 cases. Reproductive Biology and Endocrinology. 2015;13:126. DOI: 10.1186/s12958-015-0120-2

[10] Litwicka K, Greco E, Prefumo F, Fratelli N, Scarselli F, Ferrero S, et al. Successful management of a triplet heterotopic caesarean scar pregnancy after in vitro fertilisation-embryo transfer. Fertility and Sterility. 2011;95(1):291.e1-291.e3

[11] Kim DJ, Welch M, Kendall JL. A case of cesarean scar ectopic: A rare but important form of ectopic pregnancy. Critical Ultrasound Journal. 2011;3(1):55-57. DOI: 10.1007/ s13089-011-0064-5

[12] Cali G, Forlani F, Timor-Tritsch IE, Palacios-Jaraquemade J, Minneci G, D'Antonio F. Natural history of caesarean scar pregnancy on prenatal ultrasound: The crossover sign. Ultrasound in Obstetrics \& Gynecology. 2017;50(1):100-104

[13] Collins K, Kothari A. Catastrophic consequences of caesarean scar pregnancy missed on ultrasound. Australasian Journal of Ultrasound in Medicine. 2015;18(4):150-156. DOI: 10.1002/j.2205-0140.2015.tb00222.x

[14] Samal SK, Rathod S. Cervical ectopic pregnancy. Journal of Natural Science, Biology and Medicine. 2015;6:257-260

[15] Petersen KB, Hoffmann E, Larsen CR, Nielsen HS. Caesarean scar pregnancy: A systematic review of treatment studies. Fertility and Sterility. 2016;105:958-967

[16] Tadesse WG, Von Bunau G. A combination of surgery and 
methotrexate for successful treatment of a caesarean scar pregnancy. Irish Medical Journal. 2018;111(6):774

[17] Tahaoglu AE, Dogan Y, Bakir MS, Peker N, Cavus Y, Togrul C, et al. A single centre's experience of caesarean scar pregnancy and proposal of a management algorithm. Journal of Obstetrics and Gynaecology. 2019;39(2):259-264. DOI: 10.1080/01443615.2018.1499714

[18] Jayaram P, Okunoye G, Al Ibrahim AA, Ghani R, Kalache K. Expectant management of caesarean scar ectopic pregnancy: A systematic review. Journal of Perinatal Medicine. 2018;46(4):365-372. DOI: 10.1515/ jpm-2017-0189

[19] Calì G, Timor-Tritsch IE, Palacios-JaraquemadaJ, MonteaugudoA, Buca D, Forlani F, et al. Outcome of cesarean scar pregnancy managed expectantly: Systematic review and meta-analysis. Ultrasound in Obstetrics \& Gynecology. 2018;51(2):169-175

[20] Jabeen K, Karuppaswamy J. Nonsurgical management of caesarean scar ectopic pregnancy - a five-year experience. Journal of Obstetrics and Gynaecology. 2018;38(8):1121-1127. DOI: 10.1080/01443615.2018.1451986

[21] Ramkrishna J, Kan GR, Reidy KL, Ang WC, Palma-Dias R. Comparison of management regimens following ultrasound diagnosis of non-tubal ectopic pregnancies: A retrospective cohort study. BJOG: An International Journal of Obstetrics \& Gynaecology. 2018;125:567-575

[22] Uludag SZ, Kutuk MS, Ak M, Ozgun MT, Dolanbay M, Aygen EM, et al. Comparison of systemic and local methotrexate treatments in caesarean scar pregnancies: Time to change conventional treatment and follow-up protocols. European
Journal of Obstetrics, Gynecology, and Reproductive Biology. 2016;206:131-135

[23] Tanaka K, Coghill E, Ballard E, Sekar R, Amoako A, Khalil A, et al. Management of caesarean scar pregnancy with high dose intravenous methotrexate infusion therapy: 10-year experience at a single tertiary centre. European Journal of Obstetrics, Gynecology, and Reproductive Biology. 2019;237:28-32. DOI: 10.1016/j.ejogrb.2019.04.008

[24] Paoletti D. A heterotopic pregnancy involving a caesarean section scar. Australasian Journal of Ultrasound in Medicine. 2011;14(3):34-35

[25] Xiao X, Feng Z, Li T, Zhang S, Wang W. Comparing the efficacy and safety of high-intensity focused ultrasound and uterine artery embolisation in caesarean scar pregnancy: A meta-analysis. Advances in Therapy. 2019;36(6):1314-1325. DOI: 10.1007/s12325 -019-00959-w

[26] Wang Q, Ma H, Peng H, He L, Bian C, Zhao X. Risk factors for intraoperative haemorrhage and bleeding risk scoring system for caesarean scar pregnancy: A case-control study. European Journal of Obstetrics, Gynecology, and Reproductive Biology. 2015;195:141-145. DOI: 10.1016/j. ejogrb.2015.06.023

[27] Fuchs N, Manoucheri E, Verbaan M, Einarsson JI. Laparoscopic management of extrauterine pregnancy in caesarean section scar: Description of a surgical technique and review of the literature. BJOG: An International Journal of Obstetrics and Gynaecology. 2015;122(1):137-140

[28] He M, Chen MH, Xie HZ, Yao SZ, Zhu B, Feng LP, et al. Transvaginal removal of ectopic pregnancy tissue and repair of uterine defect for caesarean scar pregnancy. BJOG: An International Journal of Obstetrics and Gynaecology. 2011;118(9):1136-1139 
[29] Yang Q, Piao S, Wang G, Wang Y, Liu C. Hysteroscopic surgery of ectopic pregnancy in the caesarean section scar. Journal of Minimally Invasive Gynecology. 2009;16(4):432-436

[30] Maheux-Lacroix S, Li F, Bujold E, Nesbitt-Hawes E, Deans R, Abbott J. Caesarean scar pregnancy: As systematic review of treatment options. Journal of Minimally Invasive Gynecology. 2017;24(6):915-925

[31] Alalade AO, Smith FJE, Kendall CE, Odejinmi F. Evidencebased management of non-tubal ectopic pregnancies. Journal of Obstetrics and Gynaecology. 2017;37(8):982-991. DOI: 10.1080/01443615.2017.1323852

[32] Tanigaki S, Nagata C, Ueno K, Ozawa N, Nagaoka S, Tanaka K, et al. Successful treatment of caesarean scar pregnancies by local treatment only. Obstetrics and Gynecology International. 2017;2017:9543570. DOI: $10.1155 / 2017 / 9543570$

[33] Gupta M, Kriplani A, Mahey R, Kriplani I. Successful management of caesarean scar live ectopic pregnancies with local KCL and systemic methotrexate. BML Case Reports 2017; 2017.pii: bcr-2017-221844. doi: $10.1136 /$ bcr-2017-221844

[34] Piejani R, Bayani L, Shirazi M. Successful local and systemic medical treatment of caesarean scar pregnancy and a subsequent term pregnancy after treatment: A case series. Iranian Journal of Reproductive Medicine. 2015;13(7):445-450

[35] Seow KM, Wang PH, Huang LW, Hwang JL. Transvaginal sono-guided aspiration of gestational sac concurrent with a local methotrexate injection for the treatment of unruptured caesarean scar pregnancy. Archives of Gynecology and Obstetrics. 2013;288(2):361-366
[36] Mahgoub S, Gabriele V, Faller E, Langer B, Wattiez A, Lecointre L, et.al. Caesarean scar ectopic pregnancy: Laparoscopic resection and total scar dehiscence repair. Journal of Minimally Invasive Gynecology. 2018;25(2):297-298

[37] Ades A, Parghi S. Laparoscopic resection of caesarean scar ectopic pregnancy. Journal of Minimally Invasive Gynecology. 2017;24(4):533-535

[38] Mahapatro AK, Shankar K, Varma T. Caesarean scar ectopic pregnancy: Reports of two cases. Journal of Clinical and Diagnostic Research. 2016;10(5):QD05-QD06

[39] Datta S, Jha C. Suction evacuation with methotrexate as a successful treatment modality for caesarean scar pregnancies: Case series. Sultan Qaboos University Medical Journal. 2015;15(4):e539-e545. DOI: 10.18295/ squmj.2015.15.04.017

[40] Elmokadem AH, Abdel-Wahad RM, El-Zayadi AA, Elrakhawy MM. Uterine artery embolisation and methotrexate infusion as sole management of caesarean scar and cervical ectopic pregnancies: A single- centre experience and literature review. Canadian Association of Radiologists Journal 2019; pii: S0846-5371(18)30210-9. doi: 10.1016/j.carj.2018.12.002

[41] Ko JK, Li RH, Cheung VY. Caesarean scar pregnancy: A 10-year experience. The Australian \& New Zealand Journal of Obstetrics \& Gynaecology. 2015;55(1):64-69. DOI: 10.1111/ ajo.12273

[42] Boza A, Boza B, Api M. Cesarean scar pregnancy managed with conservative treatment. Iranian Journal of Medical Sciences. 2016;41(5):450-455

[43] Qian ZD, Weng Y, Du YJ, Wang CF, Huang LL. Management of persistent caesarean scar pregnancy after curettage 
treatment failure. BMC Pregnancy and Childbirth. 2017;17(1):208. DOI:

10.1186/s12884-017-1395-4

[44] Gao L, Huang Z, Zhang X, Zhou N, Huang X, Wang X. Reproductive outcomes following cesarean scar pregnancy - a case series and review of the literature. European Journal of Obstetrics, Gynecology, and Reproductive Biology. 2016;200:102-107

[45] Holland MG, Bienstock JL. Recurrent ectopic pregnancy in a cesarean scar. Obstetrics and Gynecology. 2008;111(2 Pt 2):541-545

[46] Bennett TA, Morgan J, Timor-Tritsch IE, Dolin C, Dziadosz M, Tsai M. Fifth recurrent cesarean scar pregnancy: Observations of a case and historical perspective. Ultrasound in Obstetrics \& Gynecology.

2017;50(5):658-660 



\title{
A Minimally Invasive Hemostatic Strategy for Cesarean Scar Pregnancy and Cervical Pregnancy
}

\author{
Satoru Takeda, Jun Takeda, Takashi Yorifuji \\ and Taro Koshiishi
}

\begin{abstract}
Cesarean scar pregnancy (CSP) and cervical pregnancy are categorized as non-tubal ectopic pregnancy, because these are associated with a high burden of maternal and fetal morbidity including early uterine rupture, prevalence of placenta previa accrete spectrum, massive hemorrhage, and hysterectomy. Although management methods vary according to the week of gestation, recent reviews and reports support an interventional or a combination of surgical and medical approaches for treatment of unruptured CSP and cervical pregnancy rather than medical approach alone. In cases of massive hemorrhage, pressure hemostasis using balloon tamponade should first be performed. If such hemostasis proves to be ineffective, surgical excision or transcatheter arterial embolization (TAE) should be selected next. TAE reportedly achieves a high hemostasis rate. However, complications such as subsequent endometrial hypoplasia, menstruation disorder, infertility, placenta accreta, and uterine rupture have been reported, even in cases that have undergone successful hemostasis with TAE using an absorbable embolus. Recently, a minimally invasive hemostatic strategy in obstetrics, which aims to preserve uterine function and enhance the safety of subsequent pregnancies, has been developed. Therefore, we should reconsider uterus-preserving hemostatic strategies for critical hemorrhage and management of non-tubal ectopic pregnancy under these circumstances by using safe and minimally invasive treatment modalities.
\end{abstract}

Keywords: arterial embolization, balloon tamponade, cervical pregnancy, cesarean scar pregnancy, massive hemorrhage, methotrexate

\section{Introduction}

Cesarean scar pregnancy (CSP) and cervical pregnancy are categorized as non-tubal ectopic pregnancy and may cause massive hemorrhage, uterine rupture, and hysterectomy. In CSP and cervical pregnancy cases, it is possible that the location of the gestational sac (GS) may lead to a misdiagnosis of abortion. Another possibility is a failure to detect CSP until massive hemorrhage occurs following curettage. Therefore, diagnosis and treatment strategies in early pregnancy are 
important. Delay in the diagnosis and management may lead to massive hemorrhage, which is difficult to control. This complication can be treated with life-saving hysterectomy or uterine arterial embolization, which requires massive blood transfusion [1]. Management based on the pathophysiology of the implantation site that has a rich blood supply is essential. Although management methods vary according to the week of gestation, typical strategies include surgical treatment, administration of drugs such as methotrexate (MTX), and combinations of these treatments. In addition, hemostatic procedures vary and include balloon tamponade, transcatheter arterial embolization (TAE), and surgical treatments such as curettage, evacuation, wedge resection, and hystero-resectoscopy, for control and prevention of hemorrhage.

In the field of obstetrics, TAE is known to be highly effective in controlling uterine hemorrhage and hematoma. This procedure reportedly achieves a high hemostasis rate, and thus, the frequency of hysterectomy has sharply decreased [2, 3]. However, complications such as subsequent endometrial hypoplasia, menstruation disorder, infertility, pregnancy loss, placenta accreta, and uterine rupture have been reported, even in cases that have undergone successful hemostasis with TAE using an absorbable embolus [4-6]. Recently, a minimally invasive hemostatic strategy in obstetrics, which aims to preserve uterine function and enhance the safety of subsequent pregnancies, has been developed $[2,7]$. Therefore, we should reconsider uterus-preserving hemostatic strategies for critical hemorrhage and management of non-tubal ectopic pregnancy under these circumstances by using safe and minimally invasive treatment modalities. We herein discuss how to select the optimal hemostatic strategy and its management based on literatures and our experiences of non-tubal ectopic pregnancy.

\section{Pathology and frequency}

When the uterus is observed by ultrasonography more than 3 months after cesarean section (by transverse incision in the lower uterine segment), endometrial defect and defect or thinning of the myometrium at the site of the uterine incision are recognized as a triangular echo-free space at a frequency of approximately $50 \%$ [8].

Implantation at this site is considered to be the origin of CSP [9]. Delay in the diagnosis and treatment of CSP may cause uterine rupture and massive hemorrhage, resulting in hysterectomy or massive blood transfusion in serious cases. In the case of CSP, the site of villous anchoring is the isthmus of the uterus or the cervical canal where the endometrium and cervical mucosa are thinner than in the uterine body. Therefore, chorionic villi are likely to penetrate into the myometrium, inducing conditions similar to placenta increta and placenta percreta. The clinical picture and course vary according to the site of villous anchoring [1].

It has been reported that the frequency of CSP is $0.04-0.19 \%$ among all pregnancies, $0.15 \%$ among those with prior cesarean section, and $6.1 \%$ among ectopic pregnancies associated with prior cesarean section [10]. Recently, a national cohort study showed that the estimated incidence of CSP was $0.015 \%$ in the UK [11]. It is speculated that the incidence of CSP may increase in the future as the rate of cesarean section rises.

\section{Diagnosis}

Chorionic villi are smaller and penetrate less deeply into the myometrium in the early stages of pregnancy. Therefore, they are considered to be easier 
to remove, and it is also believed that the uterus is more likely to be preserved, in early pregnancy. After 9 or 10 weeks of gestation, the placenta invades the myometrium more deeply, and there is abundant blood flow into the placental bed, increasing the risk of massive hemorrhage. The risk of uterine rupture and placental invasion into the bladder is also increased. When accurately diagnosing CSP, it is important to observe the boundary area between the uterine body and the cervix in early pregnancy. The decisive factors in making a diagnosis of CSP are the presence of a gestational sac (GS) in that area and villous adhesion to the anterior wall (Table 1) $[1,8,12-14]$. Transvaginal ultrasonography is useful for identifying the implantation of chorion frondosum in the scar area (Table 2, Figure 1); the reported sensitivity of this modality is $84.6 \%$ [14]. The combined use of color Doppler ultrasonography allows clinicians to estimate the viability of gestational tissue $[10,13]$, providing useful information for selection of the most appropriate

1. Diagnosis using transvaginal ultrasonography and contrast-enhanced magnetic resonance imaging

- Evaluation of the implantation site

- Evaluation of blood flow around the villous tissue invading the scar area (Figures 2 and 3)

- Evaluation of thinning of the scar area, presence/absence of continuity of the myometrium, and presence/absence of villous invasion into the bladder

2. Evaluation of the viability of the villi

- Blood hCG level

- Color Doppler ultrasonography

3. Presence/absence of vaginal bleeding

Table 1.

Key points in diagnosing cesarean scar pregnancy.

1. There is no gestational sac in the uterine cavity.

2. In the sagittal view of the uterus, thinning and a wedge-shaped defect of the anterior wall myometrium are detectable in the wound area of the previous cesarean section, and the gestational sac is present in that area.

3. Villi (high echoic area) are observed on the bladder side of the anterior wall of the uterus.

4. Color Doppler ultrasonography shows blood flow in the gestational sac and areas around the scar.

Table 2.

Ultrasonographic findings of cesarean scar pregnancy.

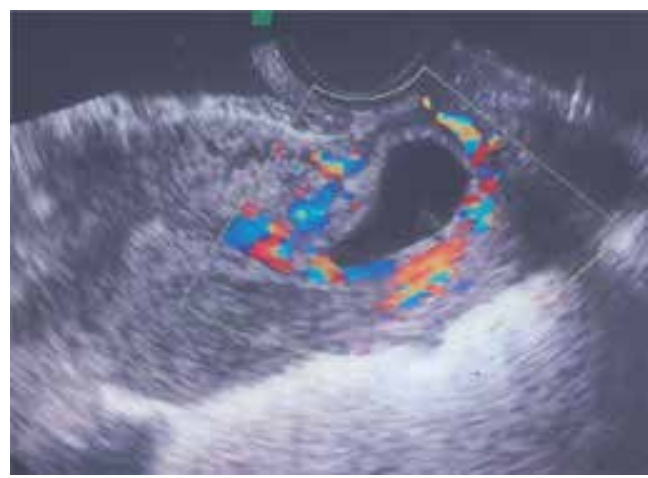

Figure 1.

Findings of transvaginal color Doppler ultrasonography (produced with permission from [1]). The gestational sac accompanied by blood flow in the surrounding tissues is seen near the scar of the previous cesarean section (endogenic type). There is no gestational sac in the uterine cavity. 


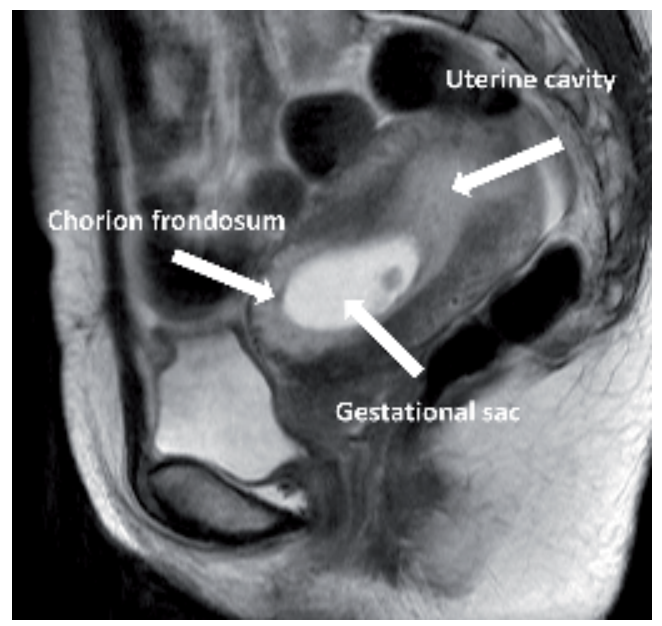

Figure 2.

Findings of T2-weighted magnetic resonance imaging (produced with permission from [1]). This is a case of cesarean scar pregnancy with positive fetal heartbeats at 9 weeks of gestation. The chorion frondosum is bounded on the bladder and extends into the thinned myometrium of the scar.

treatment method. The descending GS in the event of an inevitable abortion and GS in CSP can be distinguished by the presence/absence of blood flow in the villous area [1]. Magnetic resonance imaging (MRI) is also useful for determining the thickness of the myometrium in the wound site and the depth of villous invasion into the bladder, as well as for identifying nutrient vessels (Figure 2) [1]. When invasion into the bladder is suspected in the middle stages of pregnancy or thereafter, confirmation by cystoscopy is required.

\section{Management of cesarean scar pregnancy (CSP)}

Although expectant management of CSP with positive fetal heart activity may still be a choice $[11,15]$, termination of CSP and cervical pregnancy should be offered to these women and families as one of the therapeutic options. The basic policy for the management of CSP and cervical pregnancy is termination of pregnancy and preservation of the uterus, because these are associated with a high burden of maternal and fetal morbidity including complicated miscarriage, early uterine rupture, prevalence of placenta previa accreta spectrum, massive hemorrhage, hysterectomy, maternal and fetal death, etc. The method of treatment should be selected according to the gestational week, presence/absence of fetal heart beats, the blood hCG level, and abundance of the blood supply adjacent to villi or the gestational sac.

Chemotherapeutic drugs such as methotrexate (MTX) are administered systemically or locally, with or without potassium chloride ( $\mathrm{KCl})$, to avoid surgical treatment for CSP [16-19]. However, achieving the desired healing with drug treatment alone can be time-consuming, and hemorrhage or infection may occur concomitantly during the process of treatment. MTX treatment alone as the first-line therapy showed low success rate. In addition, adverse reactions to drugs, such as stomatitis and leucocytopenia, may also occur. Recent reviews and reports support an interventional or a combination of surgical and medical approaches rather than medical approach alone [20-24]. In a national cohort study, surgical management appears to be associated with a high success rate, low complication rate, and short posttreatment follow-up [11]. 

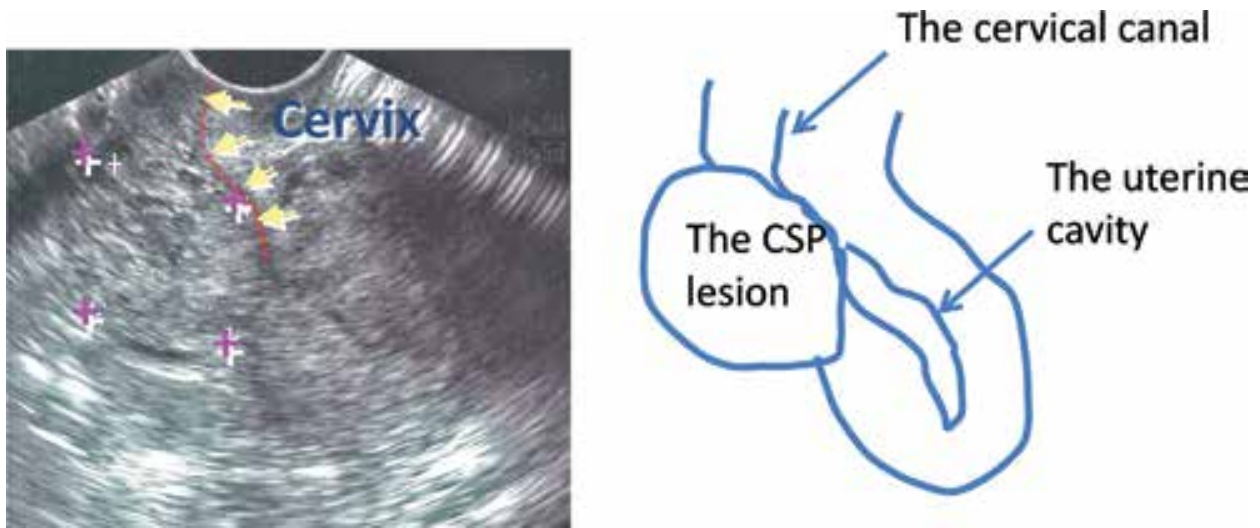

Figure 3.

Protrusion of the cesarean scar pregnancy lesion toward the bladder (produced with permission from [1]). The cesarean scar pregnancy lesion is seen protruding toward the outside of the uterus (exogenic type) and forming a mass.

If there is a CSP mass protruded toward outside of the uterus (exogenic type) (Figure 3), or a mass attached to defect or thinning $(<2 \mathrm{~mm})$ of lower uterine myometrium, the lesion may be removed laparoscopically or by laparotomy and then sutured [25-27], because these types of CSPs could be complicated with uterine rupture and bleeding early in pregnancy. But evidence-based treatment still remains unclear in these cases.

\subsection{Surgical approach}

\subsubsection{Curettage}

Curettage or evacuation allows preservation of fertility, can be performed under intravenous or spinal anesthesia, and is minimally invasive $[1,18,20,22-25$, $27,28]$. Curettage or evacuation may be performed under ultrasound guide after administration of MTX and/or $\mathrm{KCl}$. In addition, it requires only a short period of hospitalization in successful cases. On the other hand, there is the risk of massive hemorrhage or uterine perforation during surgery as well as complications such as uterine rupture and residual tissue. Additional treatment such as balloon compression hemostasis or TAE may be required to deal with bleeding [27].

It has been reported that massive hemorrhage, difficult to control, occurred after curettage in patients who had a low hCG level but had abundant blood flows in the lesion, prompting caution in the management of such patients [1]. This is because neovascularity may persist even when the hCG level is decreased after villi have been devitalized by chemotherapy or dilation and curettage (D\&C).

\subsubsection{Other surgical procedures}

In patients with massive hemorrhage after D\&C or with CSP diagnosed after massive hemorrhage, priority is given to hemostasis. Therefore, CSP is removed by transvaginal resection [26] or laparotomic resection [29], and uterine artery ligation [30], cervical suture [31], or TAE is performed. When deep villous invasion is predicted in view of the gestational week, the fertilized ovum should be removed by abdominal resection and suture in cases with wound rupture or dehiscence or subserous protrusion of the lesion.

Along with recent advancements in laparoscopic techniques, cases undergoing less invasive laparoscopic or hysteroscopic resection of the lesion have been 
reported [14, 18, 20, 22, 23]. Surgical resection of the lesion and wound repair (wedge resection and repair) is reportedly less likely to require additional treatment and is highly effective for the prevention of recurrence. However, at present, data on the scientific basis of treatment and recurrence of CSP are lacking.

\section{Management of cervical pregnancy}

Several case reports and literatures support surgical therapy alone or in combination with MTX and/or mifepristone rather than medical therapy alone for treatment of cervical pregnancy as the same as those of CSP [21, 32-35]. Uterine artery embolization for treatment and prevention of hemorrhage during curettage or evacuation is also effective to preserve the uterus [36-39].

To control bleeding during curettage and evacuation, hemostatic techniques such as hemostatic clamps or cervical cerclage to block cervical blood supply and balloon tamponade by Foley catheter or a cervical ripening balloon were reported [40-44]. Recently, more minimally invasive total hysteroscopic treatment for cervical pregnancy and also conservative treatment, using a cervical ripening double-balloon catheter alone, were reported $[44,45]$. These seem to be an effective, safe, and minimally invasive and single promising treatment without any medical and surgical treatment. These individual managements have to be validated on a large patient population.

\section{Control of bleeding}

Massive hemorrhage reportedly occurs in some cases after evacuation of the uterus $\mathrm{D} \& \mathrm{C}$ has on occasion been performed without an accurate diagnosis. In such cases, balloon tamponade, TAE, and surgical treatment are effective management strategies. If balloon tamponade or TAE is successful in controlling hemorrhage, conservative treatment is also a feasible option. If bleeding is under control, there are several possible treatment options for preservation of the uterus. These options have their own advantages and disadvantages, and selection of the most appropriate treatment varies according to the facility and the time of day. Therefore, the treatments should be tailored to individual patients based on the situation and their wishes regarding fertility.

In cases experiencing massive hemorrhage, pressure hemostasis using balloon tamponade should first be performed. If such hemostasis proves to be ineffective, surgical excision or TAE should be selected next. When performing cesarean scar resection, the right and left uterine arteries are to be clamped.

\subsection{Balloon tamponade}

This is a hemostatic technique used for overall uterine hemorrhage in the puerperal period including atonic bleeding, incomplete cervical laceration, crush syndrome, and bleeding from the separation surface of placenta previa. Balloon tamponade is also effective and useful for uterine hemorrhage from CSP and cervical pregnancy (Figure 4) [46]. In an emergency, insertion and placement of the balloon are technically easy and can be performed expeditiously; this technique is simple and minimally invasive. It is also possible to determine within a short period of time whether hemostasis can be achieved by tamponade alone (tamponade test) [47]. Even when switching to TAE is necessary, the radiology department does not need to be consulted, and the amount of bleeding during preparation for TAE can be decreased. This technique is also useful for temporary hemostasis when a patient must be transferred to an advanced medical facility. 


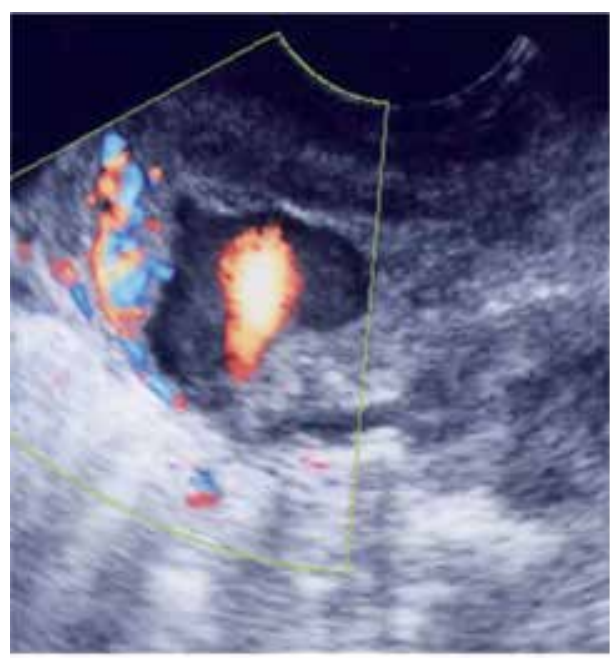

A

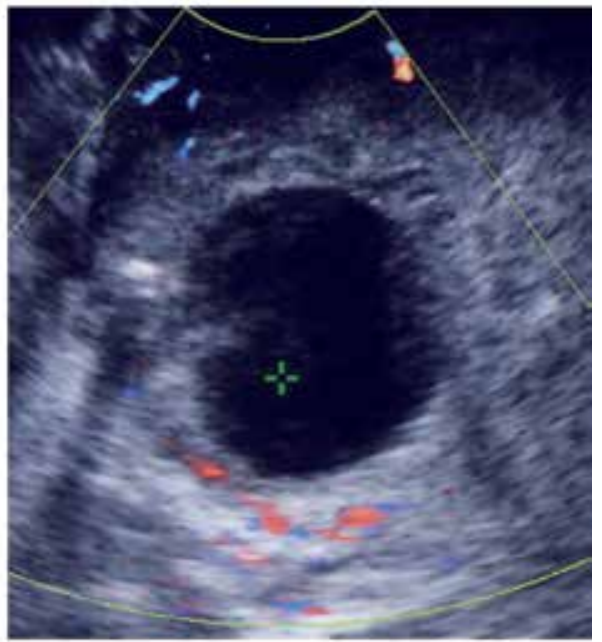

B

Figure 4.

Compression hemostasis by balloon tamponade (produced with permission from [1]). (A) In a case with cesarean scar pregnancy, after dilation and curettage, a hematoma (pooling of blood) was observed in the wound area, which prominently showed blood spurting out into its cavity. This case is the same as the one at 9 weeks of gestation in Figure 2. (B) The blood flow disappears after inflation with a Minimetro ${ }^{\circledR}$. A balloon should be inserted from the uterine os guided by ultrasound. Physiological saline (20-30 $\mathrm{mL}$ ) is then infused into the balloon. The balloon should be gently pulled to confirm that it will not easily prolapse into the vaginal cavity and that bleeding from the uterine os has been halted. Recently, the bleeding point after dilation and curettage could be precisely detected by contrast-enhanced ultrasonography, and a balloon could be inflated at the bleeding point until bleeding stops.

The balloon should be gently pulled to confirm that it would not easily prolapse into the vaginal cavity and that bleeding from the cervical os has been halted. Long gauze should be retained in the vaginal cavity to prevent prolapse of the balloon. A urethral catheter should be placed in the bladder to prevent urinary retention. If there is no massive hemorrhage that would surpass the absorption capacity of the retained gauze, the balloon is to be removed in $24-48 \mathrm{~h}$. The presence of uncontrollable bleeding immediately after the insertion should be deemed a negative result of the tamponade test, and the procedure is then switched to TAE or laparotomic hemostasis without hesitation.

\subsection{Transcatheter arterial embolization (TAE)}

If bleeding is not controllable by the procedures described above, arterial embolization should be performed without hesitation. TAE is advantageous in that it allows embolization of not only the artery in question but also anastomotic branches while confirming the bleeding point. The rates of hemostasis achievement are reportedly in the range of $89-97 \%[1,3]$.

It is known that, in CSP cases, abnormal growth and anastomosis of inflow vessels occur when the shaggy chorion grows not in the uterine body but in the vicinity of the site of entry into the uterine artery; this causes pathological features different from those associated with the uterine blood flow distribution in normal pregnancy. Therefore, unlike the conventional uterine arterial blood flow, vascular anastomoses are not restricted to those derived from the internal iliac artery. Various anastomoses involving the external iliac artery, lumbar artery, inferior mesenteric artery, sacral artery, superior gluteal artery upstream of the internal iliac artery system, and the external iliac artery system are observed. 
Rebleeding may occur due to recanalization or insufficient embolization, and it is difficult to control bleeding when there is abundant blood flow from the external iliac artery region. Therefore, attention should be paid to the patient's general condition after implementation of TAE. In most cases, bleeding can be controlled by TAE. If TAE is ineffective, or if bleeding persists, it is recommended that the aforementioned balloon tamponade be added to the management. Gelatin sponge is usually used as a temporary solid embolic substance lasting for several days to about 2 weeks. In addition, metal coils as permanent solid embolic substances and N-butyl-2-cyanoacrylate as a permanent liquid embolic substance are also available. The incidence rate of adverse reactions is $6-7.8 \%$. Fever is the most frequent untoward effect. Other adverse reactions include endometrial necrosis, adhesion, myometrial necrosis, ovarian insufficiency, bladder necrosis, gluteal muscle necrosis, and pelvic suppuration $[1,3]$. Pregnancy after TAE for postpartum hemorrhage may be associated with increased risk of obstetric hemorrhage due to placenta accreta spectrum. Therefore, precautions in perinatal management are required in managing the subsequent pregnancy.

\subsection{Surgical hemostasis}

If bleeding is uncontrollable employing the procedures described above, laparotomic hemostasis should be selected as the last resort. Since TAE became available, application of this technique has mostly been limited to cases of uterine rupture in the affected area. Hysterectomy, ligation of the uterine artery, or wedge resection and repair of the lesion in the scar should be performed. Prompt selection of the optimal procedure, taking into consideration the amount of bleeding, size of the lesion, whether the patient desires fertility preservation, etc., is necessary. Patients with indications for these procedures often have concomitant obstetric coagulopathy. Therefore, sufficient supplementation of coagulation factors is also essential prior to surgery $[2,7]$.

\subsection{Treatment of coagulopathy}

In cases of massive hemorrhage, patients must be kept in good systemic condition, and local hemostasis must be achieved while paying attention to the possible occurrence of coagulopathy under monitoring of fibrinogen levels as point-of-care testing in order to perform early diagnosis and treatment of coagulopathy [7]. When coagulopathy is present, local hemostasis, such as balloon tamponade, surgical sutures, and TAE, is difficult to achieve because of hemorrhagic tendency. In these cases of coagulopathy, the blood fibrinogen level is often $<100 \mathrm{mg} / \mathrm{dL}$. Therefore, the treatment of coagulopathy should be performed by combined administration of concentrated coagulation factors (fibrinogen concentrate and cryoprecipitate) and fresh-frozen plasma promptly to obtain a blood fibrinogen level of at least $150-200 \mathrm{mg} / \mathrm{dL}[7,48]$. If coagulopathy is eliminated, the conventional hemostatic procedures become effective.

\section{A minimally invasive hemostatic strategy in CSP and cervical pregnancy}

Curettage alone is reportedly not a suitable first-choice procedure because using curettage only may lead to serious complications such as massive hemorrhage or uterine rupture, necessitating additional treatment in $76.2 \%(16 / 21)$ of patients [1]. On the other hand, the methotrexate (MTX) monotherapy is time-consuming 
in terms of achieving cure and may be accompanied by hemorrhage or infection. Reduction of the chorionic tissue by MTX therapy to decrease blood flow, followed by curettage or laparotomy, rather than MTX or curettage alone, achieves a higher cure rate and is associated with fewer complications such as hemorrhage, infection, and sepsis.

For the management of CSP and cervical pregnancy in the first trimester, we first perform ultrasonography to confirm the implantation site of the gestational sac, observe the status and thickness of the myometrium in surrounding areas and the presence/absence of fetal heart beats, and determine the gestational week. At the same time, we measure the blood hCG level and evaluate blood flow around the gestational sac by Doppler ultrasonography. Changes in the blood hCG levels and the status of blood flow are useful for judging the viability of chorionic villi and the efficacy of estimating chemotherapy. If there is abundant blood flow in the myometrium as well as in the tissues surrounding the gestational sac, villous invasion site on the myometrium is suspected. In such cases, implementation of D\&C alone is expected to cause massive hemorrhage.

When there are fetal heart beats, potassium chloride $(\mathrm{KCl})$ is administered directly to the fetus to cause cessation of the heart beating. MTX is administered systemically ( $1 \mathrm{mg} / \mathrm{kg}$ body weight) or by local injection to the villous area at the same time. Subsequently, MTX is administered systemically every 7-10 days. In patients with high blood levels of hCG, MTX therapy used to be performed to decrease the hCG level to a target of $20,000 \mathrm{mIU} / \mathrm{mL}$, or even lower, followed by TAE and curettage. The rates of blood transfusion and/or blood loss of $>2500 \mathrm{~mL}$ in these combined treatment (MTX + TAE + D\&C) were significantly decreased, compared with those in $\mathrm{D} \& \mathrm{C}$ alone, 9.5 and $83.3 \%$ respectively.

The rate of additional surgical treatment, such as wedge resection or hysterectomy, in combined treatment, was none, compared with D\&C alone, 0 and 50.0\%, respectively. There were no complications such as uterine rupture, postoperative infections, menstrual abnormalities, and ovarian dysfunction in D\&C alone and combined treatment. These combined treatments yielded satisfactory results with a decreased volume of blood loss, no additional surgical treatment, and a high cure rate, compared with D\&C alone (Figure 5, Tables 3 and 4).

Currently, prophylactic TAE before D\&C is avoided whenever possible, with the aim of preventing short-term complications and uterine rupture in the subsequent pregnancy, and also shortening hospital stay. A target blood hCG levels after MTX is also changed to $<40,000 \mathrm{mIU} / \mathrm{mL}$ for adverse effects of MTX. Then, ultrasoundguided D\&C to evacuate the gestational sac is performed a week later after MTX therapy. Bleeding point during D\&C could be found by contrast-enhanced ultrasonography, and a cervical ripening miniballoon (Minimetro ${ }^{\circledR}$ ) could be inserted at the bleeding point and inflated until bleeding stops [46, 49]. If bleeding continues, TAE or laparoscopic wedge resection would be performed as soon as possible. Nine patients with CSP (6-12 weeks gestation) and one (7 weeks gestation) with cervical pregnancy were treated under new management. Seven cases were successfully treated with D\&C and balloon tamponade after MTX. Additional treatments were needed in three cases for bleeding. This combined therapy resulted in all complete cure and no additional surgical therapy without complications.

Moreover, if early detection of CSP is possible, more minimally invasive treatments might be another option of treatment such as double balloon conservative therapy or hysteroscopic resection without MTX therapy. We also experienced one case with CSP to be successfully treated after cessation of fetal heart beats by local injection of $\mathrm{KCl}$. She selected conservative therapy without chemotherapy and surgical therapy and then was just followed up in outpatient clinic. It took 6 months to be cured completely, but there were no complications and no bleeding. Even in 


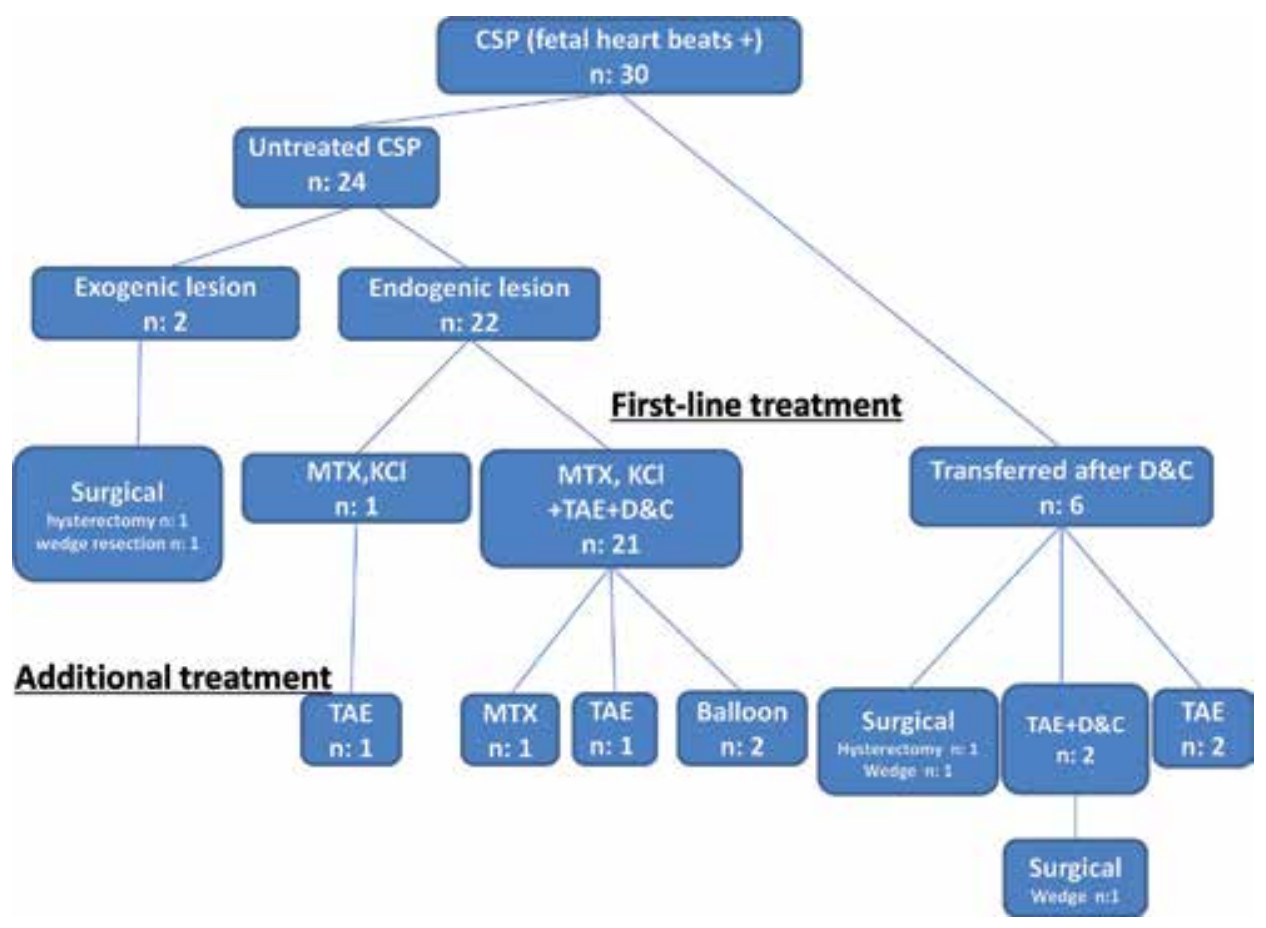

Figure 5.

Treatment results of cesarean scar pregnancy with fetal heart beats under uterus-preserving management. Thirty patients diagnosed with cesarean scar pregnancy with positive fetal heartbeat in the first trimester were treated at Saitama Medical Center and Juntendo University Hospital from 1998 to 2010. The average maternal age was $33.3 \pm 5.8$ years (mean $\pm S D$ ), and average gestational age on admission was $6.6 \pm 1.5$ weeks of gestation. The average hCG level on admission was $29,534 \pm 26,284 \mathrm{mIU} / \mathrm{mL}$, and its level on dilation and curettage was 19,995 $\pm 24,765 \mathrm{mIU} / \mathrm{mL}$. Twenty-one patients were treated under our uterus-preserving management policy and six patients were transferred to our hospital due to uncontrollable hemorrhage after dilation and curettage. This management strategy yielded satisfactory results with a decreased volume of blood loss and a high cure rate.

\begin{tabular}{|c|c|c|c|}
\hline Characteristic & $\begin{array}{c}\text { D\&C alone } \\
(n: 6)\end{array}$ & MTX + TAE + D\&C (n:21) & $P$-value \\
\hline \multirow[t]{2}{*}{ Age (years) } & $34.5+3.0$ & $34.0+5.8$ & 0.84 \\
\hline & $(32-39)$ & $(23-42)$ & \\
\hline \multirow[t]{2}{*}{ Number of previous CS (n) } & $1.5+0.8$ & $1.3+0.6$ & 0.47 \\
\hline & $(1-3)$ & $(1-3)$ & \\
\hline \multirow[t]{2}{*}{ Gestational age at diagnosis (weeks) } & $7.8+2.4$ & $6.3+1.1$ & 0.19 \\
\hline & $(6-12)$ & $(5-8)$ & \\
\hline \multirow[t]{2}{*}{ Blood hCG levels at diagnosis (mIU/mL) } & $17,551.3+$ & $37,876.1+$ & 0.18 \\
\hline & $20,483.0$ & $33,816.3$ & \\
\hline \multirow[t]{2}{*}{ Blood hCG levels at D\&C (mIU/mL) } & $15,360.8+$ & $17,840.5+$ & 0.78 \\
\hline & $20,344.9$ & $19,111.9$ & \\
\hline
\end{tabular}

There are no significant differences in characteristics between D\& C alone and combined treatment.

Table 3.

Comparison of background between cases with dilation and curettage (D\&C) alone and those with methotrexate + transcatheter arterial embolization + D\& $C$. 
A Minimally Invasive Hemostatic Strategy for Cesarean Scar Pregnancy and Cervical Pregnancy DOI: http://dx.doi.org/10.5772/intechopen.89666

\begin{tabular}{|c|c|c|c|}
\hline & $\begin{array}{c}\text { D\&C alone } \\
(\mathrm{n}: 6)\end{array}$ & MTX + TAE + D\&C (n:21) & $P$-value \\
\hline \multirow[t]{2}{*}{ Blood transfusion/>2500 mL blood loss } & $83.3 \%$ & $9.5 \%$ & 0.001 \\
\hline & $(5 / 6)$ & $(2 / 21)$ & \\
\hline \multirow[t]{2}{*}{ Wedge resection/hysterectomy } & $50.0 \%$ & $0 \%$ & 0.007 \\
\hline & $(3 / 6)$ & $(0 / 21)$ & \\
\hline Hospital stay (days) & $15.5+10.4$ & $9.6+8.4$ & 0.163 \\
\hline
\end{tabular}

Table 4 .

Comparison of outcome and additional treatment between D\&C alone and methotrexate + transcatheter arterial embolization + dilation and curettage $(D \& C)$.

cases with placenta previa on cesarean delivery scar, who were managed to leave the placenta in situ for placenta accreta spectrum disorders after cesarean section near term (median 36 weeks gestation; range 28-38 weeks gestation) in order to preserve the uterus without hysterectomy, the conservative therapy was successful in 25 (69.4\%) cases without any additional surgery [50]. Placental resorption occurred postpartum (median 89 days; range 6-510 days). Hysterectomy was performed for the other 11 cases, primarily owing to hemorrhage and/or infection. Considering smaller uterine blood supply and amount of gestational tissues in the first trimester than those in near term, conservative management after cessation of fetal heart beats in cesarean scar pregnancy may have higher success rate, if possible, with close postpartum follow-up for at least several months.

\section{Conclusion}

The basic policy for the management of non-tubal ectopic pregnancy, such as CSP and cervical pregnancy, is termination of pregnancy and preservation of the uterus, and the method of treatment should be selected according to the gestational week, presence/absence of fetal heart beats, the blood hCG level, and abundance of the blood supply adjacent to villi or the gestational sac. Recent reviews and reports support an interventional or a combination of surgical and medical approaches for treatment of unruptured CSP and cervical pregnancy rather than medical approach alone. In a national cohort study, surgical management appears to be associated with a high success rate, low complication rate, and short posttreatment follow-up.

Massive hemorrhage reportedly occurs in some cases with spontaneous rupture or after evacuation of the uterus without an accurate diagnosis. In cases experiencing massive hemorrhage, pressure hemostasis using balloon tamponade should first be performed. If such hemostasis proves to be ineffective, surgical excision or TAE should be selected next. If balloon tamponade or TAE is successful in controlling hemorrhage, conservative treatment is also a feasible option. In cases with coagulopathy, the blood fibrinogen level is often $<100 \mathrm{mg} / \mathrm{dL}$. Therefore, the treatment of coagulopathy should be performed by combined administration of concentrated coagulation factors (fibrinogen concentrate and cryoprecipitate) and fresh-frozen plasma promptly to obtain a blood fibrinogen level of at least $150-200 \mathrm{mg} / \mathrm{dL}$.

Recently, a minimally invasive hemostatic strategy in obstetrics, which aims to preserve uterine function and enhance the safety of subsequent pregnancies, has been developed. Therefore, we should reconsider uterus-preserving hemostatic 
strategies for critical hemorrhage and management of non-tubal ectopic pregnancy under these circumstances by using safe and minimally invasive treatment modalities. Moreover, if early detection of CSP is possible, more minimally invasive treatment might be another option of treatment such as double balloon conservative therapy or hysteroscopic resection without chemotherapy. Conservative therapy without chemotherapy and surgical therapy may be the other option. They were just followed up closely in outpatient clinic after cessation of fetal heart beats by local injection of $\mathrm{KCl}$.

The optimal treatment of CSP and cervical pregnancy is still unclear at present. Further evaluation of several therapies and hemostatic techniques by treating a large number of patients is necessary.

\section{Conflict of interest}

None.

\section{Author details}

Satoru Takeda ${ }^{1,2 *}$, Jun Takeda ${ }^{1}$, Takashi Yorifuji ${ }^{1}$ and Taro Koshiishi ${ }^{3}$

1 Department of Obstetrics and Gynecology, Faculty of Medicine, Juntendo University, Tokyo, Japan

2 Aiiku Research Institute for Maternal, Child Health and Welfare, Tokyo, Japan

3 Hagukumi Clinic for Mama and Kids, Kanagawa, Japan

*Address all correspondence to: stakeda@juntendo.ac.jp

\section{IntechOpen}

(C) 2019 The Author(s). Licensee IntechOpen. This chapter is distributed under the terms of the Creative Commons Attribution License (http://creativecommons.org/licenses/ by/3.0), which permits unrestricted use, distribution, and reproduction in any medium, provided the original work is properly cited. (cc) BY 


\section{References}

[1] Yorifuji T, Takeda J, Koshiishi T, Takeda S. Cesarean scar pregnancy. In: Takeda S, Hiramatsu Y, Konishi I, Sakuragi N, editors. OGS NOW, No. 10. Massive Obstetric Hemorrhage: Critical Care for Intractable Bleeding and Definite Strategies of Hemostasis. Tokyo: Medical View; 2012. pp. 64-71 in Japanese

[2] Takeda S, Takeda J, Makino S. A minimally invasive hemostatic strategy in obstetrics aiming to preserve uterine function and enhance the safety of subsequent pregnancies. Hypertens Res Pregnancy. 2019;7:9-15. DOI: 10.14390/ jsshp.HRP2018-013

[3] Sone M, Nakajima Y, Woodhams R, Shioyama Y, Tsurusaki M, Hiraki T, et al. Interventional radiology for critical hemorrhage in obstetrics: Japanese Society of Interventional Radiology (JSIR) procedural guidelines. Japanese Journal of Radiology. 2015;33(4):233-240. DOI: 10.1007/s11604-015-0399-0

[4] Inoue S, Masuyama H, Hiramatsu Y, Multi-Institutional Study Group of Transarterial Embolization for Massive Obstetric Haemorrhage in Chugoku \& Shikoku Area Society of Obstetrics and Gynecology. Efficacy of transarterial embolisation in the management of postpartum haemorrhage and its impact on subsequent pregnancies. The Australian \& New Zealand Journal of Obstetrics \& Gynaecology. 2014;54(6):541-545. DOI: 10.1111/ajo.12228

[5] Takeda J, Makino S, Ota A, Tawada T, Mitsuhashi N, Takeda S. Spontaneous uterine rupture at 32 weeks of gestation after previous uterine artery embolization. The Journal of Obstetrics and Gynaecology Research. 2014;40(1):243-246. DOI: 10.1111/ jog.12122

[6] Sano Y, Takeda J, Kuroda K, Makino S, Itakura A, Takeda S.
Embrittlement of uterus after uterine artery embolization: A case of uterine perforation. Hypertension Research in Pregnancy. 2016;4:42-44. DOI: 10.14390/jsshp.HRP2015-017

[7] Takeda J, Makino S, Takeda S. Hemostasis for massive hemorrhage during cesarean section. In: Schmolzer G, editor. Cesarean Delivery. London: IntechOpen; 2019 in press

[8] Chen HY et al. Observation of cesarean section scar by transvaginal ultrasonography. Ultrasound in Medicine \& Biology. 1990;16:443-447

[9] Larsen JV, Solomon MH. Pnegnancy in a uterine sca sacculus-an unusual cause of postabortal haemorrhage. A case report. South African Medical Journal = Suid-Afrikaanse Tydskrif vir Geneeskunde. 1978;53:142

[10] Seow KM, Huang LW, Lin YH, Lin MY, Tsai YL, Hwang JL. Cesarean scar pregnancy: Issues in management. Ultrasound in Obstetrics \& Gynecology. 2004;23:247-253. DOI: 10.1002/uog.974

[11] Harb HM, Knight M, Bottomley C, Overton C, Tobias A, Gallos ID, et al. Caesarean scar pregnancy in the UK: A national cohort study. BJOG: An International Journal of Obstetrics and Gynaecology. 2018;125(13):1663-1670. DOI: 10.1111/1471-0528.15255

[12] Vial Y, Petignat P, Hohlfeld P. Pregnancy in cesarean scar.

Ultrasound in Obstetrics \& Gynecology. 2000;16:592-593. DOI: 10.1046/j.1469-0705.2000.00300-2.x

[13] Jurkovic D, Hillaby K, Woelfer B, Lawrence A, Salim R, Elson CJ. First trimester diagnosis and management of pregnancies implanted into the lower uterine caesarean section scar. Ultrasound in Obstetrics \& Gynecology. 2003;21:220-227. DOI: 10.1002/uog.56 
[14] Rotas MA, Haberman S, Levgur M. Cesarean scar ectopic pregnancies: Ethiology, diagnosis and management. Obstetrics and Gynecology. 2006;107:1373-1377. DOI: 10.1097/01. AOG.0000218690.24494.ce

[15] Calì G, Timor-Tritsch IE, Palacios-Jaraquemada J, Monteaugudo A, Buca D, Forlani F, et al. Outcome of cesarean scar pregnancy managed expectantly: Systematic review and meta-analysis. Ultrasound in Obstetrics \& Gynecology. 2018;51(2):169-175. DOI: 10.1002/uog.17568

[16] Fylstra DL. Ectopic pregnancy within a cesarean scar: A review. Obstetrical \& Gynecological Survey. 2002;57(8):537-543. DOI: 10.1097/01. OGX.0000025517.33346.1E

[17] Timor-Tritsch IE, Monteagudo A, Santos R, Tsymbal T, Pineda G, Arslan AA. The diagnosis, treatment, and follow-up of cesarean scar pregnancy. American Journal of Obstetrics and Gynecology. 2012;207(1):44.e1-44.13. DOI: 10.1016/j. ajog.2012.04.018

[18] Timor-Tritsch IE, Monteagudo A. Unforeseen consequences of the increasing rate of cesarean deliveries: Early placenta accreta and cesarean scar pregnancy. A review. American Journal of Obstetrics and Gynecology. 2012;207(1):14-29. DOI: 10.1016/j. ajog.2012.03.007

[19] Jabeen K, Karuppaswamy J. Nonsurgical management of caesarean scar ectopic pregnancy-A five-year experience. Journal of Obstetrics and Gynaecology. 2018;38(8):1121-1127. DOI: $10.1080 / 01443615.2018 .1451986$

[20] Birch Petersen K, Hoffmann E, Rifbjerg Larsen C, Svarre Nielsen H. Cesarean scar pregnancy: A systematic review of treatment studies. Fertility and Sterility. 2016;105(4):958-967. DOI: 10.1016/j.fertnstert.2015.12.130
[21] Alalade AO, Smith FJE,

Kendall CE, Odejinmi F. Evidence-based management of non-tubal ectopic pregnancies. Journal of Obstetrics and Gynaecology. 2017;37(8):982-991. DOI: 10.1080/01443615.2017.1323852

[22] Maheux-Lacroix S, Li F, Bujold E, Nesbitt-Hawes E, Deans R, Abbott J. Cesarean scar pregnancies: A systematic review of treatment options. Journal of Minimally Invasive Gynecology. 2017;24(6):915-925. DOI: 10.1016/j.jmig.2017.05.019

[23] Kim SY, Yoon SR, Kim MJ, Chung JH, Kim MY, Lee SW. Cesarean scar pregnancy; diagnosis and management between 2003 and 2015 in a single center. Taiwanese Journal of Obstetrics \& Gynecology. 2018;57(5):688-691. DOI: 10.1016/j. tjog.2018.08.013

[24] Giampaolino P, De Rosa N, Morra I, Bertrando A, Di Spiezio SA, Zizolfi B, et al. Management of cesarean scar pregnancy: A single-institution retrospective review. BioMed Research International. 2018;2018:6486407. DOI: 10.1155/2018/6486407. eCollection 2018

[25] Gonzalez N, Tulandi T. Cesarean scar pregnancy: A systematic review. Journal of Minimally Invasive Gynecology. 2017;24(5):731-738. DOI: 10.1016/j.jmig.2017.02.020

[26] Wang DB, Chen YH, Zhang ZF, Chen P, Liu KR, Li Y, et al. Evaluation of the transvaginal resection of low-segment cesarean scar ectopic pregnancies. Fertility and Sterility. 2014;101(2):602-606. DOI: 10.1016/j. fertnstert.2013.10.024

[27] Peng M, Li L, Ding Y, Yu L, Deng Y, Zheng J, et al. Exploration of the successful treatment algorithms used in 23 cases of early live cesarean scar pregnancy. Gynecologic and Obstetric Investigation. 2015;79(2):139-144. DOI: $10.1159 / 000368400$ 
[28] Jurkovic D, Knez J, Appiah A, Farahani L, Mavrelos D, Ross JA. Surgical treatment of cesarean scar ectopic pregnancy: Efficacy and safety of ultrasound-guided suction curettage. Ultrasound in Obstetrics \& Gynecology. 2016;47(4):511-517. DOI: 10.1002/ uog.15857

[29] Ades A, Parghi S. Laparoscopic resection of cesarean scar ectopic pregnancy. Journal of Minimally Invasive Gynecology. 2017;24(4):533-535. DOI: 10.1016/j.jmig.2016.11.006

[30] Kwon YS, Cho YM, Im KS, Yoo SB, Hyung SW. Transient occlusion of uterine arteries in procedures with high risk of uterine bleeding. Journal of the Society of Laparoendoscopic Surgeons. 2019;23(1):pii: e2018.00072. DOI: 10.4293/JSLS.2018.00072

[31] Jurkovic D, Ben-Nagi J, Ofilli-Yebovi D, Sawyer E, Helmy S, Yazbek J. Efficacy of Shirodkar cervical suture in securing hemostasis following surgical evacuation of cesarean scar ectopic pregnancy. Ultrasound in Obstetrics \& Gynecology. 2007;30(1):95-100. DOI: 10.1002/ uog.4058

[32] Heikinheimo O, Leminen A, Cacciatore B, Rutanen EM, Kajanoja P. Advanced cervical pregnancy: Uterussparing therapy initiated with a combination of methotrexate and mifepristone followed by evacuation and local hemostatic measures. Acta Obstetricia et Gynecologica Scandinavica. 2004;83(2):211-213

[33] Bakour SH, Thompson PK, Khan KS. Successful conservative management of cervical ectopic pregnancy with combination of methotrexate, mifepristone, surgical evacuation and tamponade using a double balloon three-way catheter. Journal of Obstetrics and Gynaecology. 2005;25(6):616-618. DOI: $10.1080 / 01443610500243620$
[34] Gómez García MT, Aguarón Benitez G, Barberá Belda B, Callejón Rodríguez C, González Merlo G. Medical therapy (methotrexate and mifepristone) alone or in combination with another type of therapy for the management of cervical or interstitial ectopic pregnancy. European Journal of Obstetrics, Gynecology, and Reproductive Biology. 2012;165(1):77-81. DOI: 10.1016/j.ejogrb.2012.06.024

[35] Di Spiezio Sardo A, Vieira MDC, Laganà AS, Chiofalo B, Vitale SG, Scala M, et al. Combined systemic and hysteroscopic intra-amniotic injection of methotrexate associated with hysteroscopic resection for cervical pregnancy: A cutting-edge approach for an uncommon condition. The Eurasian Journal of Medicine. 2017;49(1):66-68. DOI: 10.5152/eurasianjmed.2017.16215

[36] Trambert JJ, Einstein MH, Banks E, Frost A, Goldberg GL. Uterine artery embolization in the management of vaginal bleeding from cervical pregnancy: A case series. The Journal of Reproductive Medicine. 2005;50(11):844-850

[37] Martinelli P, Maruotti GM, Oppedisano R, Agangi A, Mazzarelli LL, Votino $\mathrm{C}$, et al. Is uterine artery embolization for cervical ectopic pregnancy always safe? Journal of Minimally Invasive Gynecology. 2007;14(6):758-763. DOI: 10.1016/j. jmig.2007.05.017

[38] Ben Farhat L, Ben Salah Y, Askri A, Dali N, Hendaoui L. Conservative treatment of a cervical twin pregnancy with uterine artery embolization. Diagnostic and Interventional Radiology. 2010;16(3):248-250. DOI: 10.4261/1305-3825.DIR.2073-08.1

[39] Zhou Q, Young D, Vingan H. Uterine artery embolization for cervical ectopic pregnancy. Radiology Case Reports. 2015;10(4):72-75. DOI: 10.1016/j. radcr.2015.08.004. eCollection 2015 Dec 
[40] Mashiach S, Admon D, Oelsner G, Paz B, Achiron R, Zalel Y. Cervical shirodkar cerclage may be the treatment modality of choice for cervical pregnancy. Human Reproduction. 2002;17(2):493-496. DOI: 10.1093/ humrep/17.2.493

[41] Mancuso A, Carlo Stella N, Dugo C, de Vivo A, Priola V. A simple surgical approach in the treatment of cervical pregnancy. Journal of Obstetrics and Gynaecology. 2007;27(2):191-192. DOI: 10.1080/01443610601137747

[42] Faschingbauer F, Mueller A, Voigt F, Beckmann MW, Goecke TW. Treatment of heterotopic cervical pregnancies. Fertility and Sterility. 2011;95(5):1787. e9-1787.13. DOI: 10.1016/j.fertnstert. 2010.10.043

[43] Moragianni VA, Hamar BD, McArdle C, Ryley DA. Management of a cervical heterotopic pregnancy presenting with first-trimester bleeding: Case report and review of the literature. Fertility and Sterility. 2012;98(1):89-94. DOI: 10.1016/j.fertnstert.2012.04.003

[44] Mangino FP, Romano F, Di Lorenzo G, Buonomo F, De Santo D, Scrimin F, et al. Total hysteroscopic treatment of cervical pregnancy: The 2-step technique. Journal of Minimally Invasive Gynecology. 2019;pii:S15534650(19)30053-6. DOI: 10.1016/j. jmig.2019.01.009

[45] Timor-Tritsch IE, Monteagudo A, Bennett T-A, Foley C. A new minimally invasive treatment for cesarean scar pregnancy and cervical pregnancy. American Journal of Obstetrics and Gynecology. 2016;351:e1-e8. DOI: 10.1016/j.ajog.2016.03.010

[46] Yorifuji T, Takeda J, Makino S, Tanaka T, Itakura A, Takeda S. Evaluation of the effectiveness of metreurynters for balloon tamponade. Hypertension Research in Pregnancy.
2018;6:26-29. DOI: doi.org/10.14390/ jsshp.HRP2018-001

[47] Makino S, Takeda J, Hirai C, Itakura A, Takeda S. Uterine balloon tamponade as a test to assess further treatment. Acta Obstetricia et Gynecologica Scandinavica.

2015;94(5):556. DOI: 10.1111/aogs.12599

[48] Takeda J, Takeda S. Management of disseminated intravascular coagulation associated with placental abruption and measures to improve outcomes. Obstetrics \& Gynecology Science. 2019;62:e52. DOI: 10.5468/ ogs.2019.62.e52

[49] Makino S, Hirai C, Itakura A, Takeda S, Yoshikawa H, Li Z, et al. Evaluation of uterine blood flow: A new method using contrast-enhanced ultrasound. European Journal of Obstetrics, Gynecology, and Reproductive Biology. 2015;191:143-144. DOI: 10.1016/j.ejogrb.2015.04.009

[50] Miyakoshi K, Otani T, Kondoh E, Makino S, Tanaka M, Takeda S. Perinatal research network in Japan.

Retrospective multicenter study of leaving the placenta in situ for patients with placenta previa on a cesarean scar. International Journal of Gynaecology and Obstetrics. 2018;140(3):345-351. DOI: $10.1002 /$ ijgo.12397 


\title{
Broad Ligament Pregnancy
}

\author{
Stelios Fiorentzis, Theodoros Margetousakis, \\ Chrysostomos Georgellis, Pantelis Kotridis, \\ Dimitra Oikonomopoulou, Alexandros Karamperis, \\ Georgia Karamperi-Sotiropoulou and Dionysios Karavyrakis
}

\begin{abstract}
An ectopic pregnancy implanted between the leaves of the broad ligament is a challenge to diagnose and manage. It can be found in almost any week of pregnancy, and it can present with a great spectrum of symptoms. This makes it necessary for the clinician to keep a high level of alertness and suspicion for this uncommon diagnosis and to be prepared when treating any ectopic pregnancy with poor response to medical treatment. The broad ligament surrounds a number of anatomical structures, structures necessary to be preserved when removing the ectopic pregnancy. This can be a challenge for the surgeon, especially when the patient presents with severe symptoms and the pregnancy is advanced. In these cases, even hysterectomies have been reported, a severe operation especially when the patient is young and without having completed her family planning. Finally, in the last three decades with the increasing widespread of laparoscopy and the added experience, there is a growing effort to manage these ectopic pregnancies with minimally invasive procedures, offering the patient a faster recovery, minimum blood loss, and lower surgical morbidity.
\end{abstract}

Keywords: broad ligament, ectopic pregnancy, abdominal pain, laparotomy, laparoscopy, hysterectomy

\section{Introduction}

One of the rarest places of an ectopic pregnancy to be implanted is between the leaves of the broad ligament. Loschge in 1816 made the first known report of a broad ligament pregnancy [1], while Champion et al. in 1938 published a large series of 62 cases [2]. Kennedy in 1925, after recording one broad ligament pregnancy among 613 ectopic cases, calculated the incidence of intraligamentary pregnancy as 1 for every 183,900 pregnancies [3]. Even today when the medical literature is so extended and continuing, the exact incidence of this rare type of ectopic pregnancy is difficult to be exactly calculated. The extensive use of assisted reproductive technology has made even more difficult tracking every case. The rarity of ectopic pregnancies in the broad ligament makes it challenging for the clinician to diagnose, and it needs a high index of suspicion. In most of cases the diagnosis is done during an operation for treatment of a presumed ruptured tubal pregnancy. Despite the fact that most cases are diagnosed in the first trimester of pregnancy, there are a few cases reported that the diagnosis was not made until the third trimester and the final outcome was a 
live birth. The rarity of these cases also makes it impossible to find extensive series of patients so as to determine the best treatment, making the clinician rely on previous case reports and the methods used in other treated cases.

\section{Anatomy}

The broad ligament of the uterus is a double-layer fold of peritoneum (anterior and posterior leaves) extending from each side of the uterus to the lateral pelvic walls and the pelvic floor. It covers the lateral uterine corpus and the upper cervix as well. The structures within the broad ligament (uterine tubes, ovarian artery, uterine artery, ovarian ligament, round ligament of the uterus, suspensory ligament of the ovary, ovary) are considered retroperitoneal. The broad ligament itself is composed of visceral and parietal peritonea that contain smooth muscle and connective tissue $[4,5]$.

\section{Pathophysiology}

The way the trophoblast can be implanted retroperitoneally is still not clear. Broad ligament pregnancies are classified as abdominal ectopic pregnancies and can be further classified as primary or secondary. Many of them are considered to have started as a tubal or ovarian pregnancy, which later ruptured intraperitoneally and was implanted again in another location [6]. Studdiford in 1942 [7] suggested three criteria, later modified by Friedrich and Rankin [8], for the diagnosis of a primary peritoneal pregnancy, (i) normal tube and ovaries with no evidence of recent or remote surgery, (ii) absence of any uteroperitoneal fistula, and (iii) the presence of a pregnancy related exclusively to the peritoneal surface and young enough to eliminate the possibility of secondary implantation following a primary nidation in the tube.

Sotus in 1977, in a case report of a retroperitoneal ectopic pregnancy, hypothesized that an ectopic trophoblast could invade and penetrate the peritoneum, which soon thereafter covered the gestational tissues [9].

Another possible way for retroperitoneal implantation is during the embryo transfer in IVF, with uterine perforation and direct retroperitoneal placement. The arguments against this proposal are based on the softness and the flexibility of the catheters used for embryo transfer [7]. Another argument against this mechanism that has been reported is a broad ligament pregnancy after an ultrasound-guided embryo transfer [11].

The above proposals cannot explain how a broad ligament pregnancy can occur in a patient with bilateral salpingectomy. The possibility of a microscopic fistulous tract through which the transferred embryos entered the abdominal cavity has also been proposed by Fisch et al. in 1996 [10]. Apantaku et al., also in 1996, proposed the possibility of recanalization of a fallopian tube stump in a patient with bilateral salpingectomy [11].

Fisch et al. made another proposal trying to explain a reported broad ligament pregnancy. They suggested that the spermatozoa could enter the abdominal cavity through a cornual fistulous tract and fertilize an oocyte already migrated there. This proposal was made when trying to explain a broad ligament pregnancy to a patient with bilateral salpingectomy who had intercourse the day after follicular aspiration [10].

Finally it has to be mentioned that Deshpande et al. in 1999 reported a twin pregnancy in the broad ligament to a patient who underwent in vitro fertilization [12], while in 2001, Phupong et al. reported a twin pregnancy in the broad ligament after spontaneous conception [13]. 


\section{Symptoms}

There is a wide range of symptoms which can lead a patient to seek help and find out that she is pregnant with an ectopic pregnancy. Most of the symptoms are the same as for every other ectopic pregnancy, with abdominal pain and vaginal bleeding to be the most prominent. The abdominal pain can be severe and of sudden onset [14] or intermittent and associated with other symptoms [15]. The pain can also be characterized as "mild" or "moderate" $[16,17]$ or just a "discomfort" [18], or finally it can be gradually increased in intensity [19]. The location of the pain has also to be reported to be related with the side of the ectopic pregnancy [19-21]. The duration of the pain can also vary between different cases, with even an extreme case of 4 months of intermittent pain to have been reported [22]. Finally it is not unusual for a broad ligament pregnancy to be completely asymptomatic and to be found during an early routine visit $[11,12]$.

The vaginal bleeding, if present, can also be of different severities. Cases with just vaginal spotting $[21,23,24]$ have been reported, while in other reported cases, vaginal bleeding was present and more severe [22, 25-31], leading the patient to seek medical assistance.

Other symptoms that have been reported in an early broad ligament pregnancy include nausea [20, 24, 32], chills and fever [20], vomiting [20, 28], and dysuria [15]. These symptoms can be present alone or accompanying others, with various severities and to be evaluated as important or of no importance by the patient.

Despite the modern techniques and progress, there are places (more often in the developing world) where a pregnant woman cannot have access to proper antenatal care, making possible for an intraligamentary pregnancy to advance. In such a case, it is possible for the pregnant woman to seek help because of inability to perceive fetal movement $[33,34]$ or to be referred to a more specialized hospital due to severe oligamnion [35, 36] or intrauterine growth retardation [35]. Abdominal pain and vaginal bleeding are other possible symptoms for an advanced intraligamentary pregnancy like any other ectopic pregnancy.

It is also possible for a broad ligament pregnancy to have no symptom at all and to be found during a routine visit. Suchánková et al. reported a case where a patient with bilateral salpingectomy was referred to hospital from an assisted reproduction center to solve suspicion of molar intrauterine pregnancy, while the ultrasound and the following laparoscopy revealed a right broad ligament pregnancy [37]. Apantaku et al. in 2006 presented a case where a broad ligament pregnancy was discovered during a routine visit of a patient who had an IVF 6 weeks earlier [11], while Deshpande et al. in 1999 presented the first case of a twin broad ligament pregnancy 7 weeks after IVF [12]. Siow et al. in 2004 reported a case of a 10-week asymptomatic woman who presented for routine antenatal care and was found with a right adnexal mass and empty uterus. This was also the first published broad ligament pregnancy which was managed laparoscopically [38]. Laparoscopy was also used to treat another case presented by Cosentino et al. in 2017 of an asymptomatic woman who presented to be submitted to a noninvasive prenatal diagnosis procedure and was found to have a broad ligament pregnancy [39].

Finally there have been reported cases where the pregnancy went uneventfully until term. Seckin et al. in 2011 reported a case with a primigravid patient who was admitted due to breech presentation and oligamnion. Because of the breech presentation, they decided to proceed with primary cesarean section, where an unruptured gestational sac was found between the layers of the broad ligament with both adnexa being normal [36]. In another case report by Schramm in 1982, a case of a patient who presented with pains every 4-5 minutes for 1 hour was described. Another three mild contractions the next 90 minutes were recorded, and after that 
it was difficult to identify the fetal lie and a definite uterine outline. Emergency laparotomy was carried out, and the placenta was found to be attached to the anterior layer of the broad ligament [40]. The above cases were with favorable result; both mother and child survived through gestation and birth. Rakotomahenina et al. in 2014 reported a post-term pregnancy in the right broad ligament. The patient was not followed up during gestation and presented to the hospital at the 44th week. The fetus was dead in transversal position and was extracted from the right broad ligament by laparotomy [41]. This case showed that a broad ligament pregnancy can reach term without any serious symptoms.

From the above it can be concluded that a broad ligament pregnancy can present with a variety of symptoms or no symptom at all. When preparing for an operative solution for an ectopic pregnancy, the clinician must be prepared for this rare presentation, and it needs a high level of suspicion. When conservative management with methotrexate is chosen for an ectopic pregnancy and does not have the expected results, broad ligament pregnancy should also be part of the differential diagnosis.

\section{Diagnosis}

The diagnosis of an ectopic pregnancy located in the broad ligament is difficult to be established preoperatively. In most cases the broad ligament diagnosis is done during the operation for a misdiagnosed tubal pregnancy, which can be either by laparotomy or laparoscopy. In this case the pregnancy can be found laterally to the uterus, medial to the pelvic side walls, superior to the pelvic floor, and inferior to the fallopian tube [42].

Ultrasound, transvaginal or abdominal, is the method of choice to identify the location of an ectopic pregnancy. If finding the site of an ectopic pregnancy is difficult, the easiest way to rule out an ectopic pregnancy is to identify an intrauterine one [43], something almost $100 \%$ possible in a gestation greater than 5,5 weeks [44, 45]. An empty uterus with positive pregnancy test and the presence of a gestational sac or mass outside the uterus gives high suspicion for ectopic pregnancy [46]. A broad ligament pregnancy can grow significantly before giving any symptoms [47], in which the size can lead to distorted anatomy and increased difficulty in making an accurate diagnosis [48]. Phupong et al. in 2003 based on an empty uterus, a mass with a single viable fetus in a gestational sac located just beside the right side of the lower part of the uterus, and the clinical findings and their experience in a previous managed case, they reported a correct preoperative diagnosis by transvaginal ultrasound [31]. These two findings (empty uterus and an ectopic pregnancy mass just beside the lower part of the uterus when using transvaginal ultrasound) are two suggested ultrasonographic clues which can make the clinician suspect a broad ligament pregnancy [31]. Also in 2001 Sharma et al. reported that they managed to diagnose preoperatively a broad ligament pregnancy based on the anterior place with peritoneal reflection of the placenta combined with free fluid in the abdominal cavity and a bulky with thick endometrium uterus [28]. Allibone et al. in 1981 [49] suggested six criteria in order to assist the identification of an abdominal pregnancy: (a) demonstration of a fetus in a gestational sac outside the uterus, or the depiction of an abdominal or pelvic mass, identifiable as the uterus, separate from the fetus $[49,50]$, (b) failure to see a uterine wall between the fetus or products of conception and the urinary bladder, (c) recognition of a close approximation of fetal parts to the maternal abdominal wall, or gestational products like the placenta, both features alerting the sonographer to the absence of enveloping uterine walls, (d) demonstration of eccentric position and/or abnormal attitude of the fetus, (e) 
localization of the placenta outside the confines of the uterine cavity, (f) visualization of the placenta immediately adjacent to the fetal chest and head with no intervening amniotic fluid [51].

Magnetic resonance imaging (MRI) can offer great help to the diagnosis of an ectopic pregnancy, especially when then location is unknown. MRI can also offer valuable information for the preoperative planning so as to avoid cutting in the placenta and reduce the bleeding during surgery $[52,53]$. MRI can also highlight the involvement of pelvic structures and organs in an ectopic pregnancy, providing more helpful data to the preoperative planning [46]. Finally, it is also important to remember the contraindication of some contrast agents in pregnancy, which has to be considered when the life of the mother is at risk. In any case an informed consent should be obtained by the patient [54].

Angiogram is another tool which can prove its usefulness preoperatively, in order to reveal the location of the placental vessels, while embolization and pre- or postoperative can be used to control hemorrhage. Embolization could also be used for difficult-to-reach vessels intraoperatively $[49,55]$, although the location of a broad ligament pregnancy and the fact that such a pregnancy as often as not presents with acute abdomen limit the possible use of the above techniques.

\section{Management}

The rarity of this type of ectopic pregnancy makes it impossible to have prospective trials so as to conclude the best possible treatment. The reported cases that already can be found in the international medical literature can propose different management options [56], taking into consideration the different means every managing clinician can use.

Broad ligament pregnancies are difficult to be diagnosed before the time of surgical management. Therefore, methotrexate cannot be considered the first-line treatment for these conditions but may have been initiated if diagnosed incorrectly as tubal pregnancies [22, 57]. Despite that, Direkvand-Moghadam et al. in 2015 reported a case where they treated a left broad ligament pregnancy with a single dose of methotrexate in a patient with left abdominal pain, 33 days since last menstrual period, $\beta$-hcg = 212 IU.L, and no abnormal sonographic findings. The maximum level of $\beta$-hcg was 659 IU.L, the forty sixth day after the last menstrual period, and returned to normal 3 weeks after the injection [21].

The way a patient with broad ligament pregnancy presents (abdominal pain in the first trimester) makes it very difficult to follow conservative treatment in order to achieve a favorable result such a live birth. Cachón López et al. in 1989 reported such a case of an 18-year-old woman diagnosed with a 30.4 week abdominal pregnancy. The diagnosis was established by clinical and imaging tests. They decided to keep the patient under close surveillance for 2 weeks and to induce fetal lung maturation. At 32.4 weeks of gestation, a laparotomy was performed giving birth to a $1.100 \mathrm{gr}$ baby. The placenta was found on the surface of the anterior leaf of the broad ligament and was removed together with the right salpinx [58].

Laparoscopic management of a broad ligament pregnancy is not impossible, and it has many advantages as shown by the laparoscopic management of abdominal pregnancies in other unusual sites. These advantages include the faster recovery of the patient, the lower surgical morbidity, and the better control of the blood loss [38]. It can be considered when the size is small $[23,59]$ and the patient stable. The presence of proficient laparoscopists and the option to convert to laparotomy at any time are also crucial [38]. Olsen in 1997 reported the first case of a successful laparoscopic management of a broad ligament pregnancy [23], while in 2004 Siow 
et al. reported a successful laparoscopic management of a pregnancy presenting with a $6.5 \mathrm{~cm}$ mass. Siow et al. injected vasopressin into the broad ligament before starting the excision, the pregnancy was removed, and the surgery was completed uncomplicated [38]. Since then there has been an increase in the reports of broad ligament pregnancies which were managed with laparoscopy $[11,17,24,25,30$, 60-62], while Mo et al. in 2018, in the case they reported, managed laparoscopically a broad ligament pregnancy with a hemoperitoneum of about $2800 \mathrm{ml}$. In most cases coagulation was used to control the breeding. Cheung et al. in 2014 reported that they needed to use three 1-0 Biosyn stitches in order to close the implantation site inside the broad ligament, so as to control the bleeding [17], while Kar in 2012 used superficial infiltration of Pitressin (vasopressin) so as to control the hemorrhage from the base [62]. Finally in a case report by Yang et al. in 2017, they used absorbable hemostatic cellulose which was inserted into the retroperitoneal space in order to prevent bleeding [63].

Despite the extensive use of laparoscopy and the willingness to treat an ectopic pregnancy with more conservative ways, explorative laparotomy is still the way most cases are treated. The presentation to the hospital-most common with abdominal pain — of a missed broad ligament pregnancy plays an important role to this. Laparotomies have been reported in order to treat a wide spectrum of broad ligament pregnancies, starting with 5 weeks of amenorrhea [60], till broad ligament pregnancies at term $[15,36]$ which were admitted for an emergency cesarian section. Finally a case of a post-term broad ligament pregnancy has been reported [41].

In the published medical literature, different outcomes for women who underwent laparotomy or laparoscopy for a broad ligament pregnancy can also be found. There have been reported cases where the size of the pregnancy made it possible to be removed without any damage to the surrounding organs $[10-12,14,15,17,19-26$, $33,36-38,41,60-62,64-66]$, where in other cases salpingectomy [18, 27, 31, 32, 40, $56,58,67]$, oophorectomy [30], or salpingo-oophorectomy [28, 34, 35, 39] was necessary to be performed due to the damage these organs presented. There have also been reported cases where the need for hysterectomy presented [20, 29, 40, 68], and finally in a report by Wolfe and Neigus in 1953, among three cases reported, the two patients died [20]. The above show that a broad ligament pregnancy can be a life-threatening situation or a cause of a serious disability, especially when excision of ovaries or the uterus is a necessity in order to save the patient. It also makes it necessary to inform the patient before the operation of the possible outcomes, especially when the suspicion of a broad ligament pregnancy is present.

Finally, the fact that a missed broad ligament pregnancy can present from an early week of amenorrhea or it can reach term and post-term, with a great range of symptoms (from none to very acute) makes the broad ligament pregnancy part of the differential diagnosis of nearly every pregnant woman presenting to the emergency room, no matter in which week of pregnancy she is. In the case that a broad ligament pregnancy is considered to be a possibility, it is important for the managing surgeon to gather the best available team. Because of the complexity of these cases, this team cannot be limited to gynecologists but should also include-if possible-general surgeons, urologists, and interventional radiologists. Each one of them can play an important role to the management of a broad ligament pregnancy depending on the size of the pregnancy and the damage to the surrounding organs.

\section{Conclusions}

An ectopic pregnancy located between the leaves of the broad ligament is a rare but possibly life-threatening presentation of an ectopic pregnancy. The fact that it 
can be easily missed is it can cause worrying symptoms in any week of pregnancy, or it can go uneventful till term makes it a possibility in differential diagnosis of almost every pregnant woman presenting to the emergencies. The diagnosis preoperatively is also difficult to be established. MRI can play an important role if there is ultrasound suspicion for a broad ligament pregnancy. It must also be mentioned that, although there is growing need to manage ectopic pregnancies with minimal invasive techniques, methotrexate cannot be considered a first-line treatment for this type of ectopic pregnancy. On the other hand, there are numerous reports, and becoming more often every year, of broad ligament pregnancies managed laparoscopically. Finally, every gynecologist or surgeon who must operate a woman with a suspicion of a broad ligament pregnancy must provide the patient the best information for possible excision of organs crucial to reproduction.

\section{Author details}

Stelios Fiorentzis ${ }^{1}$, Theodoros Margetousakis ${ }^{2}$, Chrysostomos Georgellis ${ }^{3}$, Pantelis Kotridis ${ }^{4}$, Dimitra Oikonomopoulou ${ }^{5}$, Alexandros Karamperis ${ }^{6}$, Georgia Karamperi-Sotiropoulou ${ }^{7}$ and Dionysios Karavyrakis ${ }^{1 *}$

1 Department of Obstetrics and Gynecology, General Hospital of Sitia, Greece

2 Department of General Surgery, General Hospital of Sitia, Greece

3 Department of Urology, University Hospital of Alexandroupolis, Greece

4 Department of Obstetrics and Gynecology, General Hospital of Kalymnos, Greece

5 Department of Obstetrics and Gynecology, Alexandra Hospital, Athens, Greece

6 Department of Obstetrics and Gynecology, Elefsina General Hospital, Greece

7 Health Center Kalyvion, Saronikos, Greece

*Address all correspondence to: dkaravyrakis@gmail.com

\section{IntechOpen}

(C) 2019 The Author(s). Licensee IntechOpen. This chapter is distributed under the terms of the Creative Commons Attribution License (http://creativecommons.org/licenses/ by/3.0), which permits unrestricted use, distribution, and reproduction in any medium, provided the original work is properly cited. (cc) BY 


\section{References}

[1] Loschge. Beschreibung einer merkwuerdigen schwangerschaft innerhalb des breiten mutterbandes. Arch Fuer Medizinische Erfahrung. 1818;2:218

[2] Champion PK, Tessitore NJ. Intraligamentary pregnancy. American Journal of Obstetrics and Gynecology. 1938;36(2):281-293. Available from: https://linkinghub.elsevier.com/ retrieve/pii/S0002937838910002

[3] Kennedy WT. Intraligamentous pregnancy. American Journal of Obstetrics and Gynecology. 1925;10(6):858-862. Available from: https://linkinghub.elsevier.com/ retrieve/pii/S0002937825904632

[4] Bajowa Edozien GY. Sexual offenses, adult: Normal anogenital anatomy and variants. In: Encyclopedia of Forensic and Legal Medicine [Internet]. 2nd ed. Cambridge, Massachusetts: Elsevier; 2016. pp. 286-311. Available from: https://linkinghub.elsevier.com/ retrieve/pii/B9780128000342000744

[5] Chiva LM, Magrina J. Abdominal and pelvic anatomy. In: Principles of Gynecologic Oncology Surgery [Internet]. Philadelphia: Elsevier; 2018. pp. 3-49. Available from: https:// linkinghub.elsevier.com/retrieve/pii/ B978032342878100002X

[6] Ouassour S, Filali AA, Raiss M, Bezad R, Tazi Z, Alami MH, et al. Retroperitoneal ectopic pregnancy: Diagnosis and therapeutic challenges. Case Reports in Surgery. 2017;2017:9871865. Available from: http://www.ncbi.nlm.nih.gov/ pubmed/29201485

[7] Studdiford WE. Primary peritoneal pregnancy. American Journal of Obstetrics and Gynecology. 1942;44(3):487-491. Available from: https://linkinghub.elsevier.com/ retrieve/pii/S0002937842904885
[8] Friedrich EG, Rankin CA. Primary pelvic peritoneal pregnancy. Obstetrics and Gynecology. 1968;31(5):649-653. Available from: http://www.ncbi.nlm. nih.gov/pubmed/5646396

[9] Sotus PC. Retroperitoneal ectopic pregnancy: A case report. JAMA. 1977;238(13):1363-1364. Available from: http://www.ncbi.nlm.nih.gov/ pubmed/578188

[10] Fisch B, Peled Y, Kaplan B, Zehavi S, Neri A. Abdominal pregnancy following in vitro fertilization in a patient with previous bilateral salpingectomy. Obstetrics and Gynecology. 1996;88(4 Pt 2):642-643. Available from: http://www.ncbi.nlm.nih.gov/ pubmed/8841236

[11] Apantaku O, Rana P, Inglis T. Broad ligament ectopic pregnancy following in-vitro fertilisation in a patient with previous bilateral salpingectomy. Journal of Obstetrics and Gynaecology. 2006;26(5):474. Available from: http://www.ncbi.nlm.nih.gov/ pubmed/16846888

[12] Deshpande N, Mathers A, Acharya U. Broad ligament twin pregnancy following in-vitro fertilization. Human Reproduction. 1999;14, 3:852-854. Available from: http://www.ncbi.nlm.nih.gov/ pubmed/10221727

[13] Phupong V, Tekasakul P, Kankaew K. Broad ligament twin pregnancy. A case report. The Journal of Reproductive Medicine. 2001;46(2):144-146. Available from: http://www.ncbi.nlm.nih.gov/ pubmed/11255815

[14] Hatada Y. The pedunculated type of primary peritoneal pregnancy implanted on the infundibulopelvic ligament. Obstetrics and Gynecology. 
1993;82(4 Pt 2 Suppl):693-695. Available from: http://www.ncbi.nlm.nih.gov/ pubmed/8378016

[15] Dahab AA, Aburass R, Shawkat W, Babgi R, Essa O, Mujallid RH. Full-term extrauterine abdominal pregnancy: A case report. Journal of Medical Case Reports. 2011;5:531. Available from: http://www.ncbi.nlm.nih.gov/ pubmed/22040324

[16] Badr S, Ghareep A-N, Abdulla LM, Hassanein R. Ectopic pregnancy in uncommon implantation sites. Egyptian Journal of Radiology and Nuclear Medicine. 2013;44(1):121130 Available from: https:// linkinghub.elsevier.com/retrieve/pii/ S0378603X1200099X

[17] Cheung CS, Cheung VYT. Broad Ligament Ectopic Pregnancy. CRSLS MIS Case Reports from SLS [Internet]. 2014;18(4):1-5. Available from: http:// crsls.sls.org/2014-00102

[18] Khare V, Shrivastava S, Mishra K. An unusual presentation of live fetus in broad ligament: A case report. Journal of Evolution of Medical and Dental Sciences. 2014;3(01):223-225. Available from: http://www.jemds.com/data_pdf/ Drveena khare.pdf

[19] Audifred Salomón JR, Herrera Ortiz A, González Medrano MG, Estrada Rivera SF. Ectopic intraligamentary pregnancy. Ginecología y Obstetricia de México. 2013;81(4):211-214. Available from: http://www.ncbi.nlm.nih.gov/ pubmed/23720935

[20] Wolfe SA, Neigus I. Broad-ligament pregnancy with report of three early cases. American Journal of Obstetrics and Gynecology. 1953;66(1):106-117. Available from: http://www.ncbi.nlm. nih.gov/pubmed/13057986

[21] Direkvand-Moghadam A, Direkvand-Moghadam A. Broad ligament ectopic pregnancy: A case report. Der Pharmacia Lettre. 2015;7(10):1-4

[22] Zu M, Zhao GQ, Liu ZQ, Zhang HT, Chen L, Zhao DH. A case report of a patient with high $\beta$-hCG levels after operation because of primary broad ligament pregnancy. Clinical and Experimental Obstetrics \& Gynecology. 2017;44(1):138-142. Available from: http://www.ncbi.nlm.nih.gov/ pubmed/29714884

[23] Olsen ME. Laparoscopic treatment of intraligamentous pregnancy. Obstetrics and Gynecology. 1997;89(5 Pt 2):862. Available from: http://www. ncbi.nlm.nih.gov/pubmed/9166356

[24] Nayar J, Nair SS. Broad ligament pregnancy-Success story of a laparoscopically managed case. Journal of Clinical and Diagnostic Research. 2016;10(7):QD04-QD05. Available from: http://www.ncbi.nlm.nih.gov/ pubmed/27630914

[25] Cormio G, Ceci O, Loverro G, Bettocchi S. Spontaneous left broad ligament pregnancy after ipsilateral salpingo-oophorectomy. Journal of Minimally Invasive Gynecology. 2006;13(2):84-85. Available from: http://www.ncbi.nlm.nih.gov/ pubmed/16527706

[26] Kutlesic R, Lukic B, Kutlesic M, Popovic J, Stefanovic M, Vukomanovic P, et al. Unruptured retroperitoneal pregnancy implanted in the left broad ligament: A case report. Vojnosanitetski Pregled. 2017;74(2):177-183. Available from: http://www.doiserbia.nb.rs/ Article.aspx?ID=0042-84501600160K

[27] Hameed J, Radhika, Haseena, Lakshmi S, Jaisree, Ahamed N. A case of broad ligament pregnancy. International Journal of Scientific Study. 2014;2(4):77-79

[28] Sharma S, Pathak N, Goraya S, Mohan P. Broad ligament ectopic 
pregnancy.SriLankaJournalofObstetrics and Gynaecology. 2012;33(2):60. Available from: https://sljog.sljol.info/ article/10.4038/sljog.v33i2.4008/

[29] Atalla RK, Murphy PC, Balachandar C. Combined intrauterine and broad ligament ectopic pregnancy. Journal of Obstetrics and Gynaecology. 1997;17(2):203. Available from: http://www.ncbi.nlm.nih.gov/ pubmed/15511824

[30] Cho YK, Henning S, Harkins G. Broad ligament ectopic pregnancy after bilateral tubal ligation. Journal of Minimally Invasive Gynecology. 2018;25(2):314-315. Available from: https://linkinghub. elsevier.com/retrieve/pii/ S1553465017302212

[31] Phupong V, Lertkhachonsuk R, Triratanachat S, Sueblinvong T. Pregnancy in the broad ligament. Archives of Gynecology and Obstetrics. 2003;268(3):233-235. Available from: http://link.springer.com/10.1007/ s00404-002-0352-1

[32] Kobak AJ, Levine L. Interstitial pregnancy developing into the broad ligament. American Journal of Obstetrics and Gynecology. 1952;63(3):684, 6. Available from: http://www.ncbi.nlm.nih.gov/ pubmed/14902990

[33] Gupta A, Mahajan N, Verma D, Sharma C, Rattan A, Gupta B. Broad ligament pregnancy a diagnostic dilemma: A case report. International Journal of Reproduction, Contraception, Obstetrics and Gynecology. 2016: 2478-2480. Available from: http://www. ijrcog.org/index.php/ijrcog/article/ view/1388

[34] Ade-Ojo IP, Akintayo AA, Afolayan JM, Aduloju OP, OlagbujiBN.Intraligamentaryextrauterine pregnancy delivered at term: A case report and review of literature. African Journal of Reproductive Health. 2016;20(1):104-108. Available from: http://www.ncbi.nlm.nih.gov/ pubmed/29553183

[35] Sheethal CH, Powar A. Full term viable secondary broad ligament pregnancy-A rare case. Case reports women's Heal. 2017;13:4-5. Available from: http://www.ncbi.nlm.nih.gov/ pubmed/29593985

[36] Seckin B, Turkcapar FA, Tarhan I, Yalcin HR. Advanced intraligamentary pregnancy resulting in a live birth. Journal of Obstetrics and Gynaecology. 2011;31(3):260-261. Available from: http://www.ncbi.nlm.nih.gov/ pubmed/21417654

[37] Suchánková E, Pavlásek J, Bydžovská I, Lubušký M. Abdominal pregnancy at a patient after cesarean section, bilateral salpingectomy and embryotransfer. Ceskoslovenská Gynekologie;82(2):122-125. Available from: http://www.ncbi.nlm.nih.gov/ pubmed/28585844

[38] Siow A, Chern B, Soong Y. Successful laparoscopic treatment of an abdominal pregnancy in the broad ligament. Singapore Medical Journal. 2004;45(2):8889. Available from: http://www.ncbi.nlm. nih.gov/pubmed/14985849

[39] Cosentino F, Rossitto C, Turco LC, Gueli Alletti S, Vascone C, Di Meglio L, et al. Laparoscopic management of abdominal pregnancy.

Journal of Minimally Invasive Gynecology;24(5):724-725. Available from: http://www.ncbi.nlm.nih.gov/ pubmed/28179200

[40] Schramm M. Advanced intraligamentary pregnancy: A report of 2 cases. The Australian \& New Zealand Journal of Obstetrics \& Gynaecology. 1982;22(4):240-242. Available from: http://www.ncbi.nlm.nih.gov/ pubmed/6963166 
[41] Rakotomahenina $\mathrm{H}$,

Andrianampy HA, Ramamonjinirina P,

Solofomalala GD, Brun J-L. Post-term

pregnancy in the broad ligament.

Gynécologie, Obstétrique \&

Fertilité;42(7-8):537-539. Available

from: http://www.ncbi.nlm.nih.gov/

pubmed/24927650

[42] Dolinko AV, Vrees RA,

Frishman GN. Non-tubal ectopic

pregnancies: Overview and treatment

via local injection. Journal of Minimally

Invasive Gynecology. 2018;25(2):287-

296. Available from: https://

linkinghub.elsevier.com/retrieve/pii/

S1553465017303953

[43] Gracia C. Diagnosing ectopic pregnancy: Decision analysis comparing six strategies. Obstetrics and Gynecology. 2001;97(3):464-

470 Available from: http://

linkinghub.elsevier.com/retrieve/pii/ S0029784400011595

[44] Goldstein SR, Snyder JR, Watson C, Danon M. Very early pregnancy detection with endovaginal ultrasound. Obstetrics and Gynecology. 1988;72(2):200-204. Available from: http://www.ncbi.nlm.nih.gov/ pubmed/3292977

[45] Timor-Tritsch IE, Yeh MN, Peisner DB, Lesser KB, Slavik TA. The use of transvaginal ultrasonography in the diagnosis of ectopic pregnancy. American Journal of Obstetrics and Gynecology. 1989;161(1):157-161. Available from: http://www.ncbi.nlm. nih.gov/pubmed/2665494

[46] Agarwal N, Odejinmi F. Early abdominal ectopic pregnancy: Challenges, update and review of current management. The Obstetrician and Gynaecologist. 2014;16(3):193198. Available from: http://doi.wiley. com/10.1111/tog.12109

[47] Worley KC, Hnat MD, Cunningham FG. Advanced extrauterine pregnancy: Diagnostic and therapeutic challenges. American Journal of Obstetrics and Gynecology. 2008;198(3):297.e1-297.e7. Available from: http://www.ncbi.nlm.nih.gov/ pubmed/18313451

[48] Mausner Geffen E, Slywotzky C, Bennett G. Pitfalls and tips in the diagnosis of ectopic pregnancy. Abdominal Radiology. 2017;42(5):1524-1542 Available from: http://link.springer.com/10.1007/ s00261-016-1020-4

[49] Allibone GW, Fagan CJ, Porter SC. The sonographic features of intraabdominal pregnancy. Journal of Clinical Ultrasound. 1981;9(7):383-387. Available from: http://www.ncbi.nlm. nih.gov/pubmed/6792237

[50] Washington L, Thompson HE. Illustrated manual of ultrasonography in obstetrics and gynecology. Journal of Clinical Ultrasound. 1975;3(4):315316. Available from: http://doi.wiley. com/10.1002/jcu.1870030420

[51] Garrett WJ, Ward JP, Kossoff G, De Leon AH. Ultrasonic and radiological investigation of abdominal pregnancy. Australasian Radiology. 1975;19(4):334-337. Available from: http://www.ncbi.nlm.nih.gov/ pubmed/1225294

[52] Malian V, Lee JH. MR imaging and MR angiography of an abdominal pregnancy with placental infarction. American Journal of Roentgenology. 2001;177(6):1305-1306. Available from: http://www.ncbi.nlm.nih.gov/ pubmed/11717071

[53] Ghaneie A, Grajo JR, Derr C, Kumm TR. Unusual ectopic pregnancies. Journal of Ultrasound in Medicine. 2015;34(6):951-962. Available from: http://doi.wiley.com/10.7863/ ultra.34.6.951

[54] ACR Committee on Drugs and Contrast Media. ACR Manual On 
Contrast Media Version 10.3. 2018. pp. 95-98

[55] Radhakrishnan K. Radiological case: Intra-abdominal pregnancy. Applied Radiology. 2015:44-47

[56] Shamaash AH, Abbas AM. Undiagnosed asymptomatic second trimester broad ligament ectopic pregnancy: A case report and minireview. Proceedings in Obstetrics and Gynecology. 2016;7(1):1-8. Available from: http://ir.uiowa.edu/pog/vol7/iss1/8

[57] Practice Committee of American Society for Reproductive Medicine. Medical treatment of ectopic pregnancy: A committee opinion. Fertility and Sterility. 2013;100(3):638-644. Available from: http://www.ncbi.nlm.nih.gov/ pubmed/23849842

[58] Cachón López OR, Gasque López F, Peniche Rodríguez R, Avila Vergara MA. Abdominal pregnancy. Its conservative management. A case report with a live conceptus. Ginecología y Obstetricia de México. 1989;57:73-75. Available from: http://www.ncbi.nlm. nih.gov/pubmed/2487306

[59] Pisarska MD, Casson PR, Moise KJ, DiMaio DJ, Buster JE, Carson SA. Heterotopic abdominal pregnancy treated at laparoscopy. Fertility and Sterility. 1998;70(1):159160. Available from: http://www.ncbi. nlm.nih.gov/pubmed/9660440

[60] Sassi A, Dimassi K, Ben Slama S, Triki A, Lahmar A. A broad ligament pregnancy successfully managed by laparoscopy. Journal of Obstetrics and Gynaecology. 2018;38(3):423-424. Available from: http://www.ncbi.nlm. nih.gov/pubmed/28903610

[61] Mo X, Tang S, Zhou L-J-G-Y-K, Li C. Management of ectopic pregnancy in a broad ligament and recurrent tubal pregnancy: A case report. Open Journal of Obstetrics and Gynecology.
2018;08(05):431-436 Available from: http://www.scirp.org/journal/doi. aspx?DOI=10.4236/ojog.2018.85049

[62] Kar S. Primary abdominal pregnancy following intra-uterine insemination. The Journal of Human Reproductive Sciences. 2011;4(2):95-99. Available from: http://www.ncbi.nlm. nih.gov/pubmed/22064787

[63] Yang M, Cidan L, Zhang D. Retroperitoneal ectopic pregnancy: A case report and review of the literature. BMC Pregnancy and Childbirth. 2017;17(1):358. Available from: http://www.ncbi.nlm.nih.gov/ pubmed/29037192

[64] Mittal S, Chhabra P, Khanna R. Advanced abdominal intraligamentary pregnancy with live birth. International Journal of Gynaecology and Obstetrics. 1994;46(3):327-328. Available from: http://www.ncbi.nlm.nih.gov/ pubmed/7806005

[65] Paterson WG, Grant KA. Advanced intraligamentous pregnancy. Report of a case, review of the literature and a discussion of the biological implications. Obstetrical \& Gynecological Survey. 1975;30(11):715-726. Available from: http://www.ncbi.nlm.nih.gov/ pubmed/1187075

[66] Mittal S, Gupta V, Chawla D, Pundir S. Broad ligament ectopic pregnancy: A dilemma to diagnose. International Journal of Reproduction, Contraception, Obstetrics and Gynecology. 2017;6(5):2109. Available from: http://www.ijrcog.org/index.php/ ijrcog/article/view/2566

[67] Naeiji Z, Saleh M, Keshavarz E. Broad ligament pregnancy: A case report. International Journal of Reproduction, Contraception, Obstetrics and Gynecology. 2015;9:118-122

[68] Singh U, Singh N, Sankhwar P. Fullterm viable broad ligament pregnancy 
Broad Ligament Pregnancy

DOI: http://dx.doi.org/10.5772/intechopen.88986

surgically managed with favorable feto-maternal outcome. Journal of Obstetrics and Gynaecology of India. 2012;62 (Suppl 1):23-24. Available from: http://www.ncbi.nlm.nih.gov/ pubmed/24293863 


\section{Edited by Julio Elito Jr.}

"Non-Tubal Ectopic Pregnancy" is a comprehensive book, written in an organized and concise format. The book offers an immersion into non-tubal ectopic pregnancy and the reader is invited, chapter after chapter, to visit the most important aspects of nontubal ectopic pregnancies.

The book covers all aspects of non-tubal ectopic pregnancies including epidemiology, diagnosis, and management. Experts from all over the world have contributed to it, bringing the best from their research.The book presents the reader with the latest advances on non-tubal ectopic pregnancies. 